\title{
Design, Synthesis, In Silico and In Vitro Studies for New Nitric Oxide-Releasing Indomethacin Derivatives with 1,3,4-Oxadiazole-2-thiol Scaffold
}

\author{
Alexandru Sava ${ }^{1,2}\left(\mathbb{D}\right.$, Frederic Buron ${ }^{2}\left(\mathbb{D}\right.$, Sylvain Routier ${ }^{2, *},{ }^{+}$, Alina Panainte ${ }^{1}$, Nela Bibire ${ }^{1}$, \\ Sandra Mădălina Constantin ${ }^{3}$, Florentina Geanina Lupașcu ${ }^{3}$, Alin Viorel Focșa ${ }^{3}$ and Lenuţa Profire ${ }^{3, *},+(\mathbb{D}$ \\ 1 Department of Analytical Chemistry, Faculty of Pharmacy, “Grigore T. Popa” University of Medicine and \\ Pharmacy of Iași, 16 University Street, 700115 Iasi, Romania; alexandru.i.sava@umfiasi.ro (A.S.); \\ alina-diana.panainte@umfiasi.ro (A.P.); nela.bibire@umfiasi.ro (N.B.) \\ 2 Institut de Chimie Organique et Analytique ICOA, CNRS UMR 7311, Université d'Orléans, \\ 45067 Orléans, France; frederic.buron@univ-orleans.fr \\ 3 Department of Pharmaceutical Chemistry, Faculty of Pharmacy, "Grigore T. Popa" University of Medicine \\ and Pharmacy of Iași, 16 University Street, 700115 Iasi, Romania; constantin.sandra@umfiasi.ro (S.M.C.); \\ florentina-geanina.lupascu@umfiasi.ro (F.G.L.); alin-viorel-v-focsa@d.umfiasi.ro (A.V.F.) \\ * Correspondence: sylvain.routier@univ-orleans.fr (S.R.); lenuta.profire@umfiasi.ro (L.P.) \\ + Both authors contributed equally as senior authors.
}

Citation: Sava, A.; Buron, F.; Routier, S.; Panainte, A.; Bibire, N.; Constantin, S.M.; Lupașcu, F.G.; Focșa, A.V.; Profire, L. Design, Synthesis, In Silico and In Vitro Studies for New Nitric Oxide-Releasing Indomethacin Derivatives with 1,3,4-Oxadiazole-2-thiol Scaffold. Int. J. Mol. Sci. 2021, 22, 7079. https:// doi.org/10.3390/ijms22137079

Academic Editor: Antonio

Palumbo Piccionello

Received: 25 May 2021

Accepted: 25 June 2021

Published: 30 June 2021

Publisher's Note: MDPI stays neutral with regard to jurisdictional claims in published maps and institutional affiliations.

Copyright: (c) 2021 by the authors. Licensee MDPI, Basel, Switzerland. This article is an open access article distributed under the terms and conditions of the Creative Commons Attribution (CC BY) license (https:// creativecommons.org/licenses/by/ $4.0 /)$.

\begin{abstract}
Starting from indomethacin (IND), one of the most prescribed non-steroidal anti-inflammatory drugs (NSAIDs), new nitric oxide-releasing indomethacin derivatives with 1,3,4-oxadiazole-2-thiol scaffold (NO-IND-OXDs, 8a-p) have been developed as a safer and more efficient multitarget therapeutic strategy. The successful synthesis of designed compounds (intermediaries and finals) was proved by complete spectroscopic analyses. In order to study the in silico interaction of NO-IND-OXDs with cyclooxygenase isoenzymes, a molecular docking study, using AutoDock 4.2.6 software, was performed. Moreover, their biological characterization, based on in vitro assays, in terms of thermal denaturation of serum proteins, antioxidant effects and the NO releasing capacity, was also performed. Based on docking results, $\mathbf{8 k}, \mathbf{8 1}$ and $\mathbf{8 m}$ proved to be the best interaction for the COX-2 (cyclooxygense-2) target site, with an improved docking score compared with celecoxib. Referring to the thermal denaturation of serum proteins and antioxidant effects, all the tested compounds were more active than IND and aspirin, used as references. In addition, the compounds $8 \mathrm{c}, \mathbf{8 h}, \mathbf{8 i}, \mathbf{8 m}, \mathbf{8 n}$ and 80 showed increased capacity to release $\mathrm{NO}$, which means they are safer in terms of gastrointestinal side effects.
\end{abstract}

Keywords: indomethacin; 1,3,4-oxadiazole-2-thiol; nitric oxide; docking study; cyclooxygenase; inflammation

\section{Introduction}

The non-steroidal anti-inflammatory drugs (NSAIDs) are the most prescribed drugs for management of different pathological conditions where inflammation is involved, based on their analgesic, anti-inflammatory and antipyretic effects [1,2].

The inflammation is a defense reaction of the human body to various harmful agents in restoring the body homeostasis [3-6]. When inflammation persists for a long time, holding the body in a constant state of alert, it may become chronic, with a negative impact on tissue and organs [7]. The clinical consequences of chronic inflammation-driven damage can be severe and include increased risk of many chronic diseases such as: rheumatoid arthritis [8], inflammatory bowel disease [9], metabolic disorders (diabetes mellitus and obesity) [10-12], cardiovascular disorders (ischemic heart disease, atherosclerosis) [13,14], neurodegenerative diseases (Parkinson's and Alzheimer's disease) [15-17] and cancer [18], many of these conditions being life-threatening $[19,20]$. The current use of NSAIDs in the European Union is associated with a $19 \%$ increased risk of hospital admissions for heart 
failure compared to periods before 1999-2012, as is well documented [21-23]. Despite the clinical benefits, the chronic use of NSAIDs is also associated with increased risk of side effects, including gastrointestinal toxicity, renal injury, hepatotoxicity, hypertension, as well as allergic skin reactions [24-27]. The main mechanism of NSAIDs' action, which is responsible for both therapeutic and side effects, is inhibition of cyclooxygenase (COX) enzymes in a wide variety of systems, ranging from microsomal enzyme synthesis to different cells and tissues $[24,28,29]$. Moreover, in the last two decades, based on the beneficial effects of endogenous nitric oxide (NO), NSAIDs with NO-releasing moiety were developed as a new therapeutic strategy for a variety of clinical conditions which involve acute and chronic inflammation [30-34]. Nitric oxide (NO), an endogenous short-lived free radical, is produced in mammalian cells through nitric oxide synthase-mediated conversion of L-arginine to L-citroline [35-38]. This important signaling molecule has a key role in a wide variety of biological processes such as immune defenses, inflammation, neurotransmission, vasodilatation, platelet adhesion, thrombosis and wound healing [39-41]. In addition, NO is intimately involved in regulating all aspects of our lives from waking, digestion, sexual function, perception of pain and pleasure, memory recall and sleeping [40,41]. Moreover, $\mathrm{NO}$ is known to have a protective effect on the gastrointestinal tract (GT), based on its properties in stimulating gastric mucus secretion to increase the mucosal blood flow and to inhibit the leukocyte adherence to the vascular endothelium $[39,42,43]$.

The design of hybrid molecules, able to reduce the inflammation by both COX inhibition and NO release could be of great interest. The aim of our study was to develop new nitric oxide-releasing indomethacin derivatives with 1,3,4-oxadiazole-2-thiol scaffold (NO-IND-OXDs), as a safer and more efficient multitarget therapeutic strategy. Among the library of COX inhibitors, indomethacin (IND) retains our attention as it is one of the most prescribed NSAIDs in the management of osteoarthritis, rheumatoid arthritis, episodes of acute gout, ankylosing spondylitis and acute musculoskeletal pain [44,45]. The long-term use of IND could increase the risk for a wide range of side effects such as gastrointestinal irritation, bleeding and ulceration, dizziness, peripheral edema, arterial hypertension, tachycardia, kidney and liver dysfunction, allergic and anaphylactic reactions, increased anxiety, headache $[44,46]$.

On the other hand, the oxadiazole scaffold serves as core for many synthetic compounds of great interest in medicinal chemistry [47-49]. Its use offers several advantages: (i) it is an essential part of the pharmacophore, based on its ligand binding role; (ii) it acts as a flat aromatic linker assuring an appropriate molecule orientation; (iii) it induces metabolic stability, water solubility and lower lipophilicity; (iv) it can easily chemically modulate the compounds which contain carbonyl groups such as amides, carbamates, esters and hydoxamic esters [32,50,51]. According to the literature data, the compounds containing the oxadiazole core, generally 1,3,4-oxadiazole motif, have important biological effects such as anti-inflammatory [32,52,53], antioxidant [54,55], antidiabetic [56], anticonvulsant [57], anticancer [58], antitubercular [59,60], antiviral [61], antidepressant [62]. There have already been several approved oxadiazole-based drugs such as: furamizole (strong antibacterial activity, an antibiotic), butalamine (a vasodilator), oxolamine (a cough suppressant), pleconaril (an antiviral), fasiplon (a non-benzodiazepine anxiolitic drug), raltegravir (an antiretroviral drug for the treatment of HIV infection), nesapidil (an anti-arrhythmic drug), zibotentan (an anticancer drug) as well as tiodazosin (an antihypertensive drug) (Figure 1).

In nature, the oxadiazole scaffold has been reported to occur in quisqualic acid (from Quiqualis fructus, strong agonist of (AMPA)-subtype glutamate receptor) and phidianidines $\mathrm{A}$ and $\mathrm{B}$ (from primitive marine organisms, high cytotoxicity against tumor mammalian cell lines) $[63,64]$.

Moreover, the designed compounds contain, between NO precursor and oxadiazole unit, an aromatic linker, substituted with different electron-withdrawing and electrondonating groups. This fragment may serve as a decoy that promotes the binding of the COX to the designed NO-IND-OXDs, according to the reported substrate trap strategies [65-68]. 


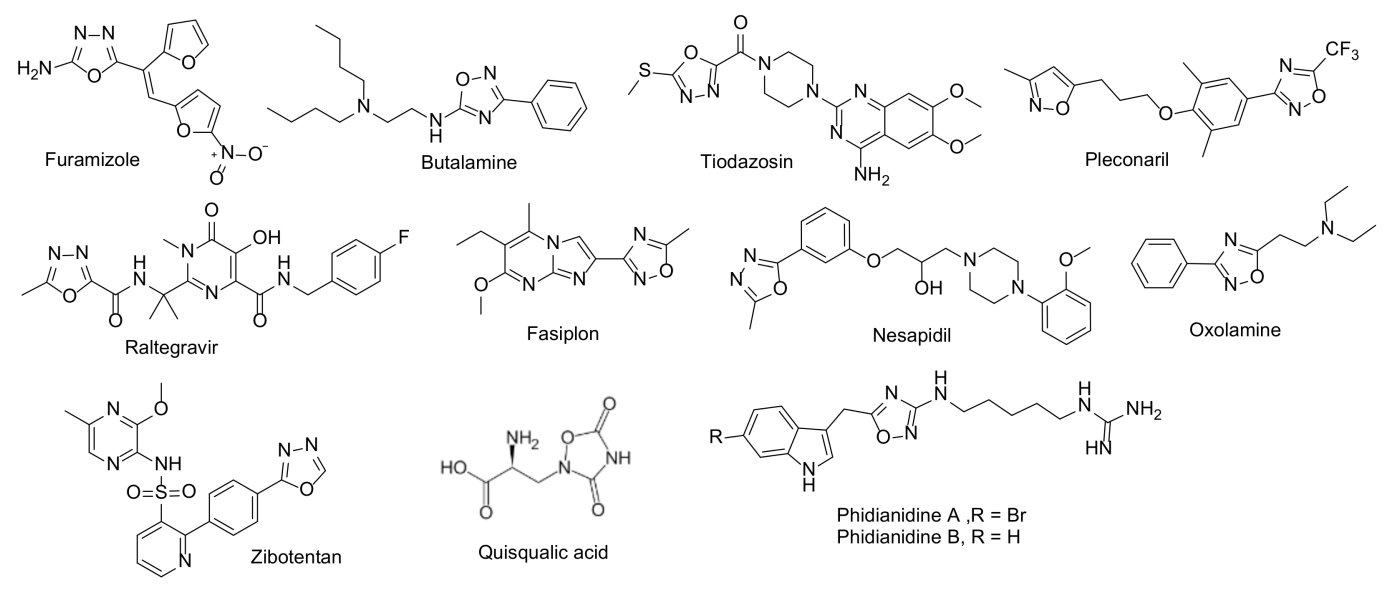

Figure 1. Representative drugs and natural products containing the oxadiazole core.

Based on presented aspects, the chemical modulation of the free carboxyl group of conventional NSAIDs, such as indomethacin, with 1,3,4-oxadiazole scaffold, can provide new drugs with an improved pharmacological profile, in terms of increased efficiency, fewer side effects and reduced ulcerogenic potential. Moreover, the presence of two pharmacophors (indol and 1,3,4-oxadiazole moieties) could increase the ability to inhibit COX enzymes, especially COX-2.

Herein, we present the design and synthesis of some new NO-IND-OXDs, in silico interaction with COX isoenzymes, based on a molecular docking study, as well as the biological characterization using in vitro assays in terms of thermal denaturation of serum proteins, antioxidant effects and the NO-releasing capacity.

\section{Results and Discussion}

\subsection{Chemistry}

Based on reactivity of the carboxyl group of IND, new NO-IND-OXDs 8a-p were prepared in a few steps (Scheme 1). Reaction of hydroxy-benzaldehydes 1a-o and 4-(2hydroxyethyl)phenol $\mathbf{2 p}$ with 1,2-dibromoethane, afforded on an $\mathrm{SN}_{2}$ Williamson ether synthesis, gave the corresponding (bromoethoxy)benzaldehyde derivatives $\mathbf{2 a - 0}$ and 2(4-(2-bromoethoxy)phenyl)ethan-1-ol 3p, respectively. In the next step, the intermediaries 2a-o were reduced by standard procedure with $\mathrm{NaBH}_{4}$ into their corresponding (bromoethoxy)aromatic primary alcohols 3a-o in a good yield (86-99\%). This method offered significant advantages such as mild reaction conditions and easy isolation (simple neutralization and extraction) of the product.

By the metathesis reaction of $\mathbf{3 a - p}$ with silver nitrate in acetonitrile, the corresponding (hydroxyalkyl)phenoxy nitrates $\mathbf{4 a - p}$ were obtained, which were further reacted with thionyl chloride and iodine, respectively, to form the corresponding (halidealkyl)phenoxy nitrates $5 \mathbf{a}-\mathbf{p}$. In turn, the IND derivative $\mathbf{7}$ was obtained by peptide coupling reaction between IND and hydrazine hydrate when the IND hydrazide 6 was obtained, which further was reacted with carbon disulfide in presence of triethylamine leading to (4-chlorobenzoyl)5-methoxy-2-methyl-1H-indol-3-yl)methyl)-1,3,4-oxadiazol 7. Finally, the intermediates $\mathbf{5 a}-\mathbf{p}$ were used to alkylate the IND derivative 7 in presence of triethylamine to form the desired NO-IND-OXDs 8a-p. The common methods for conversion of alcohols to the corresponding alkyl chloride involve activation of hydroxyl group before treatment with the chlorination agent [69]. In the present study we used thionyl chloride in combination with benzotryazole as the activation agent. Benzotryazole is a mild base as well as acid and offers several advantages. It can be removed easily from the reaction mixture by acid or alkali and its hydrochloride salt is insoluble in organic solvents such as dichloromethane [70,71]. Using this method, the desired compounds $\mathbf{5 a}-\mathbf{m}, \mathbf{t}$, $\mathbf{u}$ were obtained at room temperature in high yields which ranged between 85 and $99 \%$. 

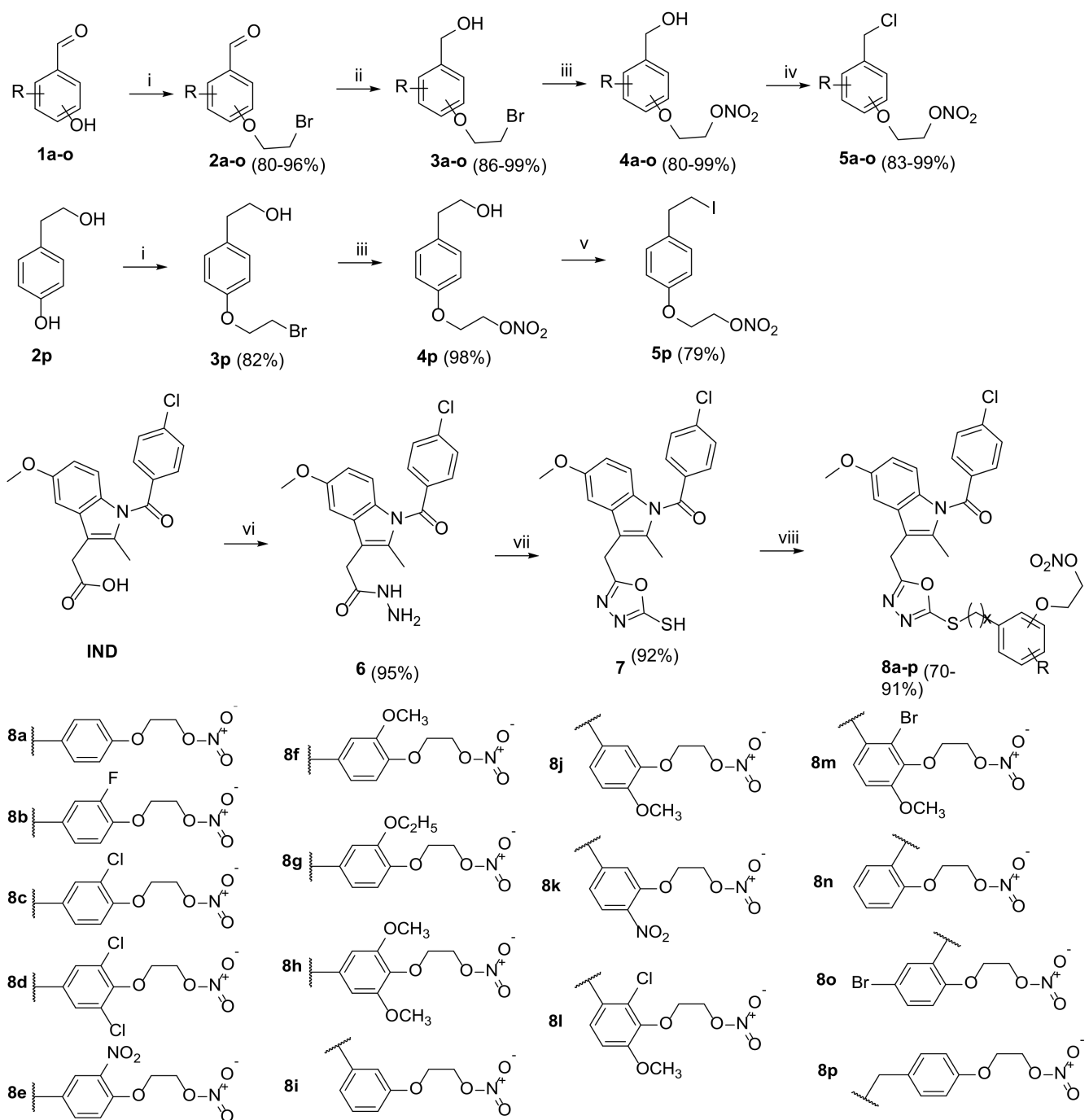

Scheme 1. Synthesis of NO-IND-OXDs 8a-p. Reagents and conditions: (i) $\mathrm{C}_{2} \mathrm{H}_{4} \mathrm{Br}_{2}, \mathrm{~K}_{2} \mathrm{CO}_{3} / \mathrm{CH}_{3} \mathrm{CN}$, $95^{\circ} \mathrm{C}$, 18-26 h, (ii) $\mathrm{NaBH}_{4} / \mathrm{THF}$, RT, $12-24 \mathrm{~h}$, (iii) $\mathrm{AgNO}_{3} / \mathrm{CH}_{3} \mathrm{CN}$, $95^{\circ} \mathrm{C}, 8-10 \mathrm{~h}$, (iv) $\mathrm{SOCl}_{2}$, BTA/dry $\mathrm{CH}_{2} \mathrm{Cl}_{2}$, RT, 20-30 min, (v) imidazole, $\mathrm{PPh}_{3}, \mathrm{I}_{2} / \mathrm{CH}_{2} \mathrm{Cl}_{2}, \mathrm{RT}, 6 \mathrm{~h}$, (vi) $\mathrm{HOBt}, \mathrm{EDC} / \mathrm{CH}_{3} \mathrm{CN}$, RT, 3h, $\mathrm{N}_{2} \mathrm{H}_{4}\left(0-5{ }^{\circ} \mathrm{C}, 1 \mathrm{~h}\right.$ ), (vii) $\mathrm{Et}_{3} \mathrm{~N}, \mathrm{CS}_{2} / \mathrm{CH}_{3} \mathrm{CN}$, reflux, $3 \mathrm{~h}$, (viii) 5a-p, Et ${ }_{3} \mathrm{~N} / \mathrm{CH}_{3} \mathrm{CN}, \mathrm{RT}, 3-6 \mathrm{~h}$.

The compound 7 was obtained in near quantitative yield (92\%) and was somewhat easier (reaction time of $3 \mathrm{~h}$ ) while NO-IND-OXDs were obtained at room temperature in good yields ranged between 70 and $91 \%$.

The chemical structure of all synthesized compounds (intermediate and final compounds) was proven on the basis of nuclear magnetic resonance $\left({ }^{1} \mathrm{H} \mathrm{NMR},{ }^{13} \mathrm{C} \mathrm{NMR}\right)$ and high-resolution mass spectrometry (HRMS) analysis (Supplementary Materials).

In the ${ }^{1} \mathrm{H}$ NMR spectra of (halidealkyl)phenoxy nitrates $5 a-p$ it was found that the multiple signal of protons $\left(-\mathrm{CH}_{2} \mathrm{ONO}_{2}\right)$ that are in the vicinity of electron-withdrawing nitrate ester group appear more unshielded $(\delta=4.7-4.9 \mathrm{ppm}, \mathrm{m}, 2 \mathrm{H})$, compared with the protons in the vicinity of bromide $\left(-\mathrm{CH}_{2} \mathrm{Br}\right)(\delta=3.7-3.90 \mathrm{ppm}, \mathrm{m}, 2 \mathrm{H})$ from (bromoethoxy)aromatic alcohol derivatives 3a-p. At the same time, ${ }^{13} \mathrm{C}$ NMR signal for $-\mathrm{CH}_{2} \mathrm{ONO}_{2}$ was observed at $\delta=72-73 \mathrm{ppm}$, compared to the signal of the same carbon from derivatives 3a-p $(\delta=31-32 \mathrm{ppm})$. In ${ }^{1} \mathrm{H}$ NMR spectra, the final NO-IND-OXDs $8 \mathbf{a}-\mathbf{p}$ showed signals for $\mathrm{CH}_{2} \mathrm{ONO}_{2}$ and $\mathrm{CH}_{2} \mathrm{~S}$ in the range of $\delta=4.7-4.9 \mathrm{ppm}$, and $\delta=4.3-4.5 \mathrm{ppm}$, respectively. Whereas in ${ }^{13} \mathrm{C}$ NMR spectra the signal for $\mathrm{CH}_{2} \mathrm{ONO}_{2}$ group was observed in the range of $\delta=71-72 \mathrm{ppm}$, the signal for $\mathrm{CH}_{2} \mathrm{~S}$ was observed in the range of $\delta=34-36 \mathrm{ppm}$ and for quaternary carbon from second position of 1,3,4-oxadiazol scaffold $\left(\mathrm{C}_{\mathrm{q}} \mathrm{S}\right)$ in the range of $\delta=162-163 \mathrm{ppm}$. The proton and carbon signals of other aliphatic and aromatic frag- 
ments of 8a-p were observed at the expected values of chemical shift. The presence of the abovementioned signals have confirmed the presence of 2-mercapto-1,3,4-oxadiazole scaffold and the preserved nitrate ester moiety in the final compounds. Moreover, the NMR spectral data coupled with mass spectra support the proposed structures of the all-synthesized compounds.

\subsection{In Silico Docking Study}

To predict the binding mode of the synthesized compounds with the COX isoenzymes, two X-ray crystallographic structures, obtained from crystallographic data, were used [72]. The PDB 4o1z and PDB 3nt1 were described as the COX-1 and COX-2 structures with the highest resolution to date, $2.4 \AA$ and $1.7 \AA$, respectively [73], which means that there is more confidence in the location of atoms in the electron density map. Furthermore, the reliability factor was less than 0.16 , indicating a strong agreement between the simulated and the experimentally observed diffraction patterns [74,75].

The used computational method was validated using RSMD variation at less than $2 \AA$ for reference drug (indomethacin-IND, diclofenac-DCF and celecoxib-CCB, individually) into the active site of both COX-1 and COX- 2 isoforms and the same binding cleft residues reported by other authors were found [76-79]. The CCB is a preferential COX-2 selective inhibitor whereas IND and DCF are non-selective COX inhibitors, inhibiting both types of the COX enzymes.

Moreover, the docking results showed that all reference drugs have polar interactions with the catalytic site of both COX-1 and COX-2 isoenzymes. More specifically, they act as hydrogen bond acceptors with Arg120 and Tyr355, and have long-range polar interactions with Tyr385 and Ser530 and non-polar interactions with Val349, Trp387, Phe-518, Ile/Val523 and Ala527 [80,81].

For estimating the docking score of NO-IND-OXDs 8a-p each ligand-receptor complex was subjected to careful analysis for the ideal docked positions, based on the least binding energy scores and maximum number of cluster conformations.

In order to establish the statistical significance of the difference between the ligands while removing the receptor variances from the overall error variance term, a two-way ANOVA test was applied. The results established a statistically significant difference between ligands $(F(0.5,18,37)=3.2648, p=0.0079$, Fcrit $=2.2171)$. The value of the estimated free binding energy of the compounds $8 \mathbf{a}-\mathbf{p}$ was less than $-10 \mathrm{kcal} / \mathrm{mol}$ and less than that of the reference drugs (IND, DCF and CCB) (Table 1). Therefore, less energy is needed to stabilize the synthesized compounds $\mathbf{8 a - p}$ at the ligand-binding center of the COX receptor.

Based on inhibitory constant (Ki) for COX-1 and COX-2 [82], a selectivity index $\left(\log _{10} \mathrm{Ki}_{\mathrm{COX}-1} / \mathrm{Ki}_{\mathrm{COX}-2}\right)$ was also calculated for docked NO-IND-OXDs $8 \mathbf{a}-\mathbf{p}$ (Table 1 and Figure 2). It was noted that the studied compounds have a better interaction with the COX-2 target site, with improved selective index in reference to IND and DCF, except $\mathbf{8 n}$, $\mathbf{8 f}$ and $\mathbf{8 h}$, which have a better docking score for COX-1 target site. Even more, it was observed that $8 \mathrm{~m}, \mathbf{8 1}$ and $\mathbf{8 k}$ proved to be the best interaction COX-2 target sites in reference with CCB (Figure 2).

The COX binding pocket, for both COX-1 and COX-2, is a hydrophobic channel which comprises four $\alpha$-helices, thus creating a hydrophobic surface to the core of the catalytic domain. In the upper part of the channel, both isoenzymes have two important amino acids, Ser530 and Tyr385. Ser530 influences the COX stereochemistry in prostaglandin synthesis while Tyr385 is involved in the hydroperoxidase activity. Moreover, towards the bottom of the COX active site there are two polar amino acids, Arg120 and Tyr355, which together form a narrow constriction at the entrance of the channel.

The main difference between the active sites of COX isoenzymes consists in the replacement of Ile (Ile434 and Ile523) in COX-1 by the less bulky amino acid Val (Val434 and Val523) in COX-2. A single methylene group (Ile vs. Val) is enough to open a secondary 
internal hydrophobic side pocket in COX-2 that enlarges the volume of the active site by approximately $25 \%$ and gives access to Arg513 replaced in COX-1 by a His.

Table 1. Docking score $(\Delta \mathrm{G}, \mathrm{kcal} / \mathrm{mol})$ and inhibition effect rate $(\mathrm{Ki})$ during docking simulation of NO-IND-OXDs 8a-p and reference drugs (IND, DCF, CCB) for COX-1 and COX-2.

\begin{tabular}{|c|c|c|c|c|c|}
\hline \multirow{2}{*}{ Ligand } & \multicolumn{2}{|c|}{ COX-1 } & \multicolumn{2}{|c|}{ COX-2 } & \multirow{2}{*}{$\begin{array}{c}\text { Selectivity Index } \\
\log _{10}\left(\mathrm{Ki}_{\text {COX-1 }} / \mathrm{Ki}_{\text {COX-2 }}\right)\end{array}$} \\
\hline & $\Delta \mathrm{G}(\mathrm{kcal} / \mathrm{mol})$ & $\mathrm{Ki}(\mathrm{nM})$ & $\Delta \mathrm{G}(\mathrm{kcal} / \mathrm{mol})$ & $\mathrm{Ki}(\mathrm{nM})$ & \\
\hline $8 a$ & -12.68 & 0.508 & -13.18 & 0.219 & 0.364 \\
\hline $8 b$ & -11.52 & 3.590 & -12.32 & 0.925 & 0.589 \\
\hline $8 c$ & -11.15 & 6.710 & -13.14 & 0.232 & 1.461 \\
\hline $8 d$ & -12.37 & 0.860 & -13.45 & 0.137 & 0.796 \\
\hline $8 e$ & -11.50 & 3.700 & -12.67 & 0.512 & 0.859 \\
\hline $8 f$ & -11.91 & 1.860 & -10.18 & 34.800 & -1.272 \\
\hline $8 g$ & -12.27 & 1.010 & -13.84 & 0.072 & 1.146 \\
\hline $8 \mathrm{~h}$ & -12.60 & 0.584 & -11.66 & 2.840 & -0.687 \\
\hline $8 \mathrm{i}$ & -12.76 & 0.442 & -13.37 & 0.157 & 0.449 \\
\hline $8 \mathbf{j}$ & -12.03 & 1.520 & -12.79 & 0.419 & 0.559 \\
\hline $8 k$ & -11.03 & 8.240 & -13.51 & 0.124 & 1.821 \\
\hline 81 & -10.75 & 13.140 & -13.2 & 0.209 & 1.796 \\
\hline $8 m$ & -11.42 & 4.270 & -13.58 & 0.110 & 1.587 \\
\hline $8 n$ & -11.85 & 2.050 & -7.88 & 1680 & -2.914 \\
\hline 80 & -12.55 & 0.631 & -13.2 & 0.211 & 0.476 \\
\hline $8 p$ & -12.72 & 0.470 & -14.27 & 0.034 & 1.129 \\
\hline IND & -9.98 & 48.230 & -10.35 & 25.720 & 0.273 \\
\hline DCF & -8.12 & 1120 & --8.63 & 468.540 & 0.378 \\
\hline CCB & -8.37 & 737.590 & -10.31 & 27.520 & 1.428 \\
\hline
\end{tabular}

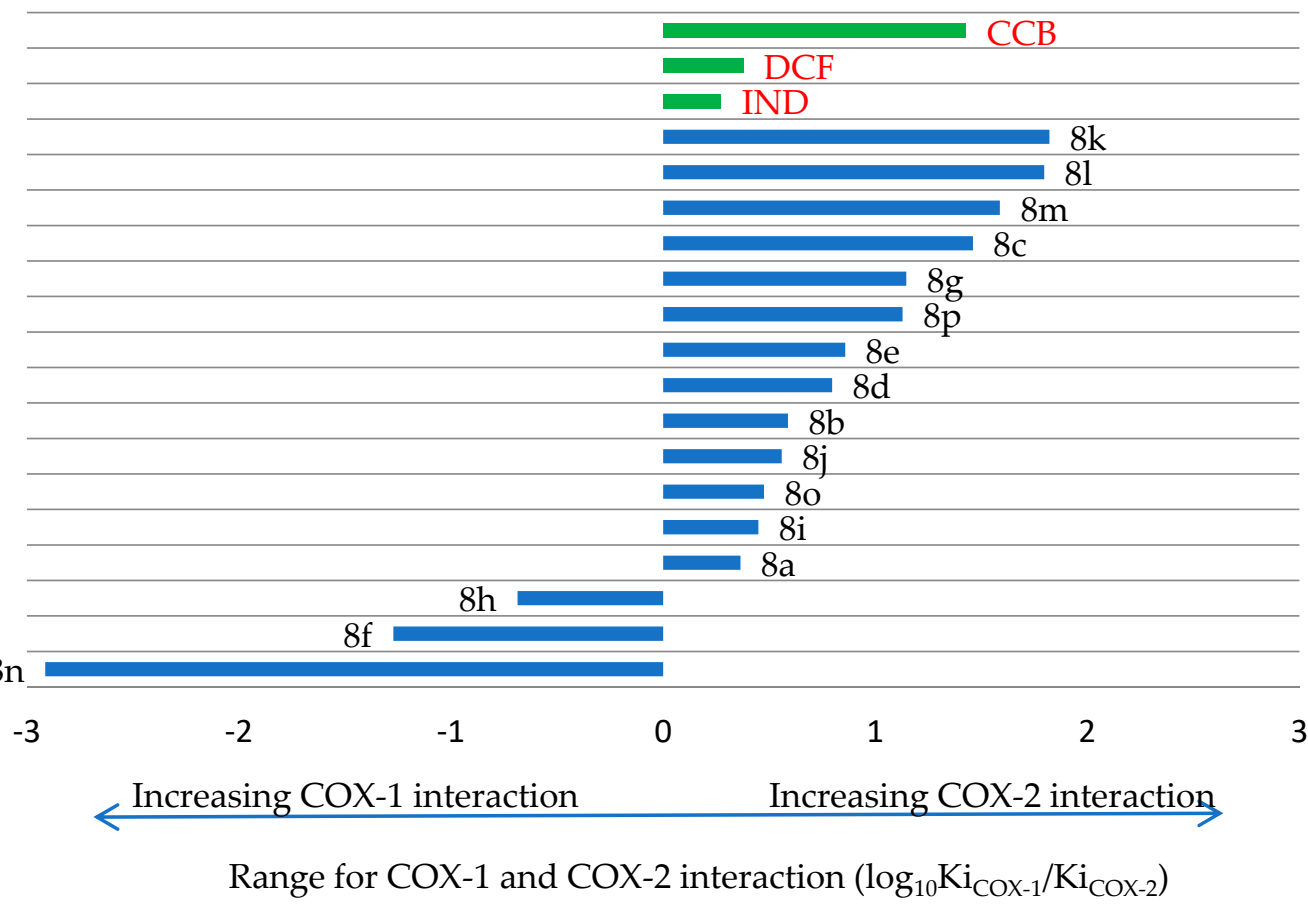

Figure 2. The COX interaction of NO-IND-OXDs $8 \mathbf{a}-\mathbf{p}$ in respect to reference drugs.

Referring to the binding of NO-IND-OXDs $\mathbf{8 a}-\mathbf{p}$ to COX-1, differences were noted between compounds. However, it was observed that all compounds interact with amino acids both from the narrow constriction and the inside of COX-1 active site (Figure 3a). The binding mode of NO-IND-OXDs $\mathbf{8} \mathbf{a}-\mathbf{p}$ to COX-2 side pocket is quite similar (Figure 3b) for all compounds, as they have affinity for the hydrophobic channel. It was noted that the 
NO-releasing chain interacts with the site pocket (delimited by Val523, Phe518, Arg513, Ala516, Gln192, Tyr355) and extra space (delimited by Leu384, Leu503, Tyr385, Trp387). The oxadiazole scaffold interacts by hydrogen bonds with Arg120 and Tyr355 from the narrow constriction while the indole structure interacts with amino acids from the entrance of the active site.

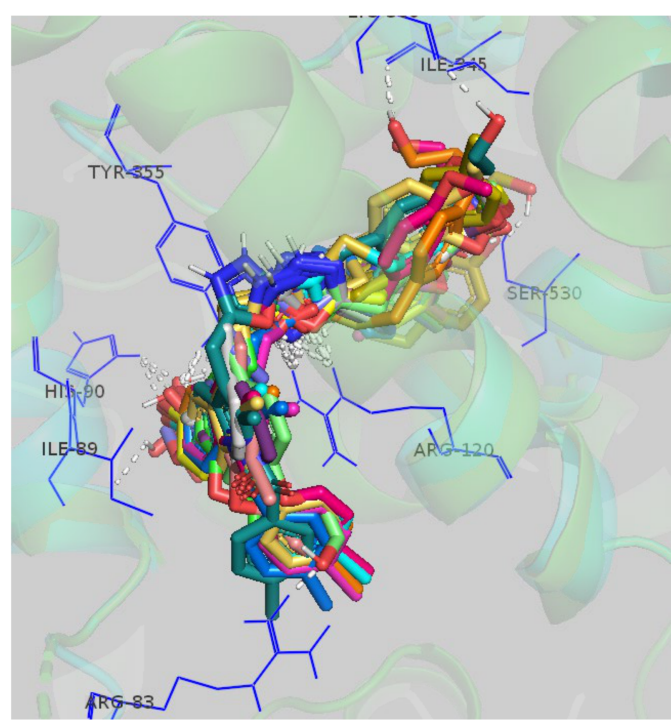

(a)

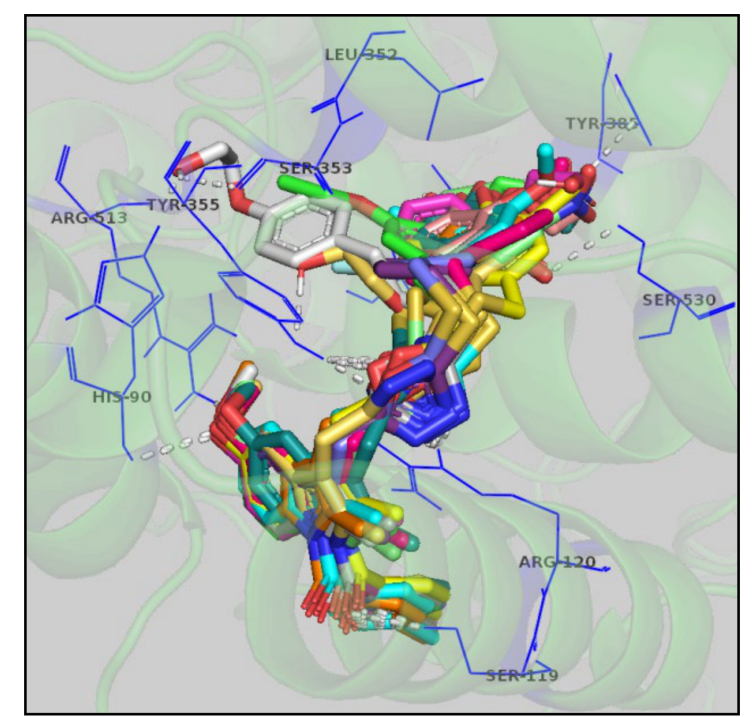

(b)

Figure 3. Binding mode of $\mathbf{8 a - p}$ to the active site of COX-1 (a) and COX-2 (b).

The analysis of the results revealed that the COX-1/COX-2 selectivity of $\mathbf{8 a}-\mathbf{p}$ is influenced by the position of nitrate ester moiety on aromatic ring and by the number and type of the substituents on aromatic ring. The most proper positions of nitrate ester moiety are meta and para, $\mathbf{8 i}$ (3-oxy-ethylnitrate) and 8a (4-oxy-ethylnitrate), respectively, being more active on COX-2 than $8 \mathbf{n}$ (2-oxy-ethylnitrate), which is more COX-1-selective. Referring to the substitution of phenoxy-ethylnitrate moiety, the presence of halogens $(\mathrm{F}$, $\mathrm{Cl}, \mathrm{Br}$ ) or $\mathrm{NO}_{2}$ on aromatic ring increases the COX-2 selectivity index, in contrast with only $\mathrm{OCH}_{3}(\mathbf{8 h}, \mathbf{8 f})$, which increases COX-1 selectivity. Moreover, it was observed that the elongation of distance between oxadiazole moiety and aromatic ring, from methylene (8a) to ethylene (8p), seems to be responsible for better ligand flexibility and selectivity for the COX-2 active site. Based on the obtained results, we can say that the synthesized NO-INDOXDs have the theoretical premises to be promising anti-inflammatory agents. This is in agreement with the literature which reported that the replacement of the free carboxylic acid group of conventional NSAID with 1,3,4-oxadiazole heterocycle may provide new drugs with increased anti-inflammatory activity, improved efficiency and with fewer side effects, reducing ulerogenic potential $[53,83]$.

\subsection{In Vitro Radical Scavenging Assay}

The most used radical scavenging assay is based on the reduction of the DPPH $(2,2-$ diphenyl-1-picrylhydrazyl radical), in the presence of a proton- or electron-donating agent, when there is a color changing from violet to yellow [79]. In order to evaluate the scavenging effect of NO-IND-OXDs, the concentration needed to decrease the initial DPPH concentration $\left(\mathrm{IC}_{50}\right.$ ) by $50 \%$ was calculated and the results are presented in Table 2 . Furthermore, the vitamin C equivalent antioxidant capacity (CEAC) value was also calculated, in order to compare the antioxidant effect of $\mathbf{8 a - p}$ to vitamin $\mathbf{C}$ (Figure 4). 
Table 2. The $\mathrm{IC}_{50}$ and CEAC values of NO-IND-OXDs $8 \mathbf{a}-\mathbf{p}$ referring to the DPPH radical scavenging effect.

\begin{tabular}{cccccc}
\hline Comp. & IC $_{\mathbf{5 0}}(\mathbf{m M})^{\mathbf{a}}$ & CEAC $^{\mathbf{a}}$ & Comp. & IC $_{\mathbf{5 0}}(\mathbf{m M})^{\mathbf{a}}$ & CEAC $^{\mathbf{a}}$ \\
\hline $\mathbf{8 a}$ & $2.38 \pm 0.02$ & $0.0309 \pm 0.0002$ & $\mathbf{8 k}$ & $2.19 \pm 0.01$ & $0.0336 \pm 0.0001$ \\
$\mathbf{8 b}$ & $4.35 \pm 0.34$ & $0.0170 \pm 0.0012$ & $\mathbf{8 1}$ & $5.29 \pm 0.21$ & $0.0139 \pm 0.0005$ \\
$\mathbf{8} \mathbf{c}$ & $2.37 \pm 0.01$ & $0.0311 \pm 0.0001$ & $\mathbf{8 m}$ & $2.9 \pm 0.31$ & $0.0256 \pm 0.0029$ \\
$\mathbf{8 d}$ & $2.46 \pm 0.02$ & $0.0299 \pm 0.0003$ & $\mathbf{8 n}$ & $2.85 \pm 0.06$ & $0.0258 \pm 0.0005$ \\
$\mathbf{8} \mathbf{e}$ & $2.16 \pm 0.03$ & $0.0341 \pm 0.0004$ & $\mathbf{8 0}$ & $4.03 \pm 1.11$ & $0.0192 \pm 0.0047$ \\
$\mathbf{8 f}$ & $2.42 \pm 0.02$ & $0.0305 \pm 0.0003$ & $\mathbf{8 p}$ & $2.77 \pm 0.03$ & $0.0265 \pm 0.0003$ \\
$\mathbf{8 g}$ & $2.98 \pm 0.06$ & $0.0248 \pm 0.0005$ & $\mathbf{A S P}$ & $4.58 \pm 0.10$ & $0.0162 \pm 0.0004$ \\
$\mathbf{8 h}$ & $2.49 \pm 0.02$ & $0.0296 \pm 0.0003$ & $\mathbf{I N D}$ & $54.37 \pm 7.94$ & $0.0014 \pm 0.0002$ \\
$\mathbf{8 i}$ & $2.56 \pm 0.01$ & $0.0288 \pm 0.0001$ & Vit C & $0.07 \pm 0.00$ & $1 \pm 0$ \\
$\mathbf{8 j}$ & $1.99 \pm 0.01$ & $0.0369 \pm 0.0003$ & & & \\
\hline
\end{tabular}

a The data are expressed as the mean \pm SD, $n=4$.

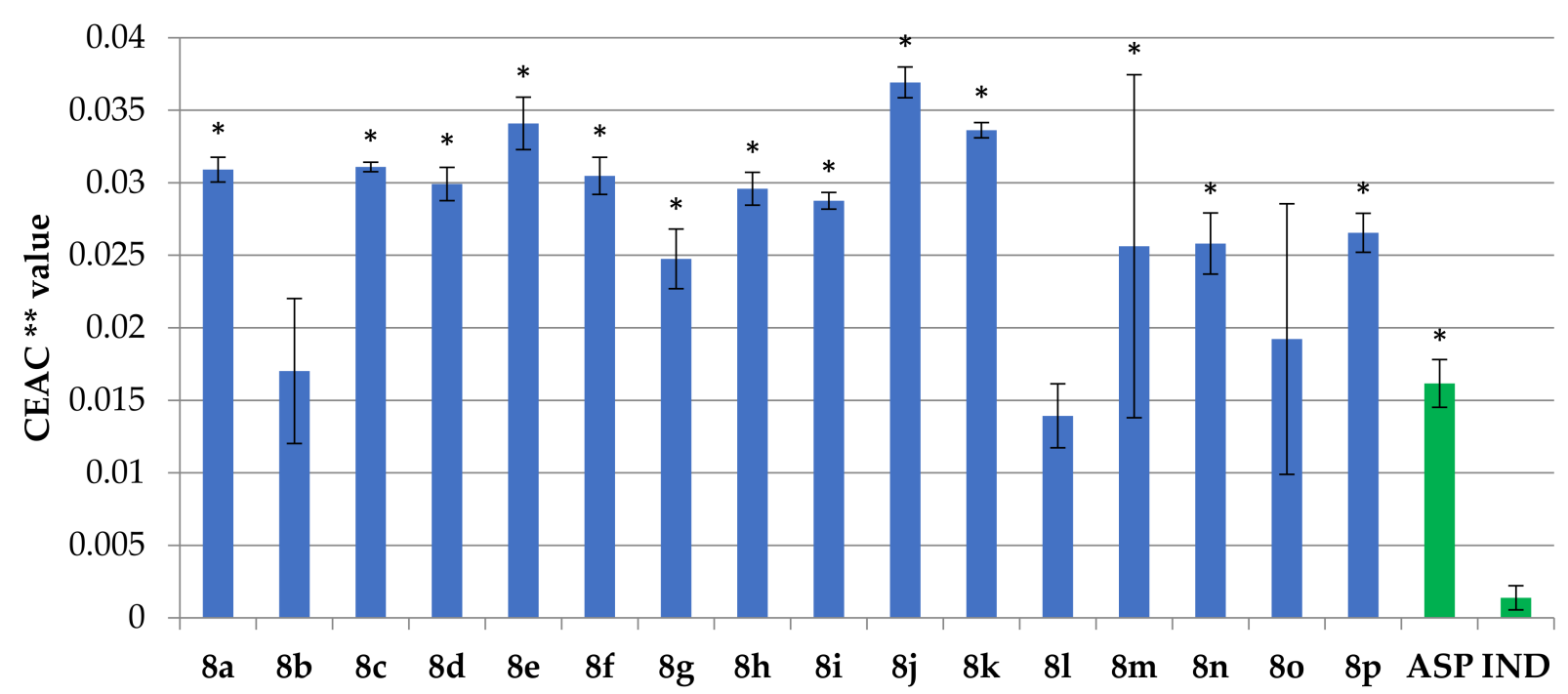

Figure 4. The DPPH radical scavenging activity of NO-IND-OXDs (8a-p) and of reference drugs (IND, ASP) expressed as CEAC values ( ${ }^{*}$ significant differences than $\mathrm{ASP}(p<0.05) ;{ }^{* *}$ mean $\left.\pm \mathrm{SD}, n=4\right)$.

Using one-way ANOVA test, a statistically significant difference $(F(17,71)=164.778$, $p<0.05)$ between tested compounds (8a-p, ASP, IND) was highlighted. Post hoc comparisons using the Tukey HSD test revealed that all tested compounds have significantly improved antioxidant effect in reference to IND $(p<0.05)$. Moreover, it was noted that most of the tested compounds (except $\mathbf{8 b}, \mathbf{8 1}$ and $\mathbf{8 0}$ ) are significantly more active than aspirin (ASP) $(p<0.05)$. It can be appreciated that the majority of compounds are from 10.0 to 26.7 times more active than IND $(\mathrm{CEAC}=0.0014 \pm 0.0002)$ and from 1.5 to 2.3 times more active than ASP $($ CEAC $=0.0162 \pm 0.0004)$. Compared to vitamin $C$, used as a positive control, all tested compounds were less active, the CEAC values being less than 1 .

Moreover, it was reported that 1,3,4-oxadiazole scaffold has been found to be a potent antioxidant pharmacophore, and chemical modulation of the conventional drugs with this scaffold resulted in a synergistic effect, enhancing the antioxidant efficiency of the parent drug $[47,49,50,83,84]$. In our research, the presence of 1,3,4-oxadiazole scaffold significantly enhanced antioxidant activity for the majority of the NO-IND-OXDs.

\subsection{Thermal Denaturation of Serum Proteins}

The Mizushima's test, which is based on thermal denaturation of proteins, was used to predict the anti-inflammatory effects of the new NO-IND-OXDs 8a-p [85]. The NSAIDs are strong ligands to enzymatic and non-enzymatic proteins and it has been proven that their hydrophilic part, as an anionic radical, interacts with the polar amino acids of proteins while their lipophilic part is fixed into the hydrophobic site of proteins [86]. As a result of these 
interactions, various changes in the native structure of the proteins, such as modification of the secondary, tertiary or quaternary structure without breaking the covalent bonds, occur $[87,88]$. It was reported that the native state of the proteins is stable in hydrophobic solvents, whereas the polar organic solvents such as trifluoroethanol, DMFA or DMSO affect their structure, the degree of injury being concentration-dependent $[89,90]$.

When a compound is incubated with non-enzymatic proteins, such as bovine serum albumin (BSA), an increase in optical density will occur because of protein precipitation [88]. Because the tested compounds 8a-p are less soluble in water, DMSO $(40 \%)$ was used as solvent. The results showed that BSA $(0.2 \%$ in $0.9 \% \mathrm{NaCl} / \mathrm{DMSO}=6 / 4)$ remained stable after incubation at $38{ }^{\circ} \mathrm{C}$ for $5 \mathrm{~h}$ and no turbidity was observed. In similar conditions, the compounds $8 \mathbf{a}-\mathbf{p}$ cause an intense precipitation of BSA and a cloudy solution was obtained. The results, expressed as denaturation effect (\%) at different concentration $(25 \mu \mathrm{M}, 50 \mu \mathrm{M}$ and $100 \mu \mathrm{M})$ in reference to positive control $(100 \%$ denaturation of BSA induced by $0.1 \mathrm{M}$ hydrochloric acid) are presented in Figure 5.

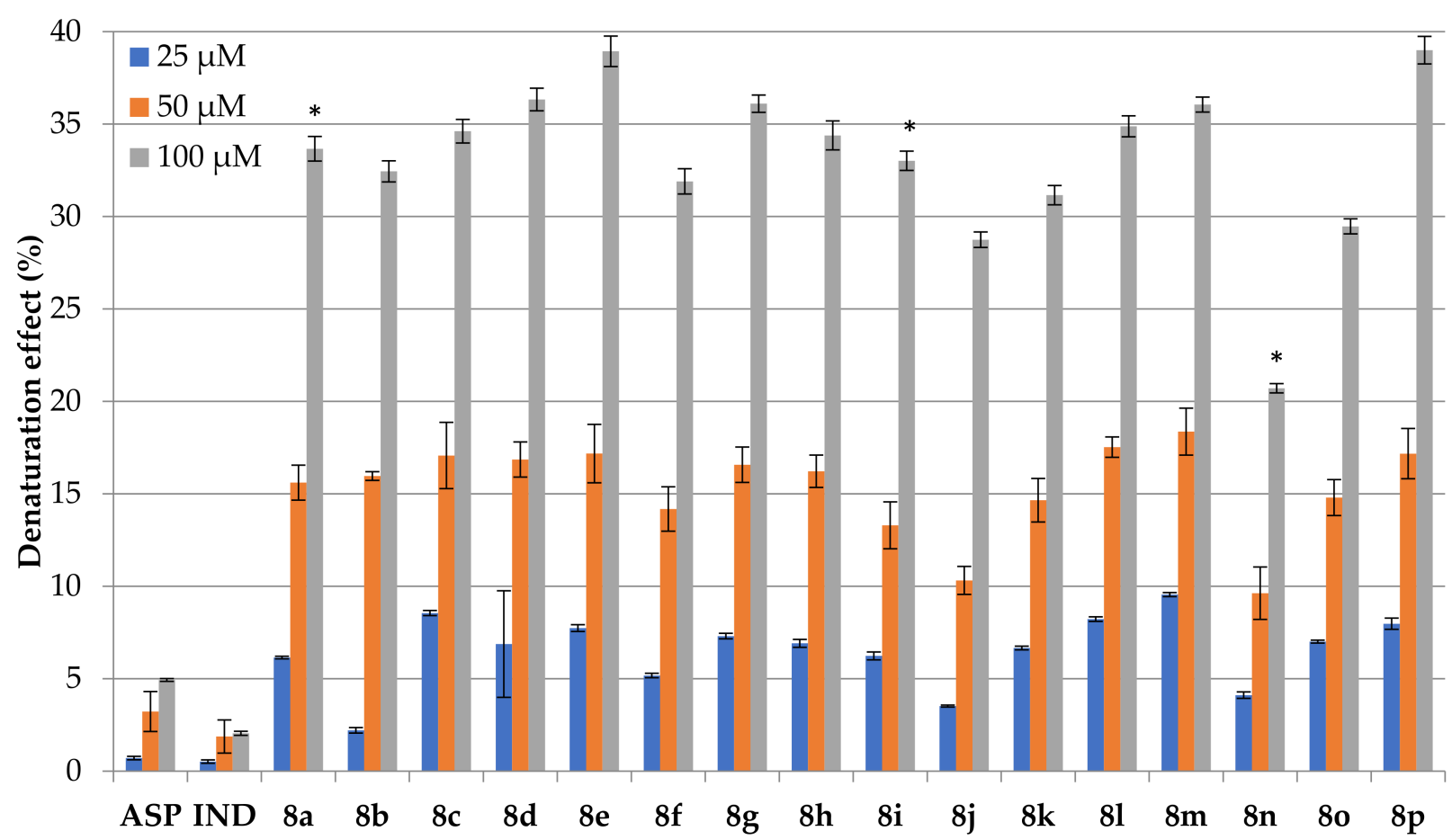

Figure 5. The effects of NO-IND-OXDs $(\mathbf{8} \mathbf{a}-\mathbf{p})$ and of reference drugs (IND, ASP) on BSA denaturation process $\left({ }^{*} \mathbf{8} \mathbf{n}<\mathbf{8 a}\right.$ and $8 \mathbf{i}, p<0.05)$.

Using two-way ANOVA test, it was determined that there is a statistically significant interaction $(F(30,144)=2060.794, p<0.05)$ between concentration and structure of compounds referring to the denaturation effect. It was noted that the tested compounds $\mathbf{8 a}-\mathbf{p}$ were able to increase the albumin denaturation in a concentration-dependent manner; the higher concentration increases the denaturation effect. For example, the compounds $8 \mathbf{a}, \mathbf{8} \mathbf{i}$ and $8 \mathbf{n}$ showed a statistically significant increase of denaturation effect $(p<0.05)$ with concentration increasing, but at the same level of each concentration there was no difference ( $p=0.150$ ) between $\mathbf{8 a}$ and $\mathbf{8} \mathbf{i}$ compared with $\mathbf{8 n}$, for which the denaturation effect increased significantly less $(p<0.05)$. At $100 \mu \mathrm{M}$, compound $\mathbf{8 n}$ was less active $(20.7 \pm 0.4 \%)$ compared with $\mathbf{8 a}(33.7 \pm 1.0 \%)$ and $\mathbf{8 i}(33.0 \pm 0.9 \%)$. Therefore, in relation to chemical structure, we can also note that the presence of nitrate ester moiety in para and meta positions was more favorable than ortho, 8a (4-oxy-ethylnitrate) and 8i (3-oxy-ethylnitrate) being more active than $8 \mathrm{n}$ (2-oxy-ethylnitrate). 
Compared to the reference drugs, IND and ASP, which have a denaturation effect of less than $5 \%$, all the tested compounds were shown to be more active, with the value of the denaturation effect ranging between $20 \%$ and $40 \%$ (at $100 \mu \mathrm{M}$ ).

The results of our study also revealed that the presence of halogens $(\mathrm{F}, \mathrm{Cl}, \mathrm{Br})$ or $\mathrm{NO}_{2}$ on phenoxy-ethylnitrate moiety, as well as the elongation of distance between oxadiazole moiety and aromatic ring from methylene group (8a) to ethylene group $(\mathbf{8 p})$ increases the denaturation effect and thus promotes interaction with non-enzymatic proteins.

In addition, it is known that ASP is able to produce acetylation of proteins (such as human serum albumin, fibrinogen, p53, cellular protein) and the ASP therapy is associated with the rise of the anti-acetylated serum albumin antibodies [91-93]. It was also reported that these interactions could be used to predict the anti-inflammatory properties of the compounds [94]. The results of our study revealed that the chemical modulation of free carboxylic group of IND with oxadiazole moiety enhances the drug-protein interactions that are in agreement with previous in silico results, which showed good interaction with COX enzyme sites.

\subsection{In Vitro Nitric Oxide Release Measurement}

For detection and quantification of NO released by NO-IND-OXDs $\mathbf{8 a - p}$, a modified colorimetric Griess assay was used, based on decomposition of nitrate ester moiety in presence of $\mathrm{Hg}^{2+}$ and thiol-based compounds. The study was performed using different NO donors as reference, such as sodium nitropruside (SNP), nitroglycerine (NTG) and $S$-nitroso- $N$-acetyl-penicillamine (SNAP), which belong to different classes. SNP is a metal nitrosyl compound that spontaneously releases NO at physiological pH, NTG is a representative organic nitrate which requires specific thiol and/or enzymatic activation to generate NO and SNAP is an S-nitrosothiol which is rapidly decomposed in the presence of metal ions, such as $\mathrm{Cu}^{+}, \mathrm{Fe}^{2+}, \mathrm{Hg}^{2+}$ and $\mathrm{Ag}^{+}$.

In order to appropriately simulate the body conditions, the NO releasing from the NO-IND-OXDs 8a-p was evaluated in intestinal (PBS, PBS-GSH) and gastric $(\mathrm{HCl}$, $\mathrm{HCl}-\mathrm{GSH}$ ) environmental conditions, respectively, and the results are presented in Figures 6 and 7. As expected, using one-way ANOVA analysis, a statistically significant difference $(F(2,93)=60.729, p<0.05)$ between NO donors, used as reference, was found. In addition, Tukey HSD post hoc comparison test indicated that the NO percentage released for SNAP $(52.70 \pm 16.82)$ was significantly higher than the SNP $(3.97 \pm 1.21)$ and NTG $(0.49 \pm 0.18)$ (Figure 7). There was no statistically significant interaction between experimental conditions (PBS, PBS-GSH, HCl, HCl-GSH) and used Griess reagents (NED-SULF/SULF-HgCl ${ }_{2}$ ), as determined by two-way Anova $(F(3.88)=1.029$, $p=0.384$ ). Moreover, the presence of $\mathrm{Hg}^{2+}$ in Griess reagents increased by a statistically significant value the mean of NO released $(p=0.002)$, but changing the experimental conditions did not significantly influence $(p=0.669)$ the quantified amount of NO. These results suggest that the GSH, present in the experimental medium, fixes the free NO, forming a stable $S$-nitrosothiol (GS-NO) and so prevents the oxidation of NO into stable $\mathrm{NO}_{3}{ }^{-}$, which cannot be detected using Griess reagents. It was also noted that the $\mathrm{Hg}^{2+}$ produces the cleavage of the $\mathrm{S}-\mathrm{NO}$ bond from SNAP. Therefore, the GSH and $\mathrm{Hg}^{2+}$ are appropriate reagents to measure the total amount of released NO. In addition, the experimental $\mathrm{pH}$ has no significant influence on $\mathrm{NO}$ release from $\mathrm{NO}$ donors.

Referring to the tested compounds $\mathbf{8 a}-\mathbf{p}$, no statistically significant interaction $(F(3,504)=0.465, p=0.707)$ between Griess reagents and experimental conditions, applied to study the NO release, was noted. Moreover, it was noted that the adding of $\mathrm{HgCl}_{2}$ to SULF solution did not significantly increase the total amount of NO released from nitrate ester moiety $(F(1,504)=0.122, p=0.727)$. A statistically significant interaction between structure of compounds $8 \mathbf{a}-\mathbf{p}$ and experimental conditions $(F(45,448)=69.945, p<0.05)$ was noted. All these results suggest that the presence of $\mathrm{Hg}^{2+}$ in Griess reagents did not influence the amount of NO released by NO-IND-OXDs 
but it was strongly influenced by experimental conditions ( $\mathrm{pH}$ and GSH presence) and the structure of tested compounds.

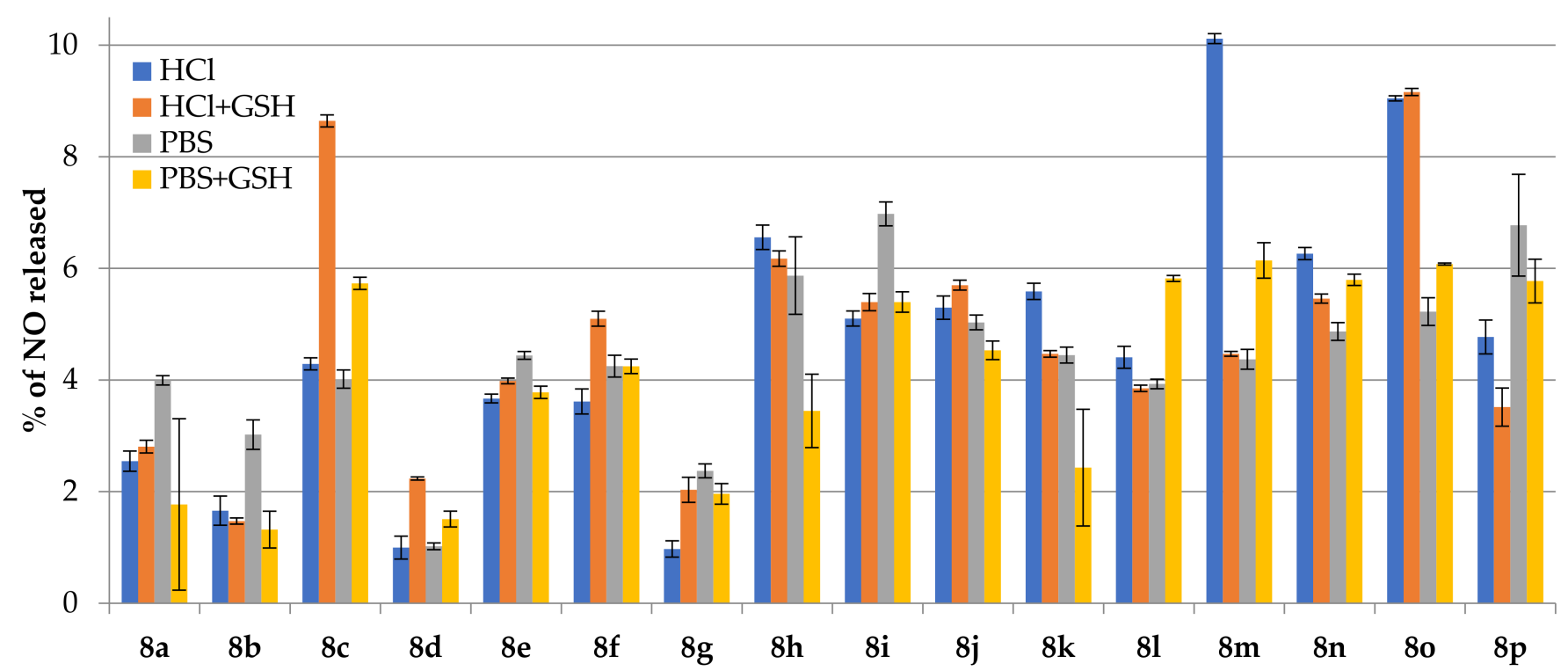

Figure 6. The percentage (\%) of NO released by NO-IND-OXDs 8a-p in different experimental conditions (PBS, PBS-GSH, $\mathrm{HCl}, \mathrm{HCl}-\mathrm{GSH})$, relative to theoretical maximum release of $1.0 \mathrm{~mol} \mathrm{NO} / \mathrm{mol}$ tested compound) $(n=4)$.

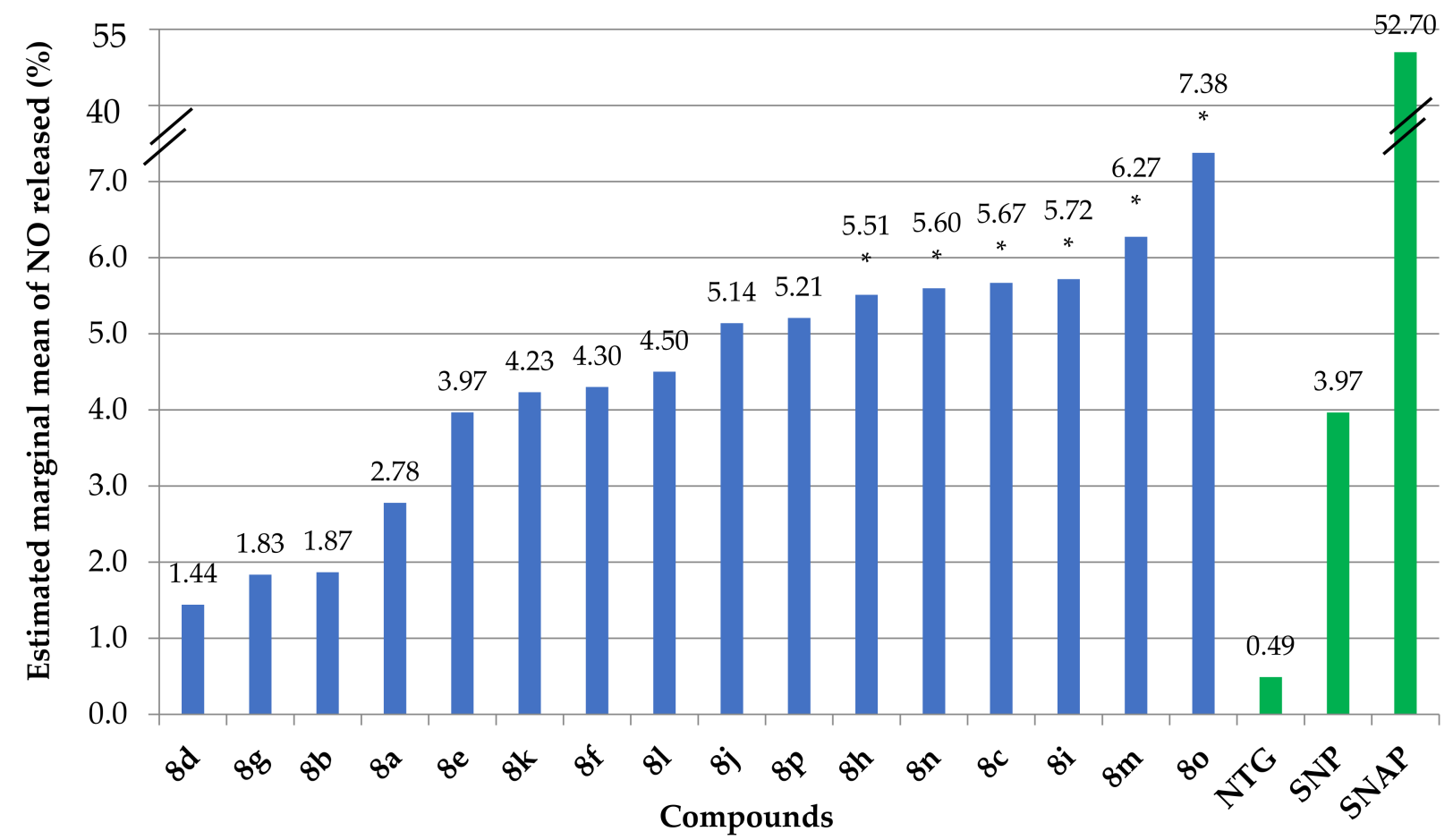

Figure 7. The estimated marginal means of NO (\%) release by NO-IND-OXDs (8a-p) compared to reference NO donors (NTG, SNP, SNAP) $\left({ }^{*}\right.$ significantly more NO released than SNP, $\left.p<0.05\right)$.

By estimated marginal mean of $\mathrm{NO}$ released it was shown that all of the tested compounds 8a-p released more NO compared with NTG (Figure 7). Using one-way ANOVA test it was determined that there is a statistically significant difference $(F(16,255)=33.054$, $p<0.05)$ between tested compounds. Post hoc comparisons using the Tukey HSD test revealed that the tested compounds $8 \mathrm{~h}, \mathbf{8 n}, \mathbf{8 c}, \mathbf{8 i}, \mathbf{8 m}$ and $8 \mathrm{o}$, respectively, released significantly more NO than SNP $(p<0.05)$. It was noted that the amount of NO released is 
influenced by the position of nitrate ester moiety on aromatic ring and by the number and type of the substituents on aromatic ring. The most proper positions of nitrate ester moiety are meta and ortho, 8i (3-oxy-ethylnitrate) and 8n (2-oxy-ethylnitrate) being more active than 8a (4-oxy-ethylnitrate). In addition, the elongation of distance between oxadiazole moiety and aromatic ring from methylene group (8a) to ethylene group (8p) increase the percentage of $\mathrm{NO}$ released.

It was reported that $\mathrm{NO}$, at low level (less than $300 \mathrm{nM}$ ) exerts beneficial effects (e.g., anti-inflammatory, antioxidant, wound healing effects) while at high level (1000-3000 nM) it has pro-inflammatory effects in pathological conditions [40,95]. The NO-IND-OXDs could act as new drugs because of their balanced inhibition of COX enzymes and their ability to release $\mathrm{NO}$ at a submicromolar level, which was proven to be beneficial for inflammation conditions.

\section{Materials and Methods}

\subsection{Chemistry}

\subsubsection{General Information}

All chemicals used in this research were of analytical grade or HPLC p.a. quality, certified by the commercially available sources and were used without further purification unless otherwise specified. The anhydrous solvents were distilled or dried in accordance with standard procedures before use: dichloromethane was distilled under $\mathrm{P}_{2} \mathrm{O}_{5}$ and acetonitrile was dried on cartridge by a GT S100 station. The reaction progress was monitored by thin layer chromatography (TLC), using $2 \times 5 \mathrm{~cm}$ precoated UV fluorescent silicagel aluminum plate (type Merck 60 F254) and UV lamp exposure (254 nm). The synthesized compounds were purified by flash column chromatography on silicagel 60 $\left(0.063-0.200 \mathrm{~mm}\right.$, Merck), using appropriate solvents. The ${ }^{1} \mathrm{H}$ NMR $(250 \mathrm{MHz}$ or $400 \mathrm{MHz})$, ${ }^{13} \mathrm{C}$ NMR $(63 \mathrm{MHz}$ or $101 \mathrm{MHz})$ and ${ }^{19} \mathrm{~F}$ NMR $(376 \mathrm{MHz})$ spectra were recorded on Bruker Avance DPX250 (250, $131 \mathrm{MHz})$ or Bruker Avance II (400 MHz) spectrometer. Chemical shifts $(\delta \mathrm{H}, \delta \mathrm{C})$ and coupling constant values $(J)$ were given in $\mathrm{ppm}$ and $\mathrm{Hz}$, respectively. The chemical shifts were referenced to tetramethylsilane (TMS, $\delta \mathrm{H}=0$, $\delta \mathrm{C}=0)$ in $\mathrm{CDCl}_{3}$ and DMSO- $d_{6}$. The standard abbreviation for signals is as follows: singlet (s), doublet (d), doublet of doublets (dd), doublet of doublets of doublets (ddd), triplet $(\mathrm{t})$, triplet of doublets $(\mathrm{dt})$, quartet $(\mathrm{q})$, hexuplet $(\mathrm{h})$, multiplet $(\mathrm{m})$. For detailed peak assignments, 2D NMR analysis: gradient-selected correlation spectroscopy (gCOSY), gradient-selected heteronuclear multiple bond coherence (gHMBC) and gradient-selected heteronuclear multiple quantum coherence $(\mathrm{gHMQC})$ were used. High-resolution mass spectrometry (HRMS) was recorded on a Bruker maXis mass spectrometer within the "Research Federation" platform between ICOA and CBM (FR2708). The samples were ionized by electrospray ion source (ESI), using $\mathrm{N}_{2}$ for nebulization (pressure of 0.6 bar) and drying (flow of $7 \mathrm{~L} / \mathrm{min}$, temperature of $200{ }^{\circ} \mathrm{C}$ ). The capillary potential was set at $4500 \mathrm{~V}$ and collision cell RF at $1800.0 \mathrm{Vpp}$. The ion polarities were positive and were analyzed using scanning range between $50-2500 \mathrm{~m} / \mathrm{z}$. Melting points (m.p.) were measured on a Kofler heating bench. A microplate reader (Tecan Sunrise Remote Microplate Reader TW /ML-Abbott F039306) and Hanna Model Pack pH 21 digital pHmeter were also used.

3.1.2. General Procedure for the Synthesis of the (bromoethoxy)benzaldehyde Derivatives (2a-o)

The intermediates $\mathbf{2 a - 0}$ were obtained by reaction of hydroxybenzaldehydes 1a-o (1.0 Eq.) in acetonitrile ( $150 \mathrm{~mL}$ for $15 \mathrm{mmol}$ scale) with 1,2-dibromoethane (10.0 Eq.) in the presence of potassium carbonate (2.0 Eq.), according to the method reported in the literature [96-98], which was reported in our previous paper [99].

3.1.3. General Procedure for the Synthesis of the (bromoethoxy)aromatic Alcohol Derivatives ( $\mathbf{3 a}-\mathbf{p})$

To a solution of appropriate (bromoethoxy)benzaldehydes 2a-o (1.0 Eq.), in dry THF ( $30 \mathrm{~mL}$ for $10 \mathrm{mmol}$ scale), sodium borohydride $\left(1.1 \mathrm{Eq}\right.$.) was added at $0^{\circ} \mathrm{C}$ and the mixture 
was stirred at room temperature for $12-24 \mathrm{~h}$, according to the method reported in the literature [100-103]. Then, the excess of sodium borohydride was quenched by addition of $1 \mathrm{M} \mathrm{HCl}$ and extracted twice with EtOAc $(30 \mathrm{~mL})$. The combined organic layers were washed with water and brine, dried over anhydrous magnesium sulfate, filtered and concentrated under reduced pressure. To obtain 2-(4-(2-bromoethoxy)phenyl)ethanol (3p), 4-(2-hydroxyethyl)phenol (2p) (1.0 Eq.) was reacted with 1,2-dibromoethane (10.0 Eq.) in $\mathrm{CH}_{3} \mathrm{CN}$ (50 mL for $15 \mathrm{mmol}$ scale) in the presence of potassium carbonate (2.0 Eq.). The resulting mixture was heated to reflux for $20 \mathrm{~h}$. After cooling to room temperature, distilled water $(100 \mathrm{~mL})$ was added and the crude product was extracted with diethyl ether $(3 \times 50 \mathrm{~mL})$.

The combined organic layers were washed with distilled water $(50 \mathrm{~mL})$, brine $(50 \mathrm{~mL})$ and dried over anhydrous magnesium sulfate. The crude products were purified by flash column chromatography on silicagel with petroleum ether/EtOAc or $\mathrm{CH}_{2} \mathrm{Cl}_{2} / \mathrm{CH}_{3} \mathrm{OH}$ to give the pure compounds $3 \mathbf{a}-\mathbf{p}$.

(4-(2-Bromoethoxy)phenyl)methanol (3a): white solid, yield $95 \%$, m.p. $73-75^{\circ} \mathrm{C} ;{ }^{1} \mathrm{H}$ NMR $\left(250 \mathrm{MHz}, \mathrm{DMSO}-\mathrm{d}_{6}\right) \delta=3.68-3.80(\mathrm{~m}, 2 \mathrm{H}), 4.18-4.30(\mathrm{~m}, 2 \mathrm{H}), 4.37(\mathrm{~d}, J=5.7 \mathrm{~Hz}, 2 \mathrm{H}), 5.01$ $(\mathrm{t}, J=5.7 \mathrm{~Hz}, 1 \mathrm{H}), 6.81-6.93(\mathrm{~m}, 2 \mathrm{H}), 7.13-7.25(\mathrm{~m}, 2 \mathrm{H}) ;{ }^{13} \mathrm{C}$ NMR $\left(101 \mathrm{MHz}, \mathrm{DMSO}-d_{6}\right)$ $\delta=31.5\left(\mathrm{CH}_{2}\right), 62.5\left(\mathrm{CH}_{2} \mathrm{OH}\right), 67.7\left(\mathrm{CH}_{2}\right), 114.3\left(2 \mathrm{CH}_{\mathrm{Ar}}\right), 127.9\left(2 \mathrm{CH}_{\mathrm{Ar}}\right), 135.1\left(\mathrm{C}_{\mathrm{q}}\right), 156.8$ $\left(\mathrm{C}_{\mathrm{q}}\right)$; HRMS (ESI-MS) m/z calcd for $\mathrm{C}_{9} \mathrm{H}_{11} \mathrm{BrNaO}_{2}[\mathrm{M}+\mathrm{Na}]^{+}:$252.9840, found: 252.9835; Rf $\left(\mathrm{CH}_{2} \mathrm{Cl}_{2} / \mathrm{CH}_{3} \mathrm{OH}=9.8 / 0.2\right) 0.53$, according to [104].

(4-(2-Bromoethoxy)-3-fluorophenyl)methanol (3b): pale yellow oil, yield 93\%; ${ }^{1} \mathrm{H} \mathrm{NMR}$ $\left(250 \mathrm{MHz}, \mathrm{DMSO}-d_{6}\right) \delta=3.76-3.84(\mathrm{~m}, 2 \mathrm{H}), 4.32-4.40(\mathrm{~m}, 2 \mathrm{H}), 4.42(\mathrm{dd}, J=5.7 \mathrm{~Hz}, 0.7 \mathrm{~Hz}$, $2 \mathrm{H}), 5.19(\mathrm{t}, J=5.7 \mathrm{~Hz}, 1 \mathrm{H}), 7.00-7.22(\mathrm{~m}, 3 \mathrm{H}) ;{ }^{13} \mathrm{C} \mathrm{NMR}\left(101 \mathrm{MHz}, \mathrm{DMSO}-d_{6}\right) \delta=31.2$ $\left(\mathrm{CH}_{2}\right), 61.9\left(\mathrm{CH}_{2} \mathrm{OH}\right), 69.0\left(\mathrm{CH}_{2}\right), 114.2\left(\mathrm{~d}, J=18.2 \mathrm{~Hz}, \mathrm{CH}_{\mathrm{Ar}}\right), 115.3\left(\mathrm{~d}, J=1.9 \mathrm{~Hz}, \mathrm{CH}_{\mathrm{Ar}}\right)$, $122.4\left(\mathrm{~d}, J=3.4 \mathrm{~Hz}, \mathrm{CH}_{\mathrm{Ar}}\right), 136.6\left(\mathrm{~d}, J=5.6 \mathrm{~Hz}, \mathrm{C}_{\mathrm{q}}\right), 144.3\left(\mathrm{~d}, J=10.9 \mathrm{~Hz}, \mathrm{C}_{\mathrm{q}}\right), 151.6(\mathrm{~d}$, $\left.J=243.9 \mathrm{~Hz}, \mathrm{C}_{\mathrm{q}} \mathrm{F}\right) ;{ }^{19} \mathrm{~F}$ NMR $\left(376 \mathrm{MHz}, \mathrm{DMSO}-d_{6}\right) \delta=-134.7(\mathrm{dd}, J=12.5 \mathrm{~Hz}, 8.6 \mathrm{~Hz})$; HRMS (ESI-MS) $\mathrm{m} / \mathrm{z}$ calcd for $\mathrm{C}_{9} \mathrm{H}_{10} \mathrm{BrFNaO}_{2}[\mathrm{M}+\mathrm{Na}]^{+}$: 270.9746, found: 270.9740; $\mathrm{Rf}$ $\left(\mathrm{CH}_{2} \mathrm{Cl}_{2} / \mathrm{CH}_{3} \mathrm{OH}=9.8 / 0.2\right) 0.61$.

(4-(2-Bromoethoxy)-3-chlorophenyl)methanol (3c): pale yellow oil, yield 97\%; ${ }^{1} \mathrm{H}$ NMR $\left(400 \mathrm{MHz}, \mathrm{DMSO}-d_{6}\right) \delta=3.75-3.83(\mathrm{~m}, 2 \mathrm{H}), 4.35-4.40(\mathrm{~m}, 2 \mathrm{H}), 4.42(\mathrm{~d}, J=5.8 \mathrm{~Hz}, 2 \mathrm{H})$, $5.19(\mathrm{t}, J=5.8 \mathrm{~Hz}, 1 \mathrm{H}), 7.11(\mathrm{~d}, J=8.4 \mathrm{~Hz}, 1 \mathrm{H}), 7.22(\mathrm{dd}, J=8.4 \mathrm{~Hz}, 2.0 \mathrm{~Hz}, 1 \mathrm{H}), 7.37(\mathrm{~d}$, $J=2.1 \mathrm{~Hz}, 1 \mathrm{H}) ;{ }^{13} \mathrm{C}$ NMR $\left(101 \mathrm{MHz}, \mathrm{DMSO}-d_{6}\right) \delta=31.0\left(\mathrm{CH}_{2}\right), 61.8\left(\mathrm{CH}_{2} \mathrm{OH}\right), 68.8\left(\mathrm{CH}_{2}\right)$, 114.1 $\left(\mathrm{CH}_{\mathrm{Ar}}\right), 121.2\left(\mathrm{C}_{\mathrm{q}}\right), 126.3\left(\mathrm{CH}_{\mathrm{Ar}}\right), 128.1\left(\mathrm{CH}_{\mathrm{Ar}}\right), 136.6\left(\mathrm{C}_{\mathrm{q}}\right), 152.0\left(\mathrm{C}_{\mathrm{q}}\right)$. HRMS (ESIMS) $\mathrm{m} / \mathrm{z}$ calcd for $\mathrm{C}_{9} \mathrm{H}_{10} \mathrm{BrClNaO}_{2}[\mathrm{M}+\mathrm{Na}]^{+}:$286.9450, found: 286.9835; Rf (petroleum ether $/ \mathrm{EtOAC}=5 / 5) 0.58$.

(4-(2-Bromoethoxy)-3,5-dichlorophenyl)methanol (3d): white solid, yield 96\%, m.p. 65-67 ${ }^{\circ} \mathrm{C} ;{ }^{1} \mathrm{H}$ NMR $\left(250 \mathrm{MHz}, \mathrm{DMSO}-d_{6}\right) \delta=3.72-3.86(\mathrm{~m}, 2 \mathrm{H}), 4.18-4.34(\mathrm{~m}, 2 \mathrm{H}), 4.46$ $(\mathrm{d}, J=5.8 \mathrm{~Hz}, 2 \mathrm{H}), 5.40(\mathrm{t}, J=5.8 \mathrm{~Hz}, 1 \mathrm{H}), 7.40(\mathrm{~s}, 2 \mathrm{H}) ;{ }^{13} \mathrm{C}$ NMR $(101 \mathrm{MHz}, \mathrm{DMSO}-$ $\left.d_{6}\right) \delta=31.2\left(\mathrm{CH}_{2}\right), 61.3\left(\mathrm{CH}_{2} \mathrm{OH}\right), 72.9\left(\mathrm{CH}_{2}\right), 126.8\left(2 \mathrm{CH}_{\mathrm{Ar}}\right), 128.0\left(\mathrm{C}_{\mathrm{q}}\right), 141.3\left(\mathrm{C}_{\mathrm{q}}\right)$, $148.5\left(\mathrm{C}_{\mathrm{q}}\right)$; HRMS (ESI-MS) $\mathrm{m} / \mathrm{z}$ calcd for $\mathrm{C}_{9} \mathrm{H}_{9} \mathrm{BrCl}_{2} \mathrm{NaO}_{2}[\mathrm{M}+\mathrm{Na}]^{+}:$320.9061, found: 320.9055; $\mathrm{Rf}\left(\mathrm{CH}_{2} \mathrm{Cl}_{2} / \mathrm{CH}_{3} \mathrm{OH}=9.8 / 0.2\right) 0.64$.

(4-(2-Bromoethoxy)-3-nitrophenyl)methanol (3e): brown oil, yield 86\%; ${ }^{1} \mathrm{H} \mathrm{NMR}(400 \mathrm{MHz}$, DMSO- $\left.d_{6}\right) \delta=3.72-3.84(\mathrm{~m}, 2 \mathrm{H}), 4.41-4.56(\mathrm{~m}, 4 \mathrm{H}), 5.35(\mathrm{t}, J=5.7 \mathrm{~Hz}, 1 \mathrm{H}), 7.34(\mathrm{~d}, J=8.7 \mathrm{~Hz}$, $1 \mathrm{H}), 7.57(\mathrm{dd}, J=8.6 \mathrm{~Hz}, 2.1 \mathrm{~Hz}, 1 \mathrm{H}), 7.79(\mathrm{~d}, J=2.1 \mathrm{~Hz}, 1 \mathrm{H}) ;{ }^{13} \mathrm{C}$ NMR $\left(101 \mathrm{MHz}, \mathrm{DMSO}-d_{6}\right)$ $\delta=30.7\left(\mathrm{CH}_{2}\right), 61.4\left(\mathrm{CH}_{2} \mathrm{OH}\right), 69.4\left(\mathrm{CH}_{2}\right), 115.4\left(\mathrm{CH}_{\mathrm{Ar}}\right), 122.6\left(\mathrm{CH}_{\mathrm{Ar}}\right), 132.2\left(\mathrm{CH}_{\mathrm{Ar}}\right), 135.9$ $\left(\mathrm{C}_{\mathrm{q}}\right), 139.4\left(\mathrm{C}_{\mathrm{q}}\right), 149.3\left(\mathrm{C}_{\mathrm{q}}\right)$; HRMS (ESI-MS) $\mathrm{m} / \mathrm{z}$ calcd for $\mathrm{C}_{9} \mathrm{H}_{11} \mathrm{BrNO}_{4}[\mathrm{M}+\mathrm{H}]^{+}:$275.9871, found: $275.9865 ; \mathrm{Rf}$ (petroleum ether $/ \mathrm{EtOAc}=4 / 6) 0.56$.

(4-(2-Bromoethoxy)-3-methoxyphenyl)methanol (3f): white solid, yield $91 \% ;{ }^{1} \mathrm{H} \mathrm{NMR}$ $\left(250 \mathrm{MHz}, \mathrm{DMSO}-\mathrm{d}_{6}\right) \delta=3.70-3.82(\mathrm{~m}, 2 \mathrm{H}), 3.77(\mathrm{~s}, 3 \mathrm{H}), 4.26(\mathrm{~m}, 2 \mathrm{H}), 4.42(\mathrm{~d}, J=5.7 \mathrm{~Hz}$, $2 \mathrm{H}), 5.08(\mathrm{t}, J=5.7 \mathrm{~Hz}, 1 \mathrm{H}), 6.81(\mathrm{ddt}, J=8.1 \mathrm{~Hz}, 1.9 \mathrm{~Hz}, 0.72,1 \mathrm{H}), 6.92(\mathrm{~d}, J=8.1 \mathrm{~Hz}, 1 \mathrm{H})$, $6.95(\mathrm{~d}, J=1.9 \mathrm{~Hz}, 1 \mathrm{H}) ;{ }^{13} \mathrm{C}$ NMR $\left(101 \mathrm{MHz}, \mathrm{DMSO}-d_{6}\right) \delta=31.5\left(\mathrm{CH}_{2}\right), 55.5\left(\mathrm{CH}_{3}\right), 62.7$ $\left(\mathrm{CH}_{2} \mathrm{OH}\right), 69.0\left(\mathrm{CH}_{2}\right), 111.1\left(\mathrm{CH}_{\mathrm{Ar}}\right), 114.2\left(\mathrm{CH}_{\mathrm{Ar}}\right), 118.6\left(\mathrm{CH}_{\mathrm{Ar}}\right), 136.3\left(\mathrm{C}_{\mathrm{q}}\right), 146.0\left(\mathrm{C}_{\mathrm{q}}\right), 149.1$ $\left(\mathrm{C}_{\mathrm{q}}\right)$;. HRMS (ESI-MS) $\mathrm{m} / \mathrm{z}$ calcd for $\mathrm{C}_{10} \mathrm{H}_{13} \mathrm{BrNaO}_{3}[\mathrm{M}+\mathrm{Na}]^{+}$: 282.9946, found: 282.9940; $\mathrm{Rf}$ (petroleum ether $/ \mathrm{EtOAc}=3 / 7$ ) 0.53, according to $[105,106]$. 
(4-(2-Bromoethoxy)-3-ethoxyphenyl)methanol (3g): white solid, yield 93\%, m.p. $53-55{ }^{\circ} \mathrm{C}$; ${ }^{1} \mathrm{H}$ NMR $\left(250 \mathrm{MHz}, \mathrm{DMSO}-d_{6}\right) \delta=1.32(\mathrm{t}, J=6.9 \mathrm{~Hz}, 3 \mathrm{H}), 3.75(\mathrm{dd}, J=6.0 \mathrm{~Hz}, 5.2 \mathrm{~Hz}, 2 \mathrm{H})$, $4.03(\mathrm{q}, J=6.9 \mathrm{~Hz}, 2 \mathrm{H}), 4.27(\mathrm{t}, J=6.0 \mathrm{~Hz}, 5.22 \mathrm{~Hz}, 2 \mathrm{H}), 4.41(\mathrm{~d}, J=5.7 \mathrm{~Hz}, 0.6 \mathrm{~Hz}, 2 \mathrm{H})$, $5.07(\mathrm{t}, J=5.7 \mathrm{~Hz}, 1 \mathrm{H}), 6.81(\mathrm{ddt}, J=8.0 \mathrm{~Hz}, 1.8 \mathrm{~Hz}, 0.6 \mathrm{~Hz}, 1 \mathrm{H}), 6.89-6.96(\mathrm{~m}, 2 \mathrm{H}) ;{ }^{13} \mathrm{C}$ NMR (101 MHz, DMSO-d $\left.d_{6}\right) \delta=14.8\left(\mathrm{CH}_{3}\right), 31.5\left(\mathrm{CH}_{2}\right), 62.6\left(\mathrm{CH}_{2} \mathrm{OH}\right), 64.0\left(\mathrm{CH}_{2}\right), 69.2$ $\left(\mathrm{CH}_{2}\right), 112.8\left(\mathrm{CH}_{\mathrm{Ar}}\right), 115.1\left(\mathrm{CH}_{\mathrm{Ar}}\right), 118.8\left(\mathrm{CH}_{\mathrm{Ar}}\right), 136.5\left(\mathrm{C}_{\mathrm{q}}\right), 146.4\left(\mathrm{C}_{\mathrm{q}}\right), 148.5\left(\mathrm{C}_{\mathrm{q}}\right)$; HRMS (ESI-MS) $\mathrm{m} / \mathrm{z}$ calcd for $\mathrm{C}_{11} \mathrm{H}_{15} \mathrm{BrNaO}_{3}[\mathrm{M}+\mathrm{Na}]^{+}$: 297.0102, found: 297.0096; Rf (petroleum ether $/ \mathrm{EtOAc}=3 / 7)$ 0.55, according to [107].

(4-(2-Bromoethoxy)-3,5-dimethoxyphenyl)methanol (3h): white solid, yield 96\%, m.p. 71-73 ${ }^{\circ} \mathrm{C} ;{ }^{1} \mathrm{H}$ NMR (400 MHz, DMSO- $\left.d_{6}\right) \delta=3.64(\mathrm{td}, J=6.2 \mathrm{~Hz}, 1.3 \mathrm{~Hz}, 2 \mathrm{H}), 3.77(\mathrm{~d}$, $J=1.3 \mathrm{~Hz}, 6 \mathrm{H}), 4.11(\mathrm{td}, J=6.2 \mathrm{~Hz}, 1.3 \mathrm{~Hz}, 2 \mathrm{H}), 4.44(\mathrm{~d}, J=5.6 \mathrm{~Hz}, 2 \mathrm{H}), 5.16(\mathrm{td}, J=5.7 \mathrm{~Hz}$, $1.3 \mathrm{~Hz}, 1 \mathrm{H}), 6.64(\mathrm{~s}, 2 \mathrm{H}) ;{ }^{13} \mathrm{C}$ NMR $\left(101 \mathrm{MHz}, \mathrm{DMSO}-d_{6}\right) \delta=31.6\left(\mathrm{CH}_{2}\right), 55.9\left(2 \mathrm{CH}_{3}\right)$, $62.9\left(\mathrm{CH}_{2} \mathrm{OH}\right), 72.3\left(\mathrm{CH}_{2}\right), 103.4\left(2 \mathrm{CH}_{\mathrm{Ar}}\right), 134.4\left(\mathrm{C}_{\mathrm{q}}\right), 138.7\left(\mathrm{C}_{\mathrm{q}}\right), 152.6\left(2 \mathrm{C}_{\mathrm{q}}\right)$; HRMS (ESIMS) $\mathrm{m} / \mathrm{z}$ calcd for $\mathrm{C}_{11} \mathrm{H}_{15} \mathrm{BrNaO}_{4}[\mathrm{M}+\mathrm{H}]^{+}$: 291.0232, found: 291.0228; Rf (petroleum ether $/ \mathrm{EtOAc}=2 / 8)$ 0.50, according to [108].

(3-(2-Bromoethoxy)phenyl)methanol (3i): colorless oil, yield 90\%; ${ }^{1} \mathrm{H}$ NMR $(250 \mathrm{MHz}$, DMSO- $\left.d_{6}\right) \delta=3.70-3.87(\mathrm{~m}, 2 \mathrm{H}), 4.16-4.34(\mathrm{~m}, 2 \mathrm{H}), 4.48(\mathrm{~d}, J=5.8 \mathrm{~Hz}, 2 \mathrm{H}), 5.17(\mathrm{t}, J=5.8 \mathrm{~Hz}$, $1 \mathrm{H}), 6.76-6.86(\mathrm{~m}, 1 \mathrm{H}), 6.86-6.96(\mathrm{~m}, 2 \mathrm{H}), 7.24(\mathrm{t}, J=8.0 \mathrm{~Hz}, 1 \mathrm{H}) ;{ }^{13} \mathrm{C} \mathrm{NMR}(101 \mathrm{MHz}$, DMSO-d $\left.d_{6}\right) \delta=31.5\left(\mathrm{CH}_{2}\right), 62.7\left(\mathrm{CH}_{2} \mathrm{OH}\right), 67.6\left(\mathrm{CH}_{2}\right), 112.5\left(\mathrm{CH}_{\mathrm{Ar}}\right), 112.8\left(\mathrm{CH}_{\mathrm{Ar}}\right), 119.0$ $\left(\mathrm{CH}_{\mathrm{Ar}}\right), 129.2\left(\mathrm{CH}_{\mathrm{Ar}}\right), 144.4\left(\mathrm{C}_{\mathrm{q}}\right), 157.9\left(\mathrm{C}_{\mathrm{q}}\right)$; HRMS (ESI-MS) m/z calcd for $\mathrm{C}_{9} \mathrm{H}_{11} \mathrm{BrNaO}_{2}$ $[\mathrm{M}+\mathrm{Na}]^{+}:$252.9840, found: 252.9834; $\mathrm{Rf}$ (petroleum ether $/ \mathrm{EtOAc}=5 / 5$ ) 0.58, according to [109].

(3-(2-Bromoethoxy)-4-methoxyphenyl)methanol (3j): colorless oil, yield 91\%; ${ }^{1} \mathrm{H}$ NMR $\left(250 \mathrm{MHz}, \mathrm{DMSO}-d_{6}\right) \delta=3.75(\mathrm{~s}, 3 \mathrm{H}), 3.63-3.90(\mathrm{~m}, 2 \mathrm{H}), 4.27(\mathrm{dd}, J=6.1 \mathrm{~Hz}, 5.1 \mathrm{~Hz}, 2 \mathrm{H})$, $4.40(\mathrm{~d}, J=5.7 \mathrm{~Hz}, 2 \mathrm{H}), 5.05(\mathrm{t}, J=5.7 \mathrm{~Hz}, 1 \mathrm{H}), 6.84-6.90(\mathrm{~m}, 1 \mathrm{H}), 6.91(\mathrm{~s}, 1 \mathrm{H}), 6.92-6.96$ $(\mathrm{m}, 1 \mathrm{H}) ;{ }^{13} \mathrm{C} \mathrm{NMR}\left(101 \mathrm{MHz}, \mathrm{DMSO}-d_{6}\right) \delta=31.4\left(\mathrm{CH}_{2}\right), 55.7\left(\mathrm{CH}_{3}\right), 62.6\left(\mathrm{CH}_{2} \mathrm{OH}\right), 68.7$ $\left(\mathrm{CH}_{2}\right), 112.2\left(\mathrm{CH}_{\mathrm{Ar}}\right), 112.9\left(\mathrm{CH}_{\mathrm{Ar}}\right), 119.6\left(\mathrm{CH}_{\mathrm{Ar}}\right), 135.2\left(\mathrm{C}_{\mathrm{q}}\right), 147.1\left(\mathrm{C}_{\mathrm{q}}\right), 148.0\left(\mathrm{C}_{\mathrm{q}}\right)$; HRMS (ESI-MS) $\mathrm{m} / \mathrm{z}$ calcd for $\mathrm{C}_{10} \mathrm{H}_{13} \mathrm{BrNaO}_{3}[\mathrm{M}+\mathrm{Na}]^{+}$: 282.9946, found: 282.9937; Rf (petroleum ether $/ \mathrm{EtOAc}=3 / 7) 0.64$.

(3-(2-Bromoethoxy)-4-nitrophenyl)methanol (3k): greenish yellow oil, yield 97\%; ${ }^{1} \mathrm{H} \mathrm{NMR}$ $\left(250 \mathrm{MHz}, \mathrm{DMSO}-d_{6}\right) \delta=3.75-3.86(\mathrm{~m}, 2 \mathrm{H}), 4.44-4.53(\mathrm{~m}, 2 \mathrm{H}), 4.57(\mathrm{~d}, J=5.7 \mathrm{~Hz}, 2 \mathrm{H})$, $5.48(\mathrm{t}, J=5.7 \mathrm{~Hz}, 1 \mathrm{H}), 7.09(\mathrm{ddt}, J=8.3 \mathrm{~Hz}, 1.5 \mathrm{~Hz}, 0.8 \mathrm{~Hz}, 1 \mathrm{H}), 7.23-7.34(\mathrm{~m}, 1 \mathrm{H}), 7.86$ $(\mathrm{d}, J=8.3 \mathrm{~Hz}, 1 \mathrm{H}) ;{ }^{13} \mathrm{C}$ NMR $\left(101 \mathrm{MHz}, \mathrm{DMSO}-d_{6}\right) \delta=30.7\left(\mathrm{CH}_{2}\right), 62.0\left(\mathrm{CH}_{2} \mathrm{OH}\right), 69.2$ $\left(\mathrm{CH}_{2}\right), 112.6\left(\mathrm{CH}_{\mathrm{Ar}}\right), 118.4\left(\mathrm{CH}_{\mathrm{Ar}}\right), 125.1\left(\mathrm{CH}_{\mathrm{Ar}}\right), 138.0\left(\mathrm{C}_{\mathrm{q}}\right), 150.3\left(\mathrm{C}_{\mathrm{q}}\right), 150.7\left(\mathrm{C}_{\mathrm{q}}\right)$; HRMS (ESI-MS) $\mathrm{m} / \mathrm{z}$ calcd for $\mathrm{C}_{9} \mathrm{H}_{11} \mathrm{BrNO}_{4}[\mathrm{M}+\mathrm{H}]^{+}:$275.9871, found: 275.9866; Rf (petroleum ether $/ \mathrm{EtOAc}=3 / 7) 0.54$.

(3-(2-Bromoethoxy)-2-chloro-4-methoxyphenyl)methanol (3l): white solid, yield 99\%, m.p. 76-78 ${ }^{\circ} \mathrm{C} ;{ }^{1} \mathrm{H}$ NMR (400 MHz, DMSO- $\left.d_{6}\right) \delta=3.73(\mathrm{t}, J=5.8 \mathrm{~Hz}, 2 \mathrm{H}), 3.82(\mathrm{~s}, 3 \mathrm{H}), 4.24(\mathrm{t}$, $J=5.8 \mathrm{~Hz}, 2 \mathrm{H}), 4.48(\mathrm{~d}, J=5.0 \mathrm{~Hz}, 2 \mathrm{H}), 5.24(\mathrm{t}, J=5.6 \mathrm{~Hz}, 1 \mathrm{H}), 7.05(\mathrm{~d}, J=8.6 \mathrm{~Hz}, 1 \mathrm{H}), 7.23$ $(\mathrm{t}, J=8.5 \mathrm{~Hz}, 0.9 \mathrm{~Hz}, 1 \mathrm{H}) ;{ }^{13} \mathrm{C}$ NMR $\left(101 \mathrm{MHz}, \mathrm{DMSO}-d_{6}\right) \delta=31.5\left(\mathrm{CH}_{2}\right), 56.1\left(\mathrm{CH}_{3}\right), 60.2$ $\left(\mathrm{CH}_{2} \mathrm{OH}\right), 72.4\left(\mathrm{CH}_{2}\right), 111.2\left(\mathrm{CH}_{\mathrm{Ar}}\right), 123.1\left(\mathrm{CH}_{\mathrm{Ar}}\right), 125.2\left(\mathrm{C}_{\mathrm{q}}\right), 132.3\left(\mathrm{C}_{\mathrm{q}}\right), 142.9\left(\mathrm{C}_{\mathrm{q}}\right), 151.9$ $\left(\mathrm{C}_{\mathrm{q}}\right)$; HRMS (ESI-MS) $\mathrm{m} / \mathrm{z}$ calcd for $\mathrm{C}_{10} \mathrm{H}_{12} \mathrm{BrClNaO}_{3}[\mathrm{M}+\mathrm{Na}]^{+}$: 316.9556, found: 316.9552; $\mathrm{Rf}$ (petroleum ether $/ \mathrm{EtOAc}=3 / 7$ ) 0.66.

(2-Bromo-3-(2-bromoethoxy)-4-methoxyphenyl)methanol (3m): white solid, yield 99\%, m.p. $68-70^{\circ} \mathrm{C} ;{ }^{1} \mathrm{H}$ NMR $\left(250 \mathrm{MHz}, \mathrm{DMSO}-d_{6}\right) \delta=3.75(\mathrm{dd}, J=6.2 \mathrm{~Hz}, 5.6 \mathrm{~Hz}, 2 \mathrm{H}), 3.82(\mathrm{~s}, 3 \mathrm{H})$, $4.23(\mathrm{dd}, J=6.2 \mathrm{~Hz}, 5.6 \mathrm{~Hz}, 2 \mathrm{H}), 4.45(\mathrm{dd}, J=5.6 \mathrm{~Hz}, 0.9 \mathrm{~Hz}, 2 \mathrm{H}), 5.29(\mathrm{t}, J=5.6 \mathrm{~Hz}, 1 \mathrm{H})$, $7.10(\mathrm{~d}, J=8.6 \mathrm{~Hz}, 1 \mathrm{H}), 7.24(\mathrm{dt}, J=8.5 \mathrm{~Hz}, 0.8 \mathrm{~Hz}, 1 \mathrm{H}) ;{ }^{13} \mathrm{C}$ NMR $\left(101 \mathrm{MHz}, \mathrm{DMSO}-d_{6}\right)$ $\delta=31.5\left(\mathrm{CH}_{2}\right), 56.2\left(\mathrm{CH}_{3}\right), 62.5\left(\mathrm{CH}_{2} \mathrm{OH}\right), 72.3\left(\mathrm{CH}_{2}\right), 111.8\left(\mathrm{CH}_{\mathrm{Ar}}\right), 116.4\left(\mathrm{C}_{\mathrm{q}}\right), 123.3\left(\mathrm{CH}_{\mathrm{Ar}}\right)$, $133.7\left(\mathrm{C}_{\mathrm{q}}\right), 143.9\left(\mathrm{C}_{\mathrm{q}}\right), 151.7\left(\mathrm{C}_{\mathrm{q}}\right)$; HRMS (ESI-MS) $\mathrm{m} / \mathrm{z}$ calcd for $\mathrm{C}_{10} \mathrm{H}_{12} \mathrm{Br}_{2} \mathrm{NaO}_{3}[\mathrm{M}+\mathrm{Na}]^{+}$: 360.9051, found: 360.9039; Rf (petroleum ether $/ \mathrm{EtOAc}=3 / 7$ ) 0.63 .

(2-(2-Bromoethoxy)phenyl)methanol (3n): pale yellow oil, yield $88 \% ;{ }^{1} \mathrm{H} \mathrm{NMR}(250 \mathrm{MHz}$, DMSO- $\left.d_{6}\right) \delta=3.72-3.87(\mathrm{~m}, 2 \mathrm{H}), 4.24-4.38(\mathrm{~m}, 2 \mathrm{H}), 4.56(\mathrm{dd}, J=5.5 \mathrm{~Hz}, 0.8 \mathrm{~Hz}, 2 \mathrm{H}), 4.97$ $(\mathrm{t}, J=5.6 \mathrm{~Hz}, 1 \mathrm{H}), 6.88-7.02(\mathrm{~m}, 2 \mathrm{H}), 7.20(\mathrm{dddt}, J=8.1 \mathrm{~Hz}, 7.5 \mathrm{~Hz}, 1.8 \mathrm{~Hz}, 0.6 \mathrm{~Hz}, 1 \mathrm{H})$, 7.40 (dddd, $J=7.4 \mathrm{~Hz}, 1.9 \mathrm{~Hz}, 1.0 \mathrm{~Hz}, 0.6 \mathrm{~Hz}, 1 \mathrm{H} ;{ }^{13} \mathrm{C}$ NMR $\left(101 \mathrm{MHz}, \mathrm{DMSO}-d_{6}\right) \delta=31.8$ 
$\left(\mathrm{CH}_{2}\right), 57.7\left(\mathrm{CH}_{2} \mathrm{OH}\right), 67.9\left(\mathrm{CH}_{2}\right), 111.5\left(\mathrm{CH}_{\mathrm{Ar}}\right), 120.7\left(\mathrm{CH}_{\mathrm{Ar}}\right), 126.9\left(\mathrm{CH}_{\mathrm{Ar}}\right), 127.5\left(\mathrm{CH}_{\mathrm{Ar}}\right)$, $130.9\left(\mathrm{C}_{\mathrm{q}}\right), 154.5\left(\mathrm{C}_{\mathrm{q}}\right)$; HRMS (ESI-MS) $\mathrm{m} / \mathrm{z}$ calcd for $\mathrm{C}_{9} \mathrm{H}_{11} \mathrm{BrNaO}_{2}[\mathrm{M}+\mathrm{Na}]^{+}: 252.9840$, found: 252.9834; Rf (petroleum ether $/ \mathrm{EtOAc}=5 / 5) 0.49$.

(5-Bromo-2-(2-bromoethoxy)phenyl)methanol (3o): pale yellow oil, yield $89 \% ;{ }^{1} \mathrm{H}$ NMR $\left(250 \mathrm{MHz}\right.$, DMSO- $\left.d_{6}\right) \delta=3.74-3.84(\mathrm{~m}, 2 \mathrm{H}), 4.26-4.36(\mathrm{~m}, 2 \mathrm{H}), 4.53(\mathrm{dt}, J=5.67 \mathrm{~Hz}, 0.85 \mathrm{~Hz}$, 2H), $5.19(\mathrm{t}, J=5.6 \mathrm{~Hz}, 1 \mathrm{H}), 6.93(\mathrm{~d}, J=8.6 \mathrm{~Hz}, 1 \mathrm{H}), 7.36(\mathrm{ddt}, J=8.6 \mathrm{~Hz}, 2.6 \mathrm{~Hz}, 0.7 \mathrm{~Hz}$, $1 \mathrm{H}), 7.50(\mathrm{dt}, J=2.6 \mathrm{~Hz}, 0.9 \mathrm{~Hz}, 1 \mathrm{H}) ;{ }^{13} \mathrm{C}$ NMR $\left(101 \mathrm{MHz}, \mathrm{DMSO}-d_{6}\right) \delta=31.5\left(\mathrm{CH}_{2}\right), 57.3$ $\left(\mathrm{CH}_{2} \mathrm{OH}\right), 68.1\left(\mathrm{CH}_{2}\right), 112.5\left(\mathrm{C}_{\mathrm{q}}\right), 113.8\left(\mathrm{CH}_{\mathrm{Ar}}\right), 129.1\left(\mathrm{CH}_{\mathrm{Ar}}\right), 129.8\left(\mathrm{CH}_{\mathrm{Ar}}\right), 133.7\left(\mathrm{C}_{\mathrm{q}}\right), 153.6$ $\left(\mathrm{C}_{\mathrm{q}}\right.$ ); HRMS (ESI-MS) $\mathrm{m} / \mathrm{z}$ calcd for $\mathrm{C}_{9} \mathrm{H}_{10} \mathrm{Br}_{2} \mathrm{NaO}_{2}[\mathrm{M}+\mathrm{Na}]^{+}$: 330.8945, found: 330.8953; $\mathrm{Rf}$ (petroleum ether $/ \mathrm{EtOAc}=5 / 5$ ) 0.47

2-(4-(2-Bromoethoxy)phenyl)ethanol (3p): white needle crystals, yield $82 \%$, m.p. $65-68{ }^{\circ} \mathrm{C}$; ${ }^{1} \mathrm{H}$ NMR $\left(250 \mathrm{MHz}\right.$, DMSO- $\left.d_{6}\right) \delta=2.65(\mathrm{t}, J=7.1 \mathrm{~Hz}, 2 \mathrm{H}), 3.55(\mathrm{td}, J=7.1 \mathrm{~Hz}, 5.2 \mathrm{~Hz}, 2 \mathrm{H})$, $3.72-3.83(\mathrm{~m}, 2 \mathrm{H}), 4.21-4.33(\mathrm{~m}, 2 \mathrm{H}), 4.58(\mathrm{t}, J=5.2 \mathrm{~Hz}, 1 \mathrm{H}), 6.79-6.93(\mathrm{~m}, 2 \mathrm{H}), 7.06-7.23$ $(\mathrm{m}, 2 \mathrm{H}) ;{ }^{13} \mathrm{C}$ NMR $\left(101 \mathrm{MHz}, \mathrm{DMSO}-d_{6}\right) \delta=31.5\left(\mathrm{CH}_{2}\right), 38.1\left(\mathrm{CH}_{2}\right), 62.4\left(\mathrm{CH}_{2} \mathrm{OH}\right), 67.7$ $\left(\mathrm{CH}_{2}\right), 114.4\left(2 \mathrm{CH}_{\mathrm{Ar}}\right), 129.8\left(2 \mathrm{CH}_{\mathrm{Ar}}\right), 132.0\left(\mathrm{C}_{\mathrm{q}}\right), 156.2\left(\mathrm{C}_{\mathrm{q}}\right)$; HRMS (ESI-MS) $\mathrm{m} / \mathrm{z}$ calcd for $\mathrm{C}_{10} \mathrm{H}_{14} \mathrm{BrO}_{2}[\mathrm{M}+\mathrm{H}]^{+}: 245.0177$, found: 245.0171; Rf (petroleum ether $/$ EtOAc $=3 / 7$ ) 0.60 .

\subsubsection{General Procedure for the Synthesis of the (hydroxyalkyl)phenoxy Nitrates (4a-p)}

To a solution of the appropriate (bromoethoxy)aromatic alcohol derivatives 3a-p (1.0 Eq.), in acetonitrile ( $50 \mathrm{~mL}$ for $10 \mathrm{mmol}$ scale), silver nitrate (1.5 Eq.) was added. The mixture was stirred under reflux and darkness for approximately $12 \mathrm{~h}$, according to the methods reported in the literature [110-117], which were adapted to our synthesis in terms of the ratio of reagents, solvent, time of reaction, purification method. At the end of the reaction, brine was added to precipitate the excess of silver nitrate. After filtration through celite, the mixture was extracted with diethyl ether $(2 \times 50 \mathrm{~mL})$. The combined organic layers were washed with distilled water $(50 \mathrm{~mL})$ and brine $(50 \mathrm{~mL})$, dried over anhydrous magnesium sulfate, filtered and concentrated under reduced pressure. The crude products were purified by flash chromatography (silicagel, petroleum ether/EtOAc) to obtain the pure products $4 \mathbf{a}-\mathbf{p}$.

2-(4-(Hydroxymethyl)phenoxy)ethyl nitrate (4a): white solid, yield $97 \%$, m.p. $55-57^{\circ} \mathrm{C}$; ${ }^{1} \mathrm{H}$ NMR $\left(250 \mathrm{MHz}\right.$, DMSO- $\left.d_{6}\right) \delta=4.23-4.34(\mathrm{~m}, 2 \mathrm{H}), 4.42(\mathrm{~d}, J=5.6 \mathrm{~Hz}, 2 \mathrm{H}), 4.81-4.91$ $(\mathrm{m}, 2 \mathrm{H}), 5.05(\mathrm{t}, J=5.6 \mathrm{~Hz}, 1 \mathrm{H}), 6.79-6.98(\mathrm{~m}, 2 \mathrm{H}), 7.15-7.31(\mathrm{~m}, 2 \mathrm{H}) ;{ }^{13} \mathrm{C} \mathrm{NMR}(101 \mathrm{MHz}$, DMSO- $\left.d_{6}\right) \delta=62.5\left(\mathrm{CH}_{2} \mathrm{OH}\right), 64.0\left(\mathrm{CH}_{2}\right), 72.1\left(\mathrm{CH}_{2}\right), 114.1\left(2 \mathrm{CH}_{\mathrm{Ar}}\right), 127.9\left(2 \mathrm{CH}_{\mathrm{Ar}}\right), 135.2$ $\left(\mathrm{C}_{\mathrm{q}}\right), 156.7\left(\mathrm{C}_{\mathrm{q}}\right)$; HRMS (ESI-MS) $\mathrm{m} / \mathrm{z}$ calcd for $\mathrm{C}_{9} \mathrm{H}_{11} \mathrm{NNaO}_{5}[\mathrm{M}+\mathrm{Na}]^{+}: 236.0535$, found: 236.0526; Rf (petroleum ether $/ \mathrm{EtOAc}=6 / 4$ ) 0.62 .

2-(2-Fluoro-4-(hydroxymethyl)phenoxy)ethyl nitrate ( $4 \boldsymbol{b})$ : greenish yellow oil, yield $95 \%$; ${ }^{1} \mathrm{H}$ NMR $\left(400 \mathrm{MHz}\right.$, DMSO- $\left.d_{6}\right) \delta=4.33-4.39(\mathrm{~m}, 2 \mathrm{H}), 4.42(\mathrm{~d}, J=5.6 \mathrm{~Hz}, 2 \mathrm{H}), 4.84-4.94$ $(\mathrm{m}, 2 \mathrm{H}), 5.19(\mathrm{t}, J=5.7 \mathrm{~Hz}, 1 \mathrm{H}), 7.03-7.09(\mathrm{~m}, 1 \mathrm{H}), 7.11-7.18(\mathrm{~m}, 2 \mathrm{H}) ;{ }^{13} \mathrm{C} \mathrm{NMR}(101 \mathrm{MHz}$, DMSO- $\left.d_{6}\right) \delta=61.9\left(\mathrm{CH}_{2} \mathrm{OH}\right), 65.4\left(\mathrm{CH}_{2}\right), 71.9\left(\mathrm{CH}_{2}\right), 114.2\left(\mathrm{~d}, J=18.0 \mathrm{~Hz}, \mathrm{CH}_{\mathrm{Ar}}\right), 115.1$ $\left(\mathrm{d}, J=1.8 \mathrm{~Hz}, \mathrm{CH}_{\mathrm{Ar}}\right), 122.4\left(\mathrm{~d}, J=3.5 \mathrm{~Hz}, \mathrm{CH}_{\mathrm{Ar}}\right), 136.7\left(\mathrm{~d}, J=5.5 \mathrm{~Hz}, \mathrm{C}_{\mathrm{q}}\right), 144.2(\mathrm{~d}$, $\left.J=10.7 \mathrm{~Hz}, \mathrm{C}_{\mathrm{q}}\right), 151.5\left(\mathrm{~d}, J=243.9 \mathrm{~Hz}, \mathrm{C}_{\mathrm{q}} \mathrm{F}\right) ;{ }^{19} \mathrm{~F}$ NMR $\left(376 \mathrm{MHz}, \mathrm{DMSO}-d_{6}\right) \delta=-134.94$ $\left(\mathrm{dd}, J=12.1 \mathrm{~Hz}, 8.3 \mathrm{~Hz}\right.$ ); HR.MS (ESI-MS) $\mathrm{m} / \mathrm{z}$ calcd for $\mathrm{C}_{9} \mathrm{H}_{10} \mathrm{FNNaO}_{5}[\mathrm{M}+\mathrm{Na}]^{+}:$: 254.0441, found: 254.0436; $\mathrm{Rf}\left(\mathrm{CH}_{2} \mathrm{Cl}_{2} / \mathrm{CH}_{3} \mathrm{OH}=9.8 / 0.2\right) 0.66$.

2-(2-Chloro-4-(hydroxymethyl)phenoxy)ethyl nitrate (4c): orange oil, yield $97 \% ;{ }^{1} \mathrm{H}$ NMR $\left(400 \mathrm{MHz}, \mathrm{DMSO}-d_{6}\right) \delta=4.33-4.39(\mathrm{~m}, 2 \mathrm{H}), 4.42(\mathrm{~d}, J=5.8 \mathrm{~Hz}, 2 \mathrm{H}), 4.85-4.95(\mathrm{~m}, 2 \mathrm{H}), 5.20$ $(\mathrm{t}, J=5.8 \mathrm{~Hz}, 1 \mathrm{H}), 7.14(\mathrm{~d}, J=8.4 \mathrm{~Hz}, 1 \mathrm{H}), 7.23(\mathrm{dd}, J=8.4 \mathrm{~Hz}, 2.0 \mathrm{~Hz}, 1 \mathrm{H}), 7.36(\mathrm{~d}, J=2.0 \mathrm{~Hz}$, $1 \mathrm{H}) ;{ }^{13} \mathrm{C}$ NMR $\left(101 \mathrm{MHz}\right.$, DMSO- $\left.d_{6}\right) \delta=61.8\left(\mathrm{CH}_{2} \mathrm{OH}\right), 65.4\left(\mathrm{CH}_{2}\right), 71.7\left(\mathrm{CH}_{2}\right), 114.0\left(\mathrm{CH}_{\mathrm{Ar}}\right)$, $121.2\left(\mathrm{C}_{\mathrm{q}}\right), 126.3\left(\mathrm{CH}_{\mathrm{Ar}}\right), 128.1\left(\mathrm{CH}_{\mathrm{Ar}}\right), 136.7\left(\mathrm{C}_{\mathrm{q}}\right), 151.9\left(\mathrm{C}_{\mathrm{q}}\right)$;HRMS (ESI-MS) $\mathrm{m} / \mathrm{z}$ calcd for $\mathrm{C}_{9} \mathrm{H}_{10} \mathrm{ClNNaO}_{5}[\mathrm{M}+\mathrm{Na}]^{+}: 270.0145$, found: $270.0140 ; \mathrm{Rf}\left(\mathrm{CH}_{2} \mathrm{Cl}_{2} / \mathrm{CH}_{3} \mathrm{OH}=9.8 / 0.2\right) 0.63$.

2-(2,6-Dichloro-4-(hydroxymethyl)phenoxy)ethyl nitrate (4d): greenish yellow oil, yield 99\%; ${ }^{1} \mathrm{H}$ NMR $\left(250 \mathrm{MHz}, \mathrm{DMSO}-d_{6}\right) \delta=4.20-4.34(\mathrm{~m}, 2 \mathrm{H}), 4.46(\mathrm{dt}, J=5.8 \mathrm{~Hz}, 0.8 \mathrm{~Hz}$, $2 \mathrm{H}), 4.78-4.94(\mathrm{~m}, 2 \mathrm{H}), 5.40(\mathrm{t}, J=5.8 \mathrm{~Hz}, 1 \mathrm{H}), 7.41(\mathrm{t}, J=0.8 \mathrm{~Hz} 2 \mathrm{H}) ;{ }^{13} \mathrm{C} \mathrm{NMR}(101 \mathrm{MHz}$, DMSO- $\left.d_{6}\right) \delta=61.2\left(\mathrm{CH}_{2} \mathrm{OH}\right), 69.4\left(\mathrm{CH}_{2}\right), 72.5\left(\mathrm{CH}_{2}\right), 126.8\left(2 \mathrm{CH}_{\mathrm{Ar}}\right), 127.9\left(2 \mathrm{C}_{\mathrm{q}}\right), 141.4$ 
$\left(\mathrm{C}_{\mathrm{q}}\right)$, $148.4\left(\mathrm{C}_{\mathrm{q}}\right)$; HRMS (ESI-MS) $\mathrm{m} / \mathrm{z}$ calcd for $\mathrm{C}_{9} \mathrm{H}_{9} \mathrm{Cl}_{2} \mathrm{NNaO}_{5}[\mathrm{M}+\mathrm{Na}]^{+}: 303.9755$, found: 303.9747; $\mathrm{Rf}\left(\mathrm{CH}_{2} \mathrm{Cl}_{2} / \mathrm{CH}_{3} \mathrm{OH}=9.8 / 0.2\right) 0.53$.

2-(4-(Hydroxymethyl)-2-nitrophenoxy)ethyl nitrate (4e): brown greenish yellow solid, yield $95 \%$, m.p. $56-58^{\circ} \mathrm{C} ;{ }^{1} \mathrm{H}$ NMR $\left(400 \mathrm{MHz}\right.$, DMSO- $\left.d_{6}\right) \delta=4.44-4.53(\mathrm{~m}, 4 \mathrm{H}), 4.86-4.93$ $(\mathrm{m}, 2 \mathrm{H}), 5.36(\mathrm{t}, J=5.7 \mathrm{~Hz}, 1 \mathrm{H}), 7.36(\mathrm{~d}, J=8.6 \mathrm{~Hz}, 1 \mathrm{H}), 7.59(\mathrm{dd}, J=8.6 \mathrm{~Hz}, 2.1 \mathrm{~Hz}, 1 \mathrm{H})$, $7.80(\mathrm{~d}, J=2.1 \mathrm{~Hz}, 1 \mathrm{H}) ;{ }^{13} \mathrm{C}$ NMR $\left(101 \mathrm{MHz}, \mathrm{DMSO}-d_{6}\right) \delta=61.4\left(\mathrm{CH}_{2} \mathrm{OH}\right), 66.1\left(\mathrm{CH}_{2}\right)$, $71.4\left(\mathrm{CH}_{2}\right), 115.4\left(\mathrm{CH}_{\mathrm{Ar}}\right), 122.7\left(\mathrm{CH}_{\mathrm{Ar}}\right), 132.3\left(\mathrm{CH}_{\mathrm{Ar}}\right), 136.0\left(\mathrm{C}_{\mathrm{q}}\right), 139.3\left(\mathrm{C}_{\mathrm{q}}\right), 149.3\left(\mathrm{C}_{\mathrm{q}}\right)$; HRMS (ESI-MS) m/z calcd for $\mathrm{C}_{9} \mathrm{H}_{10} \mathrm{~N}_{2} \mathrm{NaO}_{7}$ [M+Na] $]^{+}$: 281.0386, found: 281.0381; Rf $\left(\mathrm{CH}_{2} \mathrm{Cl}_{2} / \mathrm{CH}_{3} \mathrm{OH}=9.8 / 0.2\right) 0.52$.

2-(4-(hydroxymethyl)-2-methoxyphenoxy)ethyl nitrate (4f): brown solid, yield 99\%, m.p. $51-52{ }^{\circ} \mathrm{C} ;{ }^{1} \mathrm{H}$ NMR $\left(250 \mathrm{MHz}, \mathrm{DMSO}-d_{6}\right) \delta=3.75(\mathrm{~s}, 3 \mathrm{H}), 4.17-4.31(\mathrm{~m}, 2 \mathrm{H}), 4.42(\mathrm{~d}$, $J=5.5 \mathrm{~Hz}, 2 \mathrm{H}), 4.78-4.91(\mathrm{~m}, 2 \mathrm{H}), 5.09(\mathrm{t}, J=5.6 \mathrm{~Hz}, 1 \mathrm{H}), 6.81(\mathrm{ddt}, J=8.0 \mathrm{~Hz}, 1.9 \mathrm{~Hz}, 0.7 \mathrm{~Hz}$, $1 \mathrm{H}), 6.87-7.00(\mathrm{~m}, 2 \mathrm{H}) ;{ }^{13} \mathrm{C}$ NMR $\left(101 \mathrm{MHz}\right.$, DMSO- $\left.d_{6}\right) \delta=55.4\left(\mathrm{CH}_{3}\right), 62.7\left(\mathrm{CH}_{2} \mathrm{OH}\right), 65.2$ $\left(\mathrm{CH}_{2}\right), 72.2\left(\mathrm{CH}_{2}\right), 110.9\left(\mathrm{CH}_{\mathrm{Ar}}\right), 114.0\left(\mathrm{CH}_{\mathrm{Ar}}\right), 118.5\left(\mathrm{CH}_{\mathrm{Ar}}\right), 136.4\left(\mathrm{C}_{\mathrm{q}}\right), 146.0\left(\mathrm{C}_{\mathrm{q}}\right), 149.0$ $\left(\mathrm{C}_{\mathrm{q}}\right)$; HRMS (ESI-MS) $\mathrm{m} / \mathrm{z}$ calcd for $\mathrm{C}_{10} \mathrm{H}_{13} \mathrm{NNaO}_{6}[\mathrm{M}+\mathrm{Na}]^{+}: 266.0641$, found: 266.0632; $\mathrm{Rf}$ (petroleum ether $/ \mathrm{EtOAc}=6 / 4$ ) 0.55 .

2-(2-ethoxy-4-(hydroxymethyl)phenoxy)ethyl nitrate (4g): greenish yellow solid, yield $99 \%$, m.p. 57-59 ${ }^{\circ} \mathrm{C} ;{ }^{1} \mathrm{H}$ NMR $\left(250 \mathrm{MHz}, \mathrm{DMSO}-d_{6}\right) \delta=1.31(\mathrm{t}, J=6.98 \mathrm{~Hz}, 3 \mathrm{H}), 4.01$ $(\mathrm{q}, J=6.96 \mathrm{~Hz}, 2 \mathrm{H}), 4.17-4.29(\mathrm{~m}, 2 \mathrm{H}), 4.41(\mathrm{~d}, J=5.1 \mathrm{~Hz}, 2 \mathrm{H}), 4.76-4.91(\mathrm{~m}, 2 \mathrm{H}), 5.08(\mathrm{t}$, $J=5.6 \mathrm{~Hz}, 1 \mathrm{H}), 6.81(\mathrm{ddt}, J=8.1 \mathrm{~Hz}, 1.9 \mathrm{~Hz}, 0.7 \mathrm{~Hz}, 1 \mathrm{H}), 6.94(\mathrm{~d}, J=8.1 \mathrm{~Hz}, 1 \mathrm{H}), 6.94$ $(\mathrm{d}, J=1.9 \mathrm{~Hz}, 1 \mathrm{H}) ;{ }^{13} \mathrm{C}$ NMR $\left(101 \mathrm{MHz}, \mathrm{DMSO}-d_{6}\right) \delta=14.7\left(\mathrm{CH}_{3}\right), 62.7\left(\mathrm{CH}_{2} \mathrm{OH}\right), 63.8$ $\left(\mathrm{CH}_{2}\right), 65.7\left(\mathrm{CH}_{2}\right), 72.2\left(\mathrm{CH}_{2}\right), 112.4\left(\mathrm{CH}_{\mathrm{Ar}}\right), 114.9\left(\mathrm{CH}_{\mathrm{Ar}}\right), 118.7\left(\mathrm{CH}_{\mathrm{Ar}}\right), 136.6\left(\mathrm{C}_{\mathrm{q}}\right), 146.3$ $\left(\mathrm{C}_{\mathrm{q}}\right), 148.5\left(\mathrm{C}_{\mathrm{q}}\right)$; HRMS (ESI-MS) $\mathrm{m} / \mathrm{z}$ calcd for $\mathrm{C}_{11} \mathrm{H}_{15} \mathrm{NNaO}_{6}[\mathrm{M}+\mathrm{Na}]^{+}: 280.0797$, found: 280.0794; Rf (petroleum ether $/ \mathrm{EtOAc}=6 / 4$ ) 0.48 .

2-(4-(Hydroxymethyl)-2,6-dimethoxyphenoxy)ethyl nitrate (4h): greenish yellow solid, yield $99 \%$, m.p. $56-58{ }^{\circ} \mathrm{C} ;{ }^{1} \mathrm{H}$ NMR $\left(250 \mathrm{MHz}\right.$, DMSO- $\left.d_{6}\right) \delta=3.74(\mathrm{~s}, 6 \mathrm{H}), 4.04-4.15(\mathrm{~m}$, $2 \mathrm{H}), 4.43(\mathrm{dt}, J=5.8 \mathrm{~Hz}, 0.6 \mathrm{~Hz}, 2 \mathrm{H}), 4.67-4.78(\mathrm{~m}, 2 \mathrm{H}), 5.17(\mathrm{t}, J=5.8 \mathrm{~Hz}, 1 \mathrm{H}), 6.63(\mathrm{~d}$, $J=0.6 \mathrm{~Hz}, 2 \mathrm{H}){ }_{1}^{13} \mathrm{C}$ NMR $\left(101 \mathrm{MHz}, \mathrm{DMSO}-d_{6}\right) \delta=55.8\left(2 \mathrm{CH}_{3}\right), 62.9\left(\mathrm{CH}_{2} \mathrm{OH}\right), 68.4\left(\mathrm{CH}_{2}\right)$, $72.9\left(\mathrm{CH}_{2}\right), 103.3\left(2 \mathrm{CH}_{\mathrm{Ar}}\right), 134.2\left(\mathrm{C}_{\mathrm{q}}\right), 138.9\left(\mathrm{C}_{\mathrm{q}}\right), 152.6\left(2 \mathrm{C}_{\mathrm{q}}\right)$; HRMS (ESI-MS) $\mathrm{m} / \mathrm{z}$ calcd for $\mathrm{C}_{11} \mathrm{H}_{16} \mathrm{NO}_{7}[\mathrm{M}+\mathrm{H}]^{+}: 274.0927$, found: 274.0922; $\mathrm{Rf}$ (petroleum ether $/ \mathrm{EtOAc}=7 / 3$ ) 0.43 .

2-(3-(Hydroxymethyl)phenoxy)ethyl nitrate (4i): colorless oil, yield $89 \% ;{ }^{1} \mathrm{H}$ NMR $\left(250 \mathrm{MHz}, \mathrm{DMSO}-d_{6}\right) \delta=4.16-4.34(\mathrm{~m}, 2 \mathrm{H}), 4.48(\mathrm{~d}, J=5.8 \mathrm{~Hz}, 2 \mathrm{H}), 4.81-4.90(\mathrm{~m}, 2 \mathrm{H})$, $5.17(\mathrm{t}, J=5.8 \mathrm{~Hz}, 1 \mathrm{H}), 6.76-6.86(\mathrm{~m}, 1 \mathrm{H}), 6.86-6.96(\mathrm{~m}, 2 \mathrm{H}), 7.24(\mathrm{t}, J=8.0 \mathrm{~Hz}, 1 \mathrm{H}) ;{ }^{13} \mathrm{C}$ NMR $\left(101 \mathrm{MHz}\right.$, DMSO- $\left.d_{6}\right) \delta=62.8\left(\mathrm{CH}_{2} \mathrm{OH}\right), 67.6\left(\mathrm{CH}_{2}\right), 72.1\left(\mathrm{CH}_{2}\right), 112.5\left(\mathrm{CH}_{\mathrm{Ar}}\right), 112.8$ $\left(\mathrm{CH}_{\mathrm{Ar}}\right), 119.0\left(\mathrm{CH}_{\mathrm{Ar}}\right), 129.2\left(\mathrm{CH}_{\mathrm{Ar}}\right), 144.4\left(\mathrm{C}_{\mathrm{q}}\right), 157.9\left(\mathrm{C}_{\mathrm{q}}\right)$; HRMS (ESI-MS) $\mathrm{m} / \mathrm{z}$ calcd for $\mathrm{C}_{9} \mathrm{H}_{11} \mathrm{NNaO}_{5}[\mathrm{M}+\mathrm{Na}]^{+}:$236.0535, found: 236.0529; $\mathrm{Rf}\left(\mathrm{CH}_{2} \mathrm{Cl}_{2} / \mathrm{CH}_{3} \mathrm{OH}=9.6 / 0.4\right) 0.39$.

2-(5-(Hydroxymethyl)-2-methoxyphenoxy)ethyl nitrate (4j): yellow solid, yield $80 \%$, m.p. $51-53{ }^{\circ} \mathrm{C} ;{ }^{1} \mathrm{H}$ NMR $\left(250 \mathrm{MHz}, \mathrm{DMSO}-d_{6}\right) \delta=3.74(\mathrm{~s}, 3 \mathrm{H}), 4.21-4.30(\mathrm{~m}, 2 \mathrm{H}), 4.41(\mathrm{~d}$, $J=5.7 \mathrm{~Hz}, 2 \mathrm{H}), 4.81-4.90(\mathrm{~m}, 2 \mathrm{H}), 5.06(\mathrm{t}, J=5.7 \mathrm{~Hz}, 1 \mathrm{H}), 6.82-6.98(\mathrm{~m}, 3 \mathrm{H}) ;{ }^{13} \mathrm{C} \mathrm{NMR}$ $\left(101 \mathrm{MHz}, \mathrm{DMSO}-d_{6}\right) \delta=55.6\left(\mathrm{CH}_{3}\right), 62.6\left(\mathrm{CH}_{2} \mathrm{OH}\right), 65.0\left(\mathrm{CH}_{2}\right), 72.1\left(\mathrm{CH}_{2}\right), 112.0\left(\mathrm{CH}_{\mathrm{Ar}}\right)$, $112.8\left(\mathrm{CH}_{\mathrm{Ar}}\right), 119.7\left(\mathrm{CH}_{\mathrm{Ar}}\right), 135.1\left(\mathrm{C}_{\mathrm{q}}\right), 147.0\left(\mathrm{C}_{\mathrm{q}}\right), 148.0\left(\mathrm{C}_{\mathrm{q}}\right)$; HRMS (ESI-MS) m/z calcd for $\mathrm{C}_{10} \mathrm{H}_{13} \mathrm{NNaO}_{6}[\mathrm{M}+\mathrm{Na}]^{+}:$: 266.0641, found: 266.0635; Rf (petroleum ether $/ \mathrm{EtOAc}=7 / 3$ ) 0.63 . 2-(5-(Hydroxymethyl)-2-nitrophenoxy)ethyl nitrate (4k): brown oil, yield $94 \% ;{ }^{1} \mathrm{H}$ NMR $\left(250 \mathrm{MHz}, \mathrm{DMSO}-d_{6}\right) \delta=4.43-4.53(\mathrm{~m}, 2 \mathrm{H}), 4.58(\mathrm{~d}, J=5.7 \mathrm{~Hz}, 2 \mathrm{H}), 4.85-4.96(\mathrm{~m}, 2 \mathrm{H})$, $5.49(\mathrm{t}, J=5.7 \mathrm{~Hz}, 1 \mathrm{H}), 7.10(\mathrm{ddt}, J=8.3 \mathrm{~Hz}, 1.5 \mathrm{~Hz}, 0.7 \mathrm{~Hz}, 1 \mathrm{H}), 7.28-7.33(\mathrm{~m}, 1 \mathrm{H})$, $7.87(\mathrm{~d}, J=8.3 \mathrm{~Hz}, 1 \mathrm{H}) ;{ }^{13} \mathrm{C}$ NMR $\left(101 \mathrm{MHz}, \mathrm{DMSO}-d_{6}\right) \delta=62.0\left(\mathrm{CH}_{2} \mathrm{OH}\right), 65.9\left(\mathrm{CH}_{2}\right)$, $71.3\left(\mathrm{CH}_{2}\right), 112.5\left(\mathrm{CH}_{\mathrm{Ar}}\right), 118.5\left(\mathrm{CH}_{\mathrm{Ar}}\right), 125.1\left(\mathrm{CH}_{\mathrm{Ar}}\right), 137.9\left(\mathrm{C}_{\mathrm{q}}\right), 150.4\left(\mathrm{C}_{\mathrm{q}}\right), 150.7\left(\mathrm{C}_{\mathrm{q}}\right)$; HRMS (ESI-MS) m/z calcd for $\mathrm{C}_{9} \mathrm{H}_{10} \mathrm{~N}_{2} \mathrm{NaO}_{7}[\mathrm{M}+\mathrm{Na}]^{+}$: 281.0386, found: 281.0380; $\mathrm{Rf}$ $\left(\mathrm{CH}_{2} \mathrm{Cl}_{2} / \mathrm{CH}_{3} \mathrm{OH}=9.6 / 0.4\right) 0.53$.

2-(2-Chloro-3-(hydroxymethyl)-6-methoxyphenoxy)ethyl nitrate (4l): white solid, yield 91\%, m.p. 57-59 ${ }^{\circ} \mathrm{C} ;{ }^{1} \mathrm{H}$ NMR $\left(400 \mathrm{MHz}\right.$, DMSO- $\left.d_{6}\right) \delta=3.80(\mathrm{~s}, 3 \mathrm{H}), 4.15-4.29(\mathrm{~m}, 2 \mathrm{H}), 4.48(\mathrm{dd}$, $J=5.6 \mathrm{~Hz}, 0.8 \mathrm{~Hz}, 2 \mathrm{H}), 4.75-4.90(\mathrm{~m}, 2 \mathrm{H}), 5.25(\mathrm{t}, J=5.6 \mathrm{~Hz}, 1 \mathrm{H}), 7.06(\mathrm{~d}, J=8.6 \mathrm{~Hz}, 1 \mathrm{H}), 7.24$ $(\mathrm{dt}, J=8.7 \mathrm{~Hz}, 0.9 \mathrm{~Hz}, 1 \mathrm{H}) ;{ }^{13} \mathrm{C}$ NMR $\left(101 \mathrm{MHz}, \mathrm{DMSO}-d_{6}\right) \delta=56.0\left(\mathrm{CH}_{3}\right), 60.2\left(\mathrm{CH}_{2} \mathrm{OH}\right)$, $68.7\left(\mathrm{CH}_{2}\right), 72.7\left(\mathrm{CH}_{2}\right), 111.1\left(\mathrm{CH}_{\mathrm{Ar}}\right), 123.2\left(\mathrm{CH}_{\mathrm{Ar}}\right), 125.2\left(\mathrm{C}_{\mathrm{q}}\right), 132.3\left(\mathrm{C}_{\mathrm{q}}\right), 142.8\left(\mathrm{C}_{\mathrm{q}}\right), 151.9$ 
$\left(\mathrm{C}_{\mathrm{q}}\right.$ ); HRMS (ESI-MS) $\mathrm{m} / \mathrm{z}$ calcd for $\mathrm{C}_{10} \mathrm{H}_{12} \mathrm{ClNNaO}_{6}[\mathrm{M}+\mathrm{Na}]^{+}$: 300.0251, found: 300.0246; $\operatorname{Rf}\left(\mathrm{CH}_{2} \mathrm{Cl}_{2} / \mathrm{CH}_{3} \mathrm{OH}=9.6 / 0.4\right) 0.55$.

2-(2-Bromo-3-(hydroxymethyl)-6-methoxyphenoxy)ethyl nitrate (4m): white solid, yield $92 \%$, m.p. $58-60{ }^{\circ} \mathrm{C} ;{ }^{1} \mathrm{H}$ NMR $\left(400 \mathrm{MHz}\right.$, DMSO- $\left.d_{6}\right) \delta=3.81(\mathrm{~s}, 3 \mathrm{H}), 4.16-4.25(\mathrm{~m}, 2 \mathrm{H}), 4.44$ $(\mathrm{dd}, J=5.6 \mathrm{~Hz}, 0.8 \mathrm{~Hz}, 2 \mathrm{H}), 4.76-4.87(\mathrm{~m}, 2 \mathrm{H}), 5.29(\mathrm{t}, J=5.6 \mathrm{~Hz}, 1 \mathrm{H}), 7.10(\mathrm{~d}, J=8.5 \mathrm{~Hz}$, $1 \mathrm{H}), 7.25(\mathrm{dt}, J=8.5 \mathrm{~Hz}, 0.8 \mathrm{~Hz}, 1 \mathrm{H}) ;{ }^{13} \mathrm{C}$ NMR $\left(101 \mathrm{MHz}, \mathrm{DMSO}-d_{6}\right) \delta=56.0\left(\mathrm{CH}_{3}\right), 62.5$ $\left(\mathrm{CH}_{2} \mathrm{OH}\right), 68.6\left(\mathrm{CH}_{2}\right), 72.7\left(\mathrm{CH}_{2}\right), 111.8\left(\mathrm{CH}_{\mathrm{Ar}}\right), 116.4\left(\mathrm{C}_{\mathrm{q}}\right), 123.4\left(\mathrm{CH}_{\mathrm{Ar}}\right), 133.7\left(\mathrm{C}_{\mathrm{q}}\right), 143.8$ $\left(\mathrm{C}_{\mathrm{q}}\right), 151.7\left(\mathrm{C}_{\mathrm{q}}\right)$; HRMS (ESI-MS) $\mathrm{m} / \mathrm{z}$ calcd for $\mathrm{C}_{10} \mathrm{H}_{12} \mathrm{BrNNaO}_{6}[\mathrm{M}+\mathrm{Na}]^{+}: 343.9746$, found: 343.9737; $\mathrm{Rf}\left(\mathrm{CH}_{2} \mathrm{Cl}_{2} / \mathrm{CH}_{3} \mathrm{OH}=9.6 / 0.4\right) 0.56$.

2-(2-(Hydroxymethyl)phenoxy)ethyl nitrate $(4 n)$ : orange oil, yield $93 \% ;{ }^{1} \mathrm{H}$ NMR $(400 \mathrm{MHz}$, DMSO- $\left.d_{6}\right) \delta=4.25-4.31(\mathrm{~m}, 2 \mathrm{H}), 4.47(\mathrm{~d}, J=5.6 \mathrm{~Hz}, 2 \mathrm{H}), 4.86-4.92(\mathrm{~m}, 2 \mathrm{H}), 4.98(\mathrm{t}, J=5.6 \mathrm{~Hz}$, $1 \mathrm{H}), 6.97(\mathrm{t}, J=7.4 \mathrm{~Hz}, 2 \mathrm{H}), 7.21(\mathrm{td}, J=7.8 \mathrm{~Hz}, 1.8 \mathrm{~Hz}, 1 \mathrm{H}), 7.39(\mathrm{dd}, J=7.1 \mathrm{~Hz}, 1.2 \mathrm{~Hz}, 1 \mathrm{H})$; ${ }^{13} \mathrm{C}$ NMR $\left(101 \mathrm{MHz}\right.$, DMSO- $\left.d_{6}\right) \delta=57.6\left(\mathrm{CH}_{2} \mathrm{OH}\right), 64.4\left(\mathrm{CH}_{2}\right), 72.0\left(\mathrm{CH}_{2}\right), 111.4\left(\mathrm{CH}_{\mathrm{Ar}}\right), 120.8$ $\left(\mathrm{CH}_{\mathrm{Ar}}\right), 127.0\left(\mathrm{CH}_{\mathrm{Ar}}\right), 127.5\left(\mathrm{CH}_{\mathrm{Ar}}\right), 130.7\left(\mathrm{C}_{\mathrm{q}}\right), 154.5\left(\mathrm{C}_{\mathrm{q}}\right) ; \mathrm{HRMS}$ (ESI-MS) $\mathrm{m} / \mathrm{z}$ calcd for $\mathrm{C}_{9} \mathrm{H}_{11} \mathrm{NNaO}_{5}[\mathrm{M}+\mathrm{Na}]^{+}: 236.0535$, found: 236.0530; $\mathrm{Rf}\left(\mathrm{CH}_{2} \mathrm{Cl}_{2} / \mathrm{CH}_{3} \mathrm{OH}=9.6 / 0.4\right) 0.62$.

2-(4-Bromo-2-(hydroxymethyl)phenoxy)ethyl nitrate (4o): greenish yellow oil, yield 95\%; ${ }^{1} \mathrm{H}$ NMR $\left(250 \mathrm{MHz}, \mathrm{DMSO}-d_{6}\right) \delta=4.26-4.34(\mathrm{~m}, 2 \mathrm{H}), 4.45(\mathrm{dt}, J=5.6 \mathrm{~Hz}, 0.8 \mathrm{~Hz}, 2 \mathrm{H})$, $4.85-4.92(\mathrm{~m}, 2 \mathrm{H}), 5.19(\mathrm{t}, J=5.6 \mathrm{~Hz}, 1 \mathrm{H}), 6.95(\mathrm{~d}, J=8.7 \mathrm{~Hz}, 1 \mathrm{H}), 7.38(\mathrm{ddt}, J=8.7 \mathrm{~Hz}$, $2.6 \mathrm{~Hz}, 0.7 \mathrm{~Hz}, 1 \mathrm{H}), 7.49(\mathrm{dt}, J=2.6 \mathrm{~Hz}, 0.9 \mathrm{~Hz}, 1 \mathrm{H}) ;{ }^{13} \mathrm{C}$ NMR $\left(101 \mathrm{MHz}\right.$, DMSO- $\left.d_{6}\right) \delta=57.2$ $\left(\mathrm{CH}_{2} \mathrm{OH}\right), 64.8\left(\mathrm{CH}_{2}\right), 71.8\left(\mathrm{CH}_{2}\right), 112.6\left(\mathrm{C}_{\mathrm{q}}\right), 113.7\left(\mathrm{CH}_{\mathrm{Ar}}\right), 129.2\left(\mathrm{CH}_{\mathrm{Ar}}\right), 129.9\left(\mathrm{CH}_{\mathrm{Ar}}\right)$, $133.6\left(\mathrm{C}_{\mathrm{q}}\right), 153.7\left(\mathrm{C}_{\mathrm{q}}\right)$; HRMS (ESI-MS) $\mathrm{m} / \mathrm{z}$ calcd for $\mathrm{C}_{9} \mathrm{H}_{10} \mathrm{BrNNaO}_{5}[\mathrm{M}+\mathrm{Na}]^{+}$: 313.9640, found: 313.9634; $\mathrm{Rf}\left(\mathrm{CH}_{2} \mathrm{Cl}_{2} / \mathrm{CH}_{3} \mathrm{OH}=9.6 / 0.4\right) 0.55$.

2-(4-(2-Hydroxyethyl)phenoxy)ethyl nitrate (4p): white solid, yield $98 \%$, m.p. $53-55^{\circ} \mathrm{C}$; ${ }^{1} \mathrm{H}$ NMR $\left(250 \mathrm{MHz}\right.$, DMSO- $\left.d_{6}\right) \delta=2.65(\mathrm{t}, J=7.1 \mathrm{~Hz}, 2 \mathrm{H}), 3.55(\mathrm{td}, J=7.1 \mathrm{~Hz}, 5.2 \mathrm{~Hz}$, $2 \mathrm{H}), 4.21-4.31(\mathrm{~m}, 2 \mathrm{H}), 4.58(\mathrm{t}, J=5.2 \mathrm{~Hz}, 1 \mathrm{H}), 4.82-4.89(\mathrm{~m}, 2 \mathrm{H}), 6.82-6.90(\mathrm{~m}, 2 \mathrm{H})$, 7.08-7.18 (m, 2H); ${ }^{13} \mathrm{C}$ NMR (101 MHz, DMSO-d $\left.d_{6}\right) \delta=38.1\left(\mathrm{CH}_{2}\right), 62.4\left(\mathrm{CH}_{2} \mathrm{OH}\right), 64.0$ $\left(\mathrm{CH}_{2}\right), 72.1\left(\mathrm{CH}_{2}\right), 114.2\left(2 \mathrm{CH}_{\mathrm{Ar}}\right), 129.9\left(2 \mathrm{CH}_{\mathrm{Ar}}\right), 132.1\left(\mathrm{C}_{\mathrm{q}}\right), 156.1\left(\mathrm{C}_{\mathrm{q}}\right)$; HRMS (ESIMS) $\mathrm{m} / \mathrm{z}$ calcd for $\mathrm{C}_{10} \mathrm{H}_{13} \mathrm{NNaO}_{5}[\mathrm{M}+\mathrm{Na}]^{+}:$250.0691, found: 250.0697; Rf (petroleum ether $/ \mathrm{EtOAc}=6 / 4) 0.63$.

\subsubsection{General Procedure for the Synthesis of the (halidealkyl)phenoxy Nitrates (5a-p)}

To a stirred solution of the appropriate (hydroxyalkyl)phenoxy nitrates $4 \mathbf{a}-\mathbf{o}$ (1.0 Eq.) in dry $\mathrm{CH}_{2} \mathrm{Cl}_{2}$ (35 mL for $7 \mathrm{mmol}$ scale), $10 \mathrm{~mL}$ solution of thionyl chloride (1.2 Eq.) and benzotriazole (BTA, $1.2 \mathrm{Eq}$.) in dry $\mathrm{CH}_{2} \mathrm{Cl}_{2}$ was slowly added, into small portions, according to the methods reported in the literature [70,71], which were adapted to our synthesis in terms of the ratio of reagents, solvent, time of reaction, purification method. Before the reaction was complete, benzotriazole hydrochloride started separating out as a solid. Reaction mixture was stirred further for 20-30 min and after that the solid was filtered off. The filtrate was successively washed with distilled water $(2 \times 50 \mathrm{~mL})$ and brine $(50 \mathrm{~mL})$. To obtain 2-(4-(2-iodooethyl)phenoxy)ethyl nitrate $(5 \mathrm{p})$, to a solution of (hydroxyethyl)phenoxy)ethyl nitrate (4p) (1.0 Eq.) in $\mathrm{CH}_{2} \mathrm{Cl}_{2}(30 \mathrm{~mL})$, imidazole (1.3 Eq.), triphenylphosphine ( $\mathrm{PPh}_{3}, 1.3 \mathrm{Eq}$.) and iodine $(1.3 \mathrm{Eq}$.$) were sequentially added at 0{ }^{\circ} \mathrm{C}$, according to the method descried in the literature [118-120]. The resulting mixture was stirred at room temperature for $6 \mathrm{~h}$ and reaction was quenched by addition of $10 \mathrm{~mL}$ of saturated aqueous $\mathrm{Na}_{2} \mathrm{~S}_{2} \mathrm{O}_{3}$ solution. The combined organic layers were dried over magnesium sulfate, filtered and concentrated under reduced pressure to obtain a crude product that was purified by flash chromatography (silicagel, petroleum ether/EtOAc) to produce the pure products $5 \mathbf{a}-\mathbf{p}$.

2-(4-(Chloromethyl)phenoxy)ethyl nitrate (5a): yellow oil, yield $99 \% ;{ }^{1} \mathrm{H}$ NMR $(400 \mathrm{MHz}$, DMSO- $\left.d_{6}\right) \delta=4.28-4.36(\mathrm{~m}, 2 \mathrm{H}), 4.73(\mathrm{~s}, 2 \mathrm{H}), 4.85-4.93(\mathrm{~m}, 2 \mathrm{H}), 6.97(\mathrm{~d}, J=8.2 \mathrm{~Hz}, 2 \mathrm{H}), 7.38(\mathrm{~d}$, $J=8.2 \mathrm{~Hz}, 2 \mathrm{H}) ;{ }^{13} \mathrm{C}$ NMR $\left(101 \mathrm{MHz}, \mathrm{DMSO}-d_{6}\right) \delta=46.1\left(\mathrm{CH}_{2}\right), 64.0\left(\mathrm{CH}_{2}\right), 72.0\left(\mathrm{CH}_{2}\right), 114.6$ $\left(2 \mathrm{CH}_{\mathrm{Ar}}\right), 130.3\left(\mathrm{C}_{\mathrm{q}}\right), 130.4\left(2 \mathrm{CH}_{\mathrm{Ar}}\right), 157.8\left(\mathrm{C}_{\mathrm{q}}\right)$; HRMS (ESI-MS) $\mathrm{m} / \mathrm{z}$ calcd for $\mathrm{C}_{9} \mathrm{H}_{10} \mathrm{ClNNaO}_{4}$ $[\mathrm{M}+\mathrm{Na}]^{+}:$254.0196, found: 254.0185; Rf (petroleum ether $/ \mathrm{EtOAc}=7 / 3$ ) 0.69 . 
2-(4-(Chloromethyl)-2-fluorophenoxy)ethyl nitrate (5b): pale yellow oil, yield $97 \% ;{ }^{1} \mathrm{H}$ NMR (400 MHz, DMSO- $\left.d_{6}\right) \delta=4.36-4.43(\mathrm{~m}, 2 \mathrm{H}), 4.72(\mathrm{~s}, 2 \mathrm{H}), 4.86-4.93(\mathrm{~m}, 2 \mathrm{H}), 7.16-7.26$ $(\mathrm{m}, 2 \mathrm{H}), 7.33(\mathrm{dd}, J=12.3 \mathrm{~Hz}, 1.7 \mathrm{~Hz}, 1 \mathrm{H}) ;{ }^{13} \mathrm{C}$ NMR $\left(101 \mathrm{MHz}, \mathrm{DMSO}-d_{6}\right) \delta=45.3\left(\mathrm{CH}_{2}\right)$, $65.3\left(\mathrm{CH}_{2}\right), 71.7\left(\mathrm{CH}_{2}\right), 115.2\left(\mathrm{~d}, J=1.9 \mathrm{~Hz}, \mathrm{CH}_{\mathrm{Ar}}\right), 116.7\left(\mathrm{~d}, J=18.7 \mathrm{~Hz}, \mathrm{CH}_{\mathrm{Ar}}\right), 125.5(\mathrm{~d}$, $\left.J=3.5 \mathrm{~Hz}, \mathrm{CH}_{\mathrm{Ar}}\right), 131.3\left(\mathrm{~d}, J=6.6 \mathrm{~Hz}, \mathrm{C}_{\mathrm{q}}\right), 145.7\left(\mathrm{~d}, J=10.6 \mathrm{~Hz}, \mathrm{C}_{\mathrm{q}}\right), 151.19(\mathrm{~d}, J=244.7 \mathrm{~Hz}$, $\left.\mathrm{C}_{\mathrm{q}} \mathrm{F}\right) ;{ }^{19} \mathrm{~F}$ NMR $\left(376 \mathrm{MHz}\right.$, DMSO- $\left.d_{6}\right) \delta=-134.2(\mathrm{dd}, J=12.3 \mathrm{~Hz}, 7.5 \mathrm{~Hz}) ;$ HRMS (ESIMS) $\mathrm{m} / \mathrm{z}$ calcd for $\mathrm{C}_{9} \mathrm{H}_{9} \mathrm{ClFNNaO}_{4}[\mathrm{M}+\mathrm{Na}]^{+}: 272.0102$, found: 272.0096; Rf (petroleum ether $/ \mathrm{EtOAc}=7 / 3) 0.69$.

2-(2-Chloro-4-(chloromethyl)phenoxy)ethyl nitrate (5c): white solid, yield $95 \%$, m.p. $52-54{ }^{\circ} \mathrm{C}$; ${ }^{1} \mathrm{H}$ NMR $\left(400 \mathrm{MHz}\right.$, DMSO- $\left.d_{6}\right) \delta=4.38-4.46(\mathrm{~m}, 2 \mathrm{H}), 4.73(\mathrm{~s}, 2 \mathrm{H}), 4.89-4.95(\mathrm{~m}, 2 \mathrm{H}), 7.19(\mathrm{~d}$, $J=8.5 \mathrm{~Hz}, 1 \mathrm{H}), 7.39(\mathrm{dd}, J=8.5 \mathrm{~Hz}, 2.2 \mathrm{~Hz}, 1 \mathrm{H}), 7.54(\mathrm{~d}, J=2.1 \mathrm{~Hz}, 1 \mathrm{H}) ;{ }^{13} \mathrm{C} \mathrm{NMR}(101 \mathrm{MHz}$, DMSO- $\left.d_{6}\right) \delta=45.1\left(\mathrm{CH}_{2}\right), 65.4\left(\mathrm{CH}_{2}\right), 71.6\left(\mathrm{CH}_{2}\right), 114.1\left(\mathrm{CH}_{\mathrm{Ar}}\right), 121.3\left(\mathrm{C}_{\mathrm{q}}\right), 129.0\left(\mathrm{CH}_{\mathrm{Ar}}\right)$, $130.5\left(\mathrm{CH}_{\mathrm{Ar}}\right), 131.7\left(\mathrm{C}_{\mathrm{q}}\right), 153.1\left(\mathrm{C}_{\mathrm{q}}\right)$; HRMS (ESI-MS) $\mathrm{m} / \mathrm{z}$ calcd for $\mathrm{C}_{9} \mathrm{H}_{9} \mathrm{Cl}_{2} \mathrm{NNaO}_{4}[\mathrm{M}+\mathrm{Na}]^{+}$: 287.9806, found: 287.9798; $\mathrm{Rf}$ (petroleum ether $/ \mathrm{EtOAc}=7 / 3$ ) 0.65.

2-(2,6-Dichloro-4-(chloromethyl)phenoxy)ethyl nitrate (5d): white solid, yield $85 \%$, m.p. $49-51{ }^{\circ} \mathrm{C} ;{ }^{1} \mathrm{H}$ NMR $\left(400 \mathrm{MHz}, \mathrm{DMSO}-d_{6}\right) \delta=4.27-4.38(\mathrm{~m}, 2 \mathrm{H}), 4.74(\mathrm{~s}, 2 \mathrm{H})$, 4.86-4.93 (m, 2H), $7.62(\mathrm{~s}, 2 \mathrm{H}) ;{ }^{13} \mathrm{C}$ NMR $\left(101 \mathrm{MHz}, \mathrm{DMSO}-d_{6}\right) \delta=43.9\left(\mathrm{CH}_{2}\right), 69.5$ $\left(\mathrm{CH}_{2}\right), 72.4\left(\mathrm{CH}_{2}\right), 128.2\left(2 \mathrm{C}_{\mathrm{q}}\right), 129.7\left(2 \mathrm{CH}_{\mathrm{Ar}}\right), 136.3\left(\mathrm{C}_{\mathrm{q}}\right), 149.9\left(\mathrm{C}_{\mathrm{q}}\right)$; HRMS (ESI-MS) $\mathrm{m} / \mathrm{z}$ calcd for $\mathrm{C}_{9} \mathrm{H}_{8} \mathrm{Cl}_{3} \mathrm{NNaO}_{4}[\mathrm{M}+\mathrm{Na}]^{+}$: 321.9417, found: 321.9406; Rf (petroleum ether $/ \mathrm{EtOAc}=8 / 2) 0.70$.

2-(4-(Chloromethyl)-2-nitrophenoxy)ethyl nitrate (5e): greenish yellow solid, yield $90 \%$, m.p. $56-58{ }^{\circ} \mathrm{C} ;{ }^{1} \mathrm{H}$ NMR $\left(400 \mathrm{MHz}, \mathrm{DMSO}-d_{6}\right) \delta=4.49-4.55(\mathrm{~m}, 2 \mathrm{H}), 4.81(\mathrm{~s}, 2 \mathrm{H}), 4.86-4.94$ $(\mathrm{m}, 2 \mathrm{H}), 7.42(\mathrm{~d}, J=8.7 \mathrm{~Hz}, 1 \mathrm{H}), 7.74(\mathrm{dd}, J=8.7 \mathrm{~Hz}, 2.3 \mathrm{~Hz}, 1 \mathrm{H}), 8.00(\mathrm{~d}, J=2.3 \mathrm{~Hz}, 1 \mathrm{H})$; ${ }^{13} \mathrm{C}$ NMR $\left(101 \mathrm{MHz}, \mathrm{DMSO}-d_{6}\right) \delta=44.4\left(\mathrm{CH}_{2}\right), 66.1\left(\mathrm{CH}_{2}\right), 71.3\left(\mathrm{CH}_{2}\right), 115.8\left(\mathrm{CH}_{\mathrm{Ar}}\right), 125.3$ $\left(\mathrm{CH}_{\mathrm{Ar}}\right), 131.0\left(\mathrm{C}_{\mathrm{q}}\right), 133.0\left(\mathrm{CH}_{\mathrm{Ar}}\right), 139.2\left(\mathrm{C}_{\mathrm{q}}\right), 150.4\left(\mathrm{C}_{\mathrm{q}}\right)$; HRMS (ESI-MS) $\mathrm{m} / \mathrm{z}$ calcd for $\mathrm{C}_{9} \mathrm{H}_{9} \mathrm{ClN}_{2} \mathrm{NaO}_{6}[\mathrm{M}+\mathrm{Na}]^{+}: 299.0047$, found: 299.0044; $\mathrm{Rf}\left(\mathrm{CH}_{2} \mathrm{Cl}_{2}\right)$ 0.70.

2-(4-(Chloromethyl)-2-methoxyphenoxy)ethyl nitrate (5f): white solid, yield 85\%, m.p. $54-56{ }^{\circ} \mathrm{C} ;{ }^{1} \mathrm{H}$ NMR $\left(250 \mathrm{MHz}, \mathrm{DMSO}-d_{6}\right) \delta=3.77(\mathrm{~s}, 3 \mathrm{H}), 4.20-4.35(\mathrm{~m}, 2 \mathrm{H}), 4.71(\mathrm{~s}, 2 \mathrm{H})$, 4.80-4.91 (m, 2H), 6.96-6.99 (m, 2H), $7.07(\mathrm{~s}, 1 \mathrm{H}) ;{ }^{13} \mathrm{C}$ NMR $\left(101 \mathrm{MHz}\right.$, DMSO- $\left.d_{6}\right) \delta=46.5$ $\left(\mathrm{CH}_{2}\right), 55.6\left(\mathrm{CH}_{3}\right), 65.0\left(\mathrm{CH}_{2}\right), 72.0\left(\mathrm{CH}_{2}\right), 112.9\left(\mathrm{CH}_{\mathrm{Ar}}\right), 113.7\left(\mathrm{CH}_{\mathrm{Ar}}\right), 121.4\left(\mathrm{CH}_{\mathrm{Ar}}\right), 131.0$ $\left(\mathrm{C}_{\mathrm{q}}\right), 147.3\left(\mathrm{C}_{\mathrm{q}}\right), 149.0\left(\mathrm{C}_{\mathrm{q}}\right)$. HRMS (ESI-MS) $\mathrm{m} / \mathrm{z}$ calcd for $\mathrm{C}_{10} \mathrm{H}_{12} \mathrm{ClNNaO}_{5}[\mathrm{M}+\mathrm{Na}]^{+}$: 284.0302, found: 284.0299; Rf (petroleum ether/EtOAc = 5/5) 0.76 .

2-(4-(Chloromethyl)-2-ethoxyphenoxy)ethyl nitrate (5g): white solid, yield 84\%, m.p. 57-60 ${ }^{\circ} \mathrm{C} ;{ }^{1} \mathrm{H}$ NMR $\left(250 \mathrm{MHz}\right.$, DMSO- $\left.d_{6}\right) \delta=1.32(\mathrm{t}, J=6.9 \mathrm{~Hz}, 3 \mathrm{H}), 4.02(\mathrm{q}, J=6.9 \mathrm{~Hz}$, $2 \mathrm{H}), 4.24-4.33(\mathrm{~m}, 2 \mathrm{H}), 4.70(\mathrm{~s}, 2 \mathrm{H}), 4.81-4.92(\mathrm{~m}, 2 \mathrm{H}), 6.95-6.99(\mathrm{~m}, 2 \mathrm{H}), 7.06(\mathrm{~d}, J=1.7 \mathrm{~Hz}$, $0.6 \mathrm{~Hz}, 1 \mathrm{H}) ;{ }^{13} \mathrm{C}$ NMR $\left(101 \mathrm{MHz}, \mathrm{DMSO}-d_{6}\right) \delta=14.6\left(\mathrm{CH}_{3}\right), 46.4\left(\mathrm{CH}_{2}\right), 63.9\left(\mathrm{CH}_{2}\right), 65.4$ $\left(\mathrm{CH}_{2}\right), 72.0\left(\mathrm{CH}_{2}\right), 114.4\left(\mathrm{CH}_{\mathrm{Ar}}\right), 114.5\left(\mathrm{CH}_{\mathrm{Ar}}\right), 121.5\left(\mathrm{CH}_{\mathrm{Ar}}\right), 131.2\left(\mathrm{C}_{\mathrm{q}}\right), 147.6\left(\mathrm{C}_{\mathrm{q}}\right), 148.4$ $\left(\mathrm{C}_{\mathrm{q}}\right.$ ); HRMS (ESI-MS) $\mathrm{m} / \mathrm{z}$ calcd for $\mathrm{C}_{11} \mathrm{H}_{14} \mathrm{ClNNaO}_{5}$ [M+Na] ${ }^{+}:$298.0458, found: 298.0456; $\mathrm{Rf}$ (petroleum ether $/ \mathrm{EtOAc}=7 / 3$ ) 0.72 .

2-(4-(Chloromethyl)-2,6-dimethoxyphenoxy)ethyl nitrate (5h): white solid, yield 86\%, m.p. 59-61 ${ }^{\circ} \mathrm{C} ;{ }^{1} \mathrm{H}$ NMR $\left(400 \mathrm{MHz}, \mathrm{DMSO}-d_{6}\right) \delta=3.76(\mathrm{~s}, 6 \mathrm{H}), 4.09-4.17(\mathrm{~m}, 2 \mathrm{H}), 4.70(\mathrm{~s}$, 2H), 4.72-4.78 (m, 2H), $6.78(\mathrm{~s}, 2 \mathrm{H}) ;{ }^{13} \mathrm{C}$ NMR $\left(101 \mathrm{MHz}, \mathrm{DMSO}-d_{6}\right) \delta=46.6\left(\mathrm{CH}_{2}\right), 55.9$ $\left(2 \mathrm{CH}_{3}\right), 68.5\left(\mathrm{CH}_{2}\right), 72.9\left(\mathrm{CH}_{2}\right), 106.1\left(2 \mathrm{CH}_{\mathrm{Ar}}\right), 133.6\left(\mathrm{C}_{\mathrm{q}}\right), 135.6\left(\mathrm{C}_{\mathrm{q}}\right), 152.7\left(2 \mathrm{C}_{\mathrm{q}}\right)$; HRMS (ESI-MS) $\mathrm{m} / \mathrm{z}$ calcd for $\mathrm{C}_{11} \mathrm{H}_{15} \mathrm{ClNO}_{6}[\mathrm{M}+\mathrm{H}]^{+}: 292.0588$, found: 292.0580; $\mathrm{Rf}$ (petroleum ether $/ \mathrm{EtOAc}=7 / 3) 0.61$.

2-(3-(Chloromethyl)phenoxy)ethyl nitrate (5i): pale yellow oil, yield $88 \% ;{ }^{1} \mathrm{H}$ NMR $\left(250 \mathrm{MHz}, \mathrm{DMSO}-d_{6}\right) \delta=4.26-4.37(\mathrm{~m}, 2 \mathrm{H}), 4.72(\mathrm{~s}, 2 \mathrm{H}), 4.83-4.95(\mathrm{~m}, 2 \mathrm{H}), 6.94(\mathrm{ddd}$, $J=8.2 \mathrm{~Hz}, 2.6 \mathrm{~Hz}, 1.0 \mathrm{~Hz}, 1 \mathrm{H}), 7.00-7.09(\mathrm{~m}, 2 \mathrm{H}), 7.24-7.38(\mathrm{~m}, 1 \mathrm{H}) ;{ }^{13} \mathrm{C} \mathrm{NMR}(101 \mathrm{MHz}$, DMSO- $\left.d_{6}\right) \delta=45.9\left(\mathrm{CH}_{2}\right), 64.0\left(\mathrm{CH}_{2}\right), 71.9\left(\mathrm{CH}_{2}\right), 114.4\left(\mathrm{CH}_{\mathrm{Ar}}\right), 114.9\left(\mathrm{CH}_{\mathrm{Ar}}\right), 121.6\left(\mathrm{CH}_{\mathrm{Ar}}\right)$, $129.8\left(\mathrm{CH}_{\mathrm{Ar}}\right), 139.2\left(\mathrm{C}_{\mathrm{q}}\right), 157.9\left(\mathrm{C}_{\mathrm{q}}\right)$; HRMS (ESI-MS) $\mathrm{m} / \mathrm{z}$ calcd for $\mathrm{C}_{9} \mathrm{H}_{11} \mathrm{ClNO}_{4}[\mathrm{M}+\mathrm{H}]^{+}$: 232.0377, found: 232.0371; Rf (petroleum ether $/$ EtOAc $=8 / 2$ ) 0.60 .

2-(5-(Chloromethyl)-2-methoxyphenoxy)ethyl nitrate (5j): yellow oil, yield $92 \%,{ }^{1} \mathrm{H}$ NMR $\left(250 \mathrm{MHz}, \mathrm{DMSO}-d_{6}\right) \delta=3.77(\mathrm{~s}, 3 \mathrm{H}), 4.22-4.33(\mathrm{~m}, 2 \mathrm{H}), 4.70(\mathrm{~s}, 2 \mathrm{H}), 4.79-4.92(\mathrm{~m}$, $2 \mathrm{H}), 6.97(\mathrm{~d}, J=8.2 \mathrm{~Hz}, 1 \mathrm{H}), 7.02(\mathrm{~d}, J=1.9 \mathrm{~Hz}, 1 \mathrm{H}), 7.09(\mathrm{~d}, J=1.9 \mathrm{~Hz}, 1 \mathrm{H}) ;{ }^{13} \mathrm{C}$ 
NMR (101 MHz, DMSO-d 6$) \delta=46.5\left(\mathrm{CH}_{2}\right), 55.6\left(\mathrm{CH}_{3}\right), 65.1\left(\mathrm{CH}_{2}\right), 72.0\left(\mathrm{CH}_{2}\right), 112.1$ $\left(\mathrm{CH}_{\mathrm{Ar}}\right), 114.7\left(\mathrm{CH}_{\mathrm{Ar}}\right), 122.5\left(\mathrm{CH}_{\mathrm{Ar}}\right), 129.9\left(\mathrm{C}_{\mathrm{q}}\right), 147.1\left(\mathrm{C}_{\mathrm{q}}\right), 149.2\left(\mathrm{C}_{\mathrm{q}}\right)$; HRMS (ESI-MS) $\mathrm{m} / \mathrm{z}$ calcd for $\mathrm{C}_{10} \mathrm{H}_{12} \mathrm{ClNNaO}_{5}[\mathrm{M}+\mathrm{Na}]^{+}$: 284.0302, found: 284.0297; Rf (petroleum ether $/ \mathrm{EtOAc}=8 / 2) 0.45$.

2-(5-(Chloromethyl)-2-nitrophenoxy)ethyl nitrate (5k): brown solid, yield 91\%, m.p. $51-53{ }^{\circ} \mathrm{C} ;{ }^{1} \mathrm{H}$ NMR $\left(400 \mathrm{MHz}, \mathrm{DMSO}-d_{6}\right) \delta=4.47-4.56(\mathrm{~m}, 2 \mathrm{H}), 4.81(\mathrm{~s}, 2 \mathrm{H}), 4.88-4.97$ $(\mathrm{m}, 2 \mathrm{H}), 7.23(\mathrm{dd}, J=8.3 \mathrm{~Hz}, 1.6 \mathrm{~Hz}, 1 \mathrm{H}), 7.50(\mathrm{~d}, J=1.6 \mathrm{~Hz}, 1 \mathrm{H}), 7.91(\mathrm{~d}, J=8.3 \mathrm{~Hz}$, $1 \mathrm{H}) ;{ }^{13} \mathrm{C}$ NMR $\left(101 \mathrm{MHz}, \mathrm{DMSO}-d_{6}\right) \delta=44.7\left(\mathrm{CH}_{2}\right), 66.1\left(\mathrm{CH}_{2}\right), 71.3\left(\mathrm{CH}_{2}\right), 115.6$ $\left(\mathrm{CH}_{\mathrm{Ar}}\right), 121.5\left(\mathrm{CH}_{\mathrm{Ar}}\right), 125.5\left(\mathrm{CH}_{\mathrm{Ar}}\right), 139.0\left(\mathrm{C}_{\mathrm{q}}\right), 144.3\left(\mathrm{C}_{\mathrm{q}}\right), 150.5\left(\mathrm{C}_{\mathrm{q}}\right)$; HRMS (ESI-MS) $\mathrm{m} / \mathrm{z}$ calcd for $\mathrm{C}_{9} \mathrm{H}_{9} \mathrm{ClN}_{2} \mathrm{NaO}_{6}[\mathrm{M}+\mathrm{Na}]^{+}$: 299.0047, found: 299.0043; Rf (petroleum ether $\left./ \mathrm{CH}_{2} \mathrm{Cl}_{2}=3 / 7\right)$ 0.67. 2-(2-Chloro-3-(chloromethyl)-6-methoxyphenoxy)ethyl nitrate (5l): pale yellow oil, yield $96 \% ;{ }^{1} \mathrm{H}$ NMR $\left(400 \mathrm{MHz}, \mathrm{DMSO}-\mathrm{d}_{6}\right) \delta=3.83(\mathrm{~s}, 3 \mathrm{H}), 4.21-4.30$ $(\mathrm{m}, 2 \mathrm{H}), 4.79(\mathrm{~s}, 2 \mathrm{H}), 4.80-4.87(\mathrm{~m}, 2 \mathrm{H}), 7.08(\mathrm{~d}, J=8.6 \mathrm{~Hz}, 1 \mathrm{H}), 7.36(\mathrm{~d}, J=8.6 \mathrm{~Hz}$, $1 \mathrm{H}) ; .{ }^{13} \mathrm{C}$ NMR $\left(101 \mathrm{MHz}, \mathrm{DMSO}-d_{6}\right) \delta=44.2\left(\mathrm{CH}_{2}\right), 56.2\left(\mathrm{CH}_{3}\right), 68.8\left(\mathrm{CH}_{2}\right), 72.7\left(\mathrm{CH}_{2}\right)$, 111.4 $\left(\mathrm{CH}_{\mathrm{Ar}}\right), 126.9\left(\mathrm{CH}_{\mathrm{Ar}}\right), 127.6\left(\mathrm{C}_{\mathrm{q}}\right), 127.7\left(\mathrm{C}_{\mathrm{q}}\right), 143.4\left(\mathrm{C}_{\mathrm{q}}\right), 153.5\left(\mathrm{C}_{\mathrm{q}}\right)$; HRMS (ESIMS) $\mathrm{m} / \mathrm{z}$ calcd for $\mathrm{C}_{10} \mathrm{H}_{11} \mathrm{Cl}_{2} \mathrm{NNaO}_{5}[\mathrm{M}+\mathrm{Na}]^{+}$: 317.9912, found: 317.9906; Rf (petroleum ether $/ \mathrm{EtOAc}=7 / 3) 0.67$.

2-(2-Bromo-3-(chloromethyl)-6-methoxyphenoxy)ethyl nitrate (5m): greenish yellow oil, yield $95 \% ;{ }^{1} \mathrm{H}$ NMR $\left(400 \mathrm{MHz}, \mathrm{DMSO}-d_{6}\right) \delta=3.83(\mathrm{~s}, 3 \mathrm{H}), 4.19-4.30(\mathrm{~m}, 2 \mathrm{H}), 4.80(\mathrm{~s}, 2 \mathrm{H})$, 4.81-4.88 (m, 2H), $7.12(\mathrm{~d}, J=8.5 \mathrm{~Hz}, 1 \mathrm{H}), 7.38(\mathrm{~d}, J=8.5 \mathrm{~Hz}, 1 \mathrm{H}) ;{ }^{13} \mathrm{C} \mathrm{NMR}(101 \mathrm{MHz}$, DMSO-d $\left.d_{6}\right) \delta=46.8\left(\mathrm{CH}_{2}\right), 56.2\left(\mathrm{CH}_{3}\right), 68.7\left(\mathrm{CH}_{2}\right), 72.7\left(\mathrm{CH}_{2}\right), 112.1\left(\mathrm{CH}_{\mathrm{Ar}}\right), 119.3\left(\mathrm{C}_{\mathrm{q}}\right), 127.3$ $\left(\mathrm{CH}_{\mathrm{Ar}}\right), 129.3\left(\mathrm{C}_{\mathrm{q}}\right), 144.5\left(\mathrm{C}_{\mathrm{q}}\right), 153.3\left(\mathrm{C}_{\mathrm{q}}\right)$; HRMS (ESI-MS) $\mathrm{m} / \mathrm{z}$ calcd for $\mathrm{C}_{10} \mathrm{H}_{11} \mathrm{BrClNNaO}_{5}$ $[\mathrm{M}+\mathrm{Na}]^{+}:$361.9407, found: 361.9401; Rf (petroleum ether $/$EtOAc $=6 / 4$ ) 0.71 .

2-(2-(Chloromethyl)phenoxy)ethyl nitrate (5n): yellow oil, yield $83 \% ;{ }^{1} \mathrm{H}$ NMR $(250 \mathrm{MHz}$, DMSO- $\left.d_{6}\right) \delta=4.34-4.42(\mathrm{~m}, 2 \mathrm{H}), 4.69(\mathrm{~s}, 2 \mathrm{H}), 4.89-4.97(\mathrm{~m}, 2 \mathrm{H}), 6.92-7.11(\mathrm{~m}, 2 \mathrm{H}), 7.29-7.46$ $(\mathrm{m}, 2 \mathrm{H}) ;{ }^{13} \mathrm{C}$ NMR $\left(101 \mathrm{MHz}, \mathrm{DMSO}-d_{6}\right) \delta=40.5\left(\mathrm{CH}_{2}\right), 64.8\left(\mathrm{CH}_{2}\right), 72.0\left(\mathrm{CH}_{2}\right), 111.4$ $\left(\mathrm{CH}_{\mathrm{Ar}}\right), 114.8\left(\mathrm{CH}_{\mathrm{Ar}}\right), 127.5\left(\mathrm{CH}_{\mathrm{Ar}}\right), 129.5\left(\mathrm{CH}_{\mathrm{Ar}}\right), 132.7\left(\mathrm{C}_{\mathrm{q}}\right), 154.5\left(\mathrm{C}_{\mathrm{q}}\right)$; HRMS (ESIMS) $\mathrm{m} / \mathrm{z}$ calcd for $\mathrm{C}_{9} \mathrm{H}_{10} \mathrm{ClNNaO}_{4}[\mathrm{M}+\mathrm{Na}]^{+}: 254.0196$, found: 254.0193; $\mathrm{Rf}$ (petroleum ether $/ \mathrm{EtOAc}=8 / 2) 0.64$.

2-(4-Bromo-2-(bromomethyl)phenoxy)ethyl nitrate (5o): pale yellow oil, yield $89 \% ;{ }^{1} \mathrm{H}$ NMR (400 MHz, DMSO- $\left.d_{6}\right) \delta=4.33-4.41(\mathrm{~m}, 2 \mathrm{H}), 4.67(\mathrm{~s}, 2 \mathrm{H}), 4.87-4.96(\mathrm{~m}, 2 \mathrm{H}), 7.07(\mathrm{~d}$, $J=8.7 \mathrm{~Hz}, 1 \mathrm{H}), 7.52(\mathrm{dd}, J=8.8 \mathrm{~Hz}, 2.5 \mathrm{~Hz}, 1 \mathrm{H}), 7.62(\mathrm{~d}, J=2.6 \mathrm{~Hz}, 1 \mathrm{H}) ;{ }^{13} \mathrm{C} \mathrm{NMR}(101 \mathrm{MHz}$, DMSO- $\left.d_{6}\right) \delta=40.4\left(\mathrm{CH}_{2}\right), 65.2\left(\mathrm{CH}_{2}\right), 71.7\left(\mathrm{CH}_{2}\right), 112.3\left(\mathrm{C}_{\mathrm{q}}\right), 114.9\left(\mathrm{CH}_{\mathrm{Ar}}\right), 128.3\left(\mathrm{C}_{\mathrm{q}}\right)$, 132.7 $\left(\mathrm{CH}_{\mathrm{Ar}}\right), 133.1\left(\mathrm{CH}_{\mathrm{Ar}}\right), 155.2\left(\mathrm{C}_{\mathrm{q}}\right)$; HRMS (ESI-MS) m/z calcd for $\mathrm{C}_{9} \mathrm{H}_{9} \mathrm{BrClNNaO}_{4}$ $[\mathrm{M}+\mathrm{Na}]^{+}:$331.9301, found: 331.9310; Rf (petroleum ether $\left./ \mathrm{EtOAc}=8 / 2\right) 0.55$.

2-(4-(2-Iodooethyl)phenoxy)ethyl nitrate (5p): white solid, yield 79\%, m.p. $57-59{ }^{\circ} \mathrm{C} ;{ }^{1} \mathrm{H}$ $\operatorname{NMR}\left(400 \mathrm{MHz}, \mathrm{DMSO}-d_{6}\right) \delta=3.05(\mathrm{t}, J=7.4 \mathrm{~Hz}, 2 \mathrm{H}), 3.42(\mathrm{t}, J=7.4 \mathrm{~Hz}, 2 \mathrm{H}), 4.23-4.31(\mathrm{~m}$, $2 \mathrm{H}), 4.81-4.91(\mathrm{~m}, 2 \mathrm{H}), 6.89(\mathrm{~d}, J=7.4 \mathrm{~Hz}, 2 \mathrm{H}), 7.18(\mathrm{~d}, J=7.6 \mathrm{~Hz}, 2 \mathrm{H}) ;{ }^{13} \mathrm{C}$ NMR $(101 \mathrm{MHz}$, DMSO- $\left.d_{6}\right) \delta=8.7\left(\mathrm{CH}_{2}\right), 38.4\left(\mathrm{CH}_{2}\right), 63.9\left(\mathrm{CH}_{2}\right), 72.0\left(\mathrm{CH}_{2}\right), 114.4\left(2 \mathrm{CH}_{\mathrm{Ar}}\right), 129.6\left(2 \mathrm{CH}_{\mathrm{Ar}}\right)$, $133.3\left(\mathrm{C}_{\mathrm{q}}\right), 156.5\left(\mathrm{C}_{\mathrm{q}}\right)$; HRMS (ESI-MS) $\mathrm{m} / \mathrm{z}$ calcd for $\mathrm{C}_{10} \mathrm{H}_{13} \mathrm{INO}_{4}[\mathrm{M}+\mathrm{H}]^{+}: 337.9889$, found: 337.9884; Rf (petroleum ether $/ \mathrm{EtOAc}=8 / 2$ ) 0.60 .

3.1.6. Synthesis of (4-chlorobenzoyl)-5-methoxy-2-methyl-1H-indol-3-yl)methyl)-1,3, 4-oxadiazol (7)

To a suspension of indomethacin hydrazide (6) [99] (1.0 Eq.) in $\mathrm{CH}_{3} \mathrm{CN}(150 \mathrm{~mL}$ for $12 \mathrm{mmol} \mathrm{scale}), \mathrm{Et}_{3} \mathrm{~N}\left(2.0 \mathrm{Eq}\right.$.) and $\mathrm{CS}_{2}(2.0 \mathrm{Eq}$.) were slowly added, into small portions, according to the method reported in the literature [121-124], which was adapted to our synthesis in terms of the ratio of reagents, solvent, time of reaction and purification method. The mixture was stirred for $3 \mathrm{~h}$ under reflux till hydrogen sulfide formation was stopped. After cooling at room temperature, the solvent was removed under reduced pressure and the residue was dissolved in EtOAc $(40 \mathrm{~mL})$ and acidified with aqueous diluted $\mathrm{HCl} 0.5 \mathrm{M}$ solution $(10 \mathrm{~mL})$. The organic layer was separated and was successively washed with distilled water $(3 \times 50 \mathrm{~mL})$ and finally with brine $(50 \mathrm{~mL})$. The combined organic layers were dried over anhydrous magnesium sulfate, filtered and concentrated under reduced pressure. The crude product was purified by flash column chromatography to give the 
pure product: white solid, yield 92\%, m.p. $199-201{ }^{\circ} \mathrm{C} ;{ }^{1} \mathrm{H}$ NMR $\left(400 \mathrm{MHz}, \mathrm{DMSO}-d_{6}\right)$ $\delta=2.29(\mathrm{~s}, 3 \mathrm{H}), 3.76(\mathrm{~s}, 3 \mathrm{H}), 4.25(\mathrm{~s}, 2 \mathrm{H}), 6.74(\mathrm{dd}, J=9.0 \mathrm{~Hz}, 2.5 \mathrm{~Hz}, 1 \mathrm{H}), 6.90(\mathrm{~d}, J=9.0 \mathrm{~Hz}$, $1 \mathrm{H}), 7.12(\mathrm{~d}, J=2.5 \mathrm{~Hz}, 1 \mathrm{H}), 7.56-7.76(\mathrm{~m}, 4 \mathrm{H}), 14.36(\mathrm{~s}, 1 \mathrm{H}) ;{ }^{13} \mathrm{C}$ NMR $\left(101 \mathrm{MHz}, \mathrm{DMSO}-d_{6}\right)$ $\delta=13.1\left(\mathrm{CH}_{3}\right), 20.4\left(\mathrm{CH}_{2}\right), 55.4\left(\mathrm{CH}_{3}\right), 101.6\left(\mathrm{CH}_{\mathrm{Ar}}\right), 111.1\left(\mathrm{C}_{\mathrm{q}}\right), 111.6\left(\mathrm{CH}_{\mathrm{Ar}}\right), 114.7\left(\mathrm{CH}_{\mathrm{Ar}}\right)$, $129.1\left(2 \mathrm{CH}_{\mathrm{Ar}}\right), 129.9\left(\mathrm{C}_{\mathrm{q}}\right), 130.2\left(\mathrm{C}_{\mathrm{q}}\right), 131.2\left(2 \mathrm{CH}_{\mathrm{Ar}}\right), 133.9\left(\mathrm{C}_{\mathrm{q}}\right), 136.1\left(\mathrm{C}_{\mathrm{q}}\right), 137.7\left(\mathrm{C}_{\mathrm{q}}\right), 155.6$ $\left(\mathrm{C}_{\mathrm{q}}\right), 162.3\left(\mathrm{C}_{\mathrm{q}} \mathrm{S}\right), 167.8\left(\mathrm{C}_{\mathrm{q}}\right), 177.8\left(\mathrm{C}_{\mathrm{q}}\right)$; HRMS (ESI-MS) $\mathrm{m} / \mathrm{z}$ calcd for $\mathrm{C}_{20} \mathrm{H}_{17} \mathrm{ClN}_{3} \mathrm{O}_{3} \mathrm{~S}$ $[\mathrm{M}+\mathrm{H}]^{+}:$414.0679, found: 414.0680; $\mathrm{Rf}\left(\mathrm{CH}_{2} \mathrm{Cl}_{2} / \mathrm{CH}_{3} \mathrm{OH}=9.8 / 0.2\right) 0.52$.

3.1.7. General Procedure for the Synthesis of the Nitric Oxide-Releasing Indomethacin Derivatives with 2-mercapto-1,3,4-oxadiazol Scaffold (8a-p)

To a suspension of (4-chlorobenzoyl)-5-methoxy-2-methyl-1H-indol-3-yl)methyl)1,3,4-oxadiazol (7) (1.0 Eq.) in acetonitrile (70 $\mathrm{mL}$ for $4 \mathrm{mmol}$ scale), the corresponding (halidealkyl)phenoxy nitrates 5a-p (1.1 Eq.) were added in one portion followed by $\mathrm{Et}_{2} \mathrm{~N}$ being added drop wise(1.5 Eq.), according to the method reported in the literature, which was adapted to our synthesis in terms of the ratio of reagents, solvent, time of reaction and purification method $[125,126]$. The mixture was stirred at room temperature for 3-6 $\mathrm{h}$ and then the solvent was removed under reduced pressure. The residue was taken up with EtOAc $(50 \mathrm{~mL})$ and successively washed with distilled water $(2 \times 50 \mathrm{~mL})$ and finally with brine $(50 \mathrm{~mL})$. The combined organic layers were dried over magnesium sulfate and solvent was removed under reduced pressure to obtain a crude product that was purified by flash column chromatography to produce the pure products $8 \mathbf{a}-\mathbf{p}$.

2-(4-((5-((1-(4-Chlorobenzoyl)-5-methoxy-2-methyl-1H-indol-3-yl)methyl)-1,3,4oxadiazol-2-yl)thio)methyl)phenoxy)ethyl nitrate (8a): pale yellow solid, yield 88\%, m.p. $108-110{ }^{\circ} \mathrm{C} ;{ }^{1} \mathrm{H}$ NMR $\left(250 \mathrm{MHz}, \mathrm{DMSO}-\mathrm{d}_{6}\right) \delta=2.29(\mathrm{~s}, 3 \mathrm{H}), 3.74(\mathrm{~s}, 3 \mathrm{H}), 4.19-4.28$ $(\mathrm{m}, 2 \mathrm{H}), 4.36(\mathrm{~s}, 2 \mathrm{H}), 4.37(\mathrm{~s}, 2 \mathrm{H}), 4.79-4.91(\mathrm{~m}, 2 \mathrm{H}), 6.74(\mathrm{dd}, \mathrm{J}=9.0 \mathrm{~Hz}, 2.5 \mathrm{~Hz}, 1 \mathrm{H})$, 6.73-6.86 (m, 2H), $6.93(\mathrm{dd}, \mathrm{J}=9.0 \mathrm{~Hz}, 0.5 \mathrm{~Hz}, 1 \mathrm{H}), 7.09(\mathrm{dd}, \mathrm{J}=2.6 \mathrm{~Hz}, 0.5 \mathrm{~Hz}, 1 \mathrm{H})$, 7.14-7.30 (m, 2H), 7.54-7.76 (m, 4H); ${ }^{13} \mathrm{C}$ NMR (101 MHz, DMSO-d 6$) \delta=13.1\left(\mathrm{CH}_{3}\right), 20.0$ $\left(\mathrm{CH}_{2}\right), 35.4\left(\mathrm{CH}_{2}\right), 55.4\left(\mathrm{CH}_{3}\right), 64.0\left(\mathrm{CH}_{2}\right), 71.9\left(\mathrm{CH}_{2}\right), 101.5\left(\mathrm{CH}_{\mathrm{Ar}}\right), 111.6\left(\mathrm{CH}_{\mathrm{Ar}}\right), 111.9$ $\left(\mathrm{C}_{\mathrm{q}}\right), 114.4\left(2 \mathrm{CH}_{\mathrm{Ar}}\right), 114.7\left(\mathrm{CH}_{\mathrm{Ar}}\right), 128.9\left(\mathrm{C}_{\mathrm{q}}\right), 129.1\left(2 \mathrm{CH}_{\mathrm{Ar}}\right), 129.9\left(\mathrm{C}_{\mathrm{q}}\right), 130.3\left(2 \mathrm{CH}_{\mathrm{Ar}}\right)$, $131.2\left(2 \mathrm{CH}_{\mathrm{Ar}}\right), 133.9\left(\mathrm{C}_{\mathrm{q}}\right), 135.7\left(\mathrm{C}_{\mathrm{q}}\right), 137.8\left(\mathrm{C}_{\mathrm{q}}\right), 155.6\left(\mathrm{C}_{\mathrm{q}}\right), 157.3\left(\mathrm{C}_{\mathrm{q}}\right), 163.0\left(\mathrm{C}_{\mathrm{q}}\right), 166.1$ $\left(2 \mathrm{C}_{\mathrm{q}}\right), 167.9\left(\mathrm{C}_{\mathrm{q}}\right)$; HRMS (ESI-MS) $\mathrm{m} / \mathrm{z}$ calcd for $\mathrm{C}_{29} \mathrm{H}_{26} \mathrm{ClN}_{4} \mathrm{O}_{7} \mathrm{~S}[\mathrm{M}+\mathrm{H}]^{+}: 609.1211$, found: $609.1205 ; \mathrm{Rf}$ (petroleum ether $/ \mathrm{EtOAc}=4 / 6) 0.66$.

2-(4-(((5-((1-(4-Chlorobenzoyl)-5-methoxy-2-methyl-1H-indol-3-yl)methyl)-1,3,4oxadiazol-2-yl)thio)methyl)-2-fluorophenoxy)ethyl nitrate (8b): pale yellow solid, yield 86\%, m.p. $105-107^{\circ} \mathrm{C} ;{ }^{1} \mathrm{H}$ NMR (400 MHz, DMSO-d 6 ) $\delta=2.28$ (s, 3H), $3.74(\mathrm{~s}, 3 \mathrm{H}), 4.29$ $4.34(\mathrm{~m}, 2 \mathrm{H}), 4.36(\mathrm{~s}, 2 \mathrm{H}), 4.38(\mathrm{~s}, 2 \mathrm{H}), 4.81-4.93(\mathrm{~m}, 2 \mathrm{H}), 6.74(\mathrm{dd}, \mathrm{J}=9.0 \mathrm{~Hz}, 2.5 \mathrm{~Hz}, 1 \mathrm{H})$, $6.92(\mathrm{~d}, \mathrm{~J}=9.0 \mathrm{~Hz}, 1 \mathrm{H}), 7.01(\mathrm{t}, \mathrm{J}=8.5 \mathrm{~Hz}, 1 \mathrm{H}), 7.07(\mathrm{dd}, \mathrm{J}=9.0 \mathrm{~Hz}, 2.0 \mathrm{~Hz}, 2 \mathrm{H}), 7.25(\mathrm{dd}$, $\mathrm{J}=12.2 \mathrm{~Hz}, 2.0 \mathrm{~Hz}, 1 \mathrm{H}), 7.59-7.73(\mathrm{~m}, 4 \mathrm{H}) ;{ }^{13} \mathrm{C} \mathrm{NMR}\left(101 \mathrm{MHz}, \mathrm{DMSO}-\mathrm{d}_{6}\right) \delta=13.0\left(\mathrm{CH}_{3}\right)$, $20.0\left(\mathrm{CH}_{2}\right), 34.9\left(\mathrm{CH}_{2}\right), 55.4\left(\mathrm{CH}_{3}\right), 65.2\left(\mathrm{CH}_{2}\right), 71.7\left(\mathrm{CH}_{2}\right), 101.5\left(\mathrm{CH}_{\mathrm{Ar}}\right), 111.6\left(\mathrm{CH}_{\mathrm{Ar}}\right)$, $111.8\left(\mathrm{C}_{\mathrm{q}}\right), 114.7\left(\mathrm{CH}_{\mathrm{Ar}}\right), 114.9\left(\mathrm{~d}, \mathrm{~J}=1.8 \mathrm{~Hz}, \mathrm{CH}_{\mathrm{Ar}}\right), 116.6\left(\mathrm{CH}_{\mathrm{Ar}}\right), 116.8\left(\mathrm{C}_{\mathrm{q}}\right), 125.3(\mathrm{~d}$, $\left.\mathrm{J}=3.4 \mathrm{~Hz}, \mathrm{CH}_{\mathrm{Ar}}\right), 129.0\left(2 \mathrm{CH}_{\mathrm{Ar}}\right), 129.9\left(\mathrm{C}_{\mathrm{q}}\right), 130.2\left(\mathrm{~d}, \mathrm{~J}=3.3 \mathrm{~Hz}, \mathrm{C}_{\mathrm{q}}\right), 131.2\left(2 \mathrm{CH}_{\mathrm{Ar}}\right), 133.9$ $\left(\mathrm{C}_{\mathrm{q}}\right), 135.7\left(\mathrm{C}_{\mathrm{q}}\right), 137.7\left(\mathrm{C}_{\mathrm{q}}\right), 145.2\left(\mathrm{~d}, \mathrm{~J}=10.6 \mathrm{~Hz}, \mathrm{C}_{\mathrm{q}}\right), 151.1\left(\mathrm{~d}, \mathrm{~J}=244.6 \mathrm{~Hz}, \mathrm{C}_{\mathrm{q}} \mathrm{F}\right), 155.6$ $\left(\mathrm{C}_{\mathrm{q}}\right), 162.9\left(\mathrm{C}_{\mathrm{q}}\right), 166.2\left(\mathrm{C}_{\mathrm{q}}\right), 167.9\left(\mathrm{C}_{\mathrm{q}}\right) ;{ }^{19} \mathrm{~F}$ NMR $\left(376 \mathrm{MHz}, \mathrm{DMSO}-\mathrm{d}_{6}\right) \delta=-134.2(\mathrm{dd}$, $\mathrm{J}=12.2 \mathrm{~Hz}, 8.6 \mathrm{~Hz}$ ); HRMS (ESI-MS) $\mathrm{m} / \mathrm{z}$ calcd for $\mathrm{C}_{29} \mathrm{H}_{25} \mathrm{ClFN}_{4} \mathrm{O}_{7} \mathrm{~S}[\mathrm{M}+\mathrm{H}]^{+}:$627.1117, found: $627.1102 ; \mathrm{Rf}$ (petroleum ether $/ \mathrm{EtOAc}=3 / 7) 0.66$.

2-(2-Chloro-4-(((5-((1-(4-chlorobenzoyl)-5-methoxy-2-methyl-1H-indol-3-yl)methyl)-1, 3,4-oxadiazol-2-yl)thio)methyl)phenoxy)ethyl nitrate (8c): pale yellow solid, yield 78\%, m.p. 95-97 ${ }^{\circ} \mathrm{C} ;{ }^{1} \mathrm{H}$ NMR $\left(400 \mathrm{MHz}, \mathrm{DMSO}-\mathrm{d}_{6}\right) \delta=2.28(\mathrm{~s}, 3 \mathrm{H}), 3.73(\mathrm{~s}, 3 \mathrm{H}), 4.29-4.37(\mathrm{~m}, 2 \mathrm{H}), 4.35$ $(\mathrm{s}, 2 \mathrm{H}), 4.38(\mathrm{~s}, 2 \mathrm{H}), 4.84-4.92(\mathrm{~m}, 2 \mathrm{H}), 6.74(\mathrm{dd}, \mathrm{J}=9.0 \mathrm{~Hz}, 2.5 \mathrm{~Hz}, 1 \mathrm{H}), 6.92(\mathrm{~d}, \mathrm{~J}=9.0 \mathrm{~Hz}$, $1 \mathrm{H}), 6.99(\mathrm{~d}, \mathrm{~J}=8.5 \mathrm{~Hz}, 1 \mathrm{H}), 7.08(\mathrm{~d}, \mathrm{~J}=2.5 \mathrm{~Hz}, 1 \mathrm{H}), 7.23(\mathrm{dd}, \mathrm{J}=8.5 \mathrm{~Hz}, 2.2 \mathrm{~Hz}, 1 \mathrm{H}), 7.47$ $(\mathrm{d}, \mathrm{J}=2.2 \mathrm{~Hz}, 1 \mathrm{H}), 7.59-7.75(\mathrm{~m}, 4 \mathrm{H}) ;{ }^{13} \mathrm{C}$ NMR $\left(101 \mathrm{MHz}, \mathrm{DMSO}-\mathrm{d}_{6}\right) \delta=13.1\left(\mathrm{CH}_{3}\right), 20.0$ $\left(\mathrm{CH}_{2}\right), 34.7\left(\mathrm{CH}_{2}\right), 55.4\left(\mathrm{CH}_{3}\right), 65.4\left(\mathrm{CH}_{2}\right), 71.5\left(\mathrm{CH}_{2}\right), 101.5\left(\mathrm{CH}_{\mathrm{Ar}}\right), 111.6\left(\mathrm{CH}_{\mathrm{Ar}}\right), 111.8\left(\mathrm{C}_{\mathrm{q}}\right)$, $113.8\left(\mathrm{CH}_{\mathrm{Ar}}\right), 114.7\left(\mathrm{CH}_{\mathrm{Ar}}\right), 121.2\left(\mathrm{C}_{\mathrm{q}}\right), 128.9\left(\mathrm{CH}_{\mathrm{Ar}}\right), 129.0\left(2 \mathrm{CH}_{\mathrm{Ar}}\right), 129.9\left(\mathrm{C}_{\mathrm{q}}\right), 130.3\left(\mathrm{C}_{\mathrm{q}}\right)$, $130.5\left(\mathrm{CH}_{\mathrm{Ar}}\right), 130.6\left(\mathrm{C}_{\mathrm{q}}\right), 131.2\left(2 \mathrm{CH}_{\mathrm{Ar}}\right), 133.9\left(\mathrm{C}_{\mathrm{q}}\right), 135.7\left(\mathrm{C}_{\mathrm{q}}\right), 137.8\left(\mathrm{C}_{\mathrm{q}}\right), 152.6\left(\mathrm{C}_{\mathrm{q}}\right), 155.6$ 
$\left(\mathrm{C}_{\mathrm{q}}\right), 162.9\left(\mathrm{C}_{\mathrm{q}}\right), 166.2\left(\mathrm{C}_{\mathrm{q}}\right), 167.9\left(\mathrm{C}_{\mathrm{q}}\right)$; HRMS (ESI-MS) m/z calcd for $\mathrm{C}_{29} \mathrm{H}_{25} \mathrm{Cl}_{2} \mathrm{~N}_{4} \mathrm{O}_{7} \mathrm{~S}$ $[\mathrm{M}+\mathrm{H}]^{+}:$643.0821, found: 643.0816; Rf (petroleum ether $\left./ \mathrm{EtOAc}=4 / 6\right) 0.56$.

2-(2,6-Dichloro-4-(((5-((1-(4-chlorobenzoyl)-5-methoxy-2-methyl-1H-indol-3-yl)methyl)1,3,4-oxadiazol-2-yl)thio)methyl)phenoxy)ethyl nitrate (8d): pale yellow solid, yield 89\%, m.p. $125-127^{\circ} \mathrm{C} ;{ }^{1} \mathrm{H}$ NMR $\left(250 \mathrm{MHz}, \mathrm{DMSO}-\mathrm{d}_{6}\right) \delta=2.28(\mathrm{~s}, 3 \mathrm{H}), 3.73(\mathrm{~s}, 3 \mathrm{H}), 4.22-4.32(\mathrm{~m}, 2 \mathrm{H})$, $4.35(\mathrm{~s}, 2 \mathrm{H}), 4.42(\mathrm{~s}, 2 \mathrm{H}), 4.80-4.91(\mathrm{~m}, 2 \mathrm{H}), 6.72(\mathrm{dd}, \mathrm{J}=9.0 \mathrm{~Hz}, 2.5 \mathrm{~Hz}, 1 \mathrm{H}), 6.91(\mathrm{dd}, \mathrm{J}=9.0 \mathrm{~Hz}$, $0.5 \mathrm{~Hz}, 1 \mathrm{H}), 7.07(\mathrm{dd}, \mathrm{J}=2.5 \mathrm{~Hz}, 0.5 \mathrm{~Hz}, 1 \mathrm{H}), 7.54(\mathrm{~s}, 2 \mathrm{H}), 7.59-7.74(\mathrm{~m}, 4 \mathrm{H}) ;{ }^{13} \mathrm{C} \mathrm{NMR}(101 \mathrm{MHz}$, DMSO-d $\left.\mathrm{d}_{6}\right) \delta=13.1\left(\mathrm{CH}_{3}\right), 20.0\left(\mathrm{CH}_{2}\right), 34.0\left(\mathrm{CH}_{2}\right), 55.4\left(\mathrm{CH}_{3}\right), 69.4\left(\mathrm{CH}_{2}\right), 72.4\left(\mathrm{CH}_{2}\right), 101.5$ $\left(\mathrm{CH}_{\mathrm{Ar}}\right), 111.6\left(\mathrm{CH}_{\mathrm{Ar}}\right), 111.8\left(\mathrm{C}_{\mathrm{q}}\right), 114.7\left(\mathrm{CH}_{\mathrm{Ar}}\right), 128.0\left(2 \mathrm{C}_{\mathrm{q}}\right), 129.0\left(2 \mathrm{CH}_{\mathrm{Ar}}\right), 129.7\left(2 \mathrm{CH}_{\mathrm{Ar}}\right)$, $129.9\left(\mathrm{C}_{\mathrm{q}}\right), 130.2\left(\mathrm{C}_{\mathrm{q}}\right), 131.2\left(2 \mathrm{CH}_{\mathrm{Ar}}\right), 133.9\left(\mathrm{C}_{\mathrm{q}}\right), 135.7\left(\mathrm{C}_{\mathrm{q}}\right), 135.7\left(\mathrm{C}_{\mathrm{q}}\right), 137.7\left(\mathrm{C}_{\mathrm{q}}\right), 149.4\left(\mathrm{C}_{\mathrm{q}}\right)$, $155.6\left(\mathrm{C}_{\mathrm{q}}\right), 162.8\left(\mathrm{C}_{\mathrm{q}}\right), 166.3\left(\mathrm{C}_{\mathrm{q}}\right), 167.8\left(\mathrm{C}_{\mathrm{q}}\right)$; HRMS (ESI-MS) $\mathrm{m} / \mathrm{z}$ calcd for $\mathrm{C}_{29} \mathrm{H}_{24} \mathrm{Cl}_{3} \mathrm{~N}_{4} \mathrm{O}_{7} \mathrm{~S}$ $[\mathrm{M}+\mathrm{H}]^{+}:$677.0431, found: 677.0723; Rf (petroleum ether/EtOAc = 5/5) 0.49 .

2-(4-(((5-((1-(4-Chlorobenzoyl)-5-methoxy-2-methyl-1H-indol-3-yl)methyl)-1,3,4oxadiazol-2-yl)thio)methyl)-2-nitrophenoxy)ethyl nitrate (8e): pale yellow solid, yield $93 \%$, m.p. $92-94{ }^{\circ} \mathrm{C} ; .{ }^{1} \mathrm{H}$ NMR $\left(400 \mathrm{MHz}, \mathrm{DMSO}_{6}\right) \delta=2.27(\mathrm{~s}, 3 \mathrm{H}), 3.73(\mathrm{~s}, 3 \mathrm{H}), 4.35(\mathrm{~s}$, $2 \mathrm{H}), 4.39-4.51(\mathrm{~m}, 2 \mathrm{H}), 4.47(\mathrm{~s}, 2 \mathrm{H}), 4.78-4.95(\mathrm{~m}, 2 \mathrm{H}), 6.73(\mathrm{dd}, \mathrm{J}=9.0 \mathrm{~Hz}, 2.5 \mathrm{~Hz}, 1 \mathrm{H})$, $6.91(\mathrm{~d}, \mathrm{~J}=9.0 \mathrm{~Hz}, 1 \mathrm{H}), 7.06(\mathrm{~d}, \mathrm{~J}=2.5 \mathrm{~Hz}, 1 \mathrm{H}), 7.24(\mathrm{~d}, \mathrm{~J}=8.7 \mathrm{~Hz}, 1 \mathrm{H}), 7.55-7.74(\mathrm{~m}$, $5 \mathrm{H}), 7.96(\mathrm{~d}, \mathrm{~J}=2.3 \mathrm{~Hz}, 1 \mathrm{H}) ;{ }^{13} \mathrm{C}$ NMR $\left(101 \mathrm{MHz}, \mathrm{DMSO}-\mathrm{d}_{6}\right) \delta=13.0\left(\mathrm{CH}_{3}\right), 20.0\left(\mathrm{CH}_{2}\right)$, 34.2 $\left(\mathrm{CH}_{2}\right), 55.4\left(\mathrm{CH}_{3}\right), 66.0\left(\mathrm{CH}_{2}\right), 71.3\left(\mathrm{CH}_{2}\right), 101.5\left(\mathrm{CH}_{\mathrm{Ar}}\right), 111.6\left(\mathrm{CH}_{\mathrm{Ar}}\right), 111.8\left(\mathrm{C}_{\mathrm{q}}\right)$, 114.7 $\left(\mathrm{CH}_{\mathrm{Ar}}\right), 115.4\left(\mathrm{CH}_{\mathrm{Ar}}\right), 125.5\left(\mathrm{CH}_{\mathrm{Ar}}\right), 129.0\left(2 \mathrm{CH}_{\mathrm{Ar}}\right), 129.9\left(\mathrm{C}_{\mathrm{q}}\right), 130.1\left(\mathrm{C}_{\mathrm{q}}\right), 130.2\left(\mathrm{C}_{\mathrm{q}}\right)$, $131.2\left(2 \mathrm{CH}_{\mathrm{Ar}}\right), 133.9\left(\mathrm{C}_{\mathrm{q}}\right), 134.9\left(\mathrm{CH}_{\mathrm{Ar}}\right), 135.7\left(\mathrm{C}_{\mathrm{q}}\right), 137.7\left(\mathrm{C}_{\mathrm{q}}\right), 139.0\left(\mathrm{C}_{\mathrm{q}}\right), 150.0\left(\mathrm{C}_{\mathrm{q}}\right), 155.6$ $\left(\mathrm{C}_{\mathrm{q}}\right), 162.8\left(\mathrm{C}_{\mathrm{q}}\right), 166.2\left(\mathrm{C}_{\mathrm{q}}\right), 167.9\left(\mathrm{C}_{\mathrm{q}}\right)$; HRMS (ESI-MS) $\mathrm{m} / \mathrm{z}$ calcd for $\mathrm{C}_{29} \mathrm{H}_{25} \mathrm{ClN}_{5} \mathrm{O}_{9} \mathrm{~S}$ $[\mathrm{M}+\mathrm{H}]^{+}$: 654.1062, found: 654.1053; $\mathrm{Rf}\left(\mathrm{CH}_{2} \mathrm{Cl}_{2} / \mathrm{CH}_{3} \mathrm{OH}=9.8 / 0.2\right) 0.73$.

2-(4-(((5-((1-(4-Chlorobenzoyl)-5-methoxy-2-methyl-1H-indol-3-yl)methyl)-1,3,4oxadiazol-2-yl)thio)methyl)-2-methoxyphenoxy)ethyl nitrate (8f): pale yellow solid, yield 83\%, m.p. 95-97 ${ }^{\circ} \mathrm{C} ;{ }^{1} \mathrm{H}$ NMR $\left(250 \mathrm{MHz}, \mathrm{DMSO}-\mathrm{d}_{6}\right) \delta=2.29(\mathrm{~s}, 3 \mathrm{H}), 3.69(\mathrm{~s}, 3 \mathrm{H})$, $3.74(\mathrm{~s}, 3 \mathrm{H}), 4.14-4.27(\mathrm{~m}, 2 \mathrm{H}), 4.36(\mathrm{~s}, 2 \mathrm{H}), 4.38(\mathrm{~s}, 2 \mathrm{H}), 4.76-4.92(\mathrm{~m}, 2 \mathrm{H}), 6.74(\mathrm{dd}$, $\mathrm{J}=9.0 \mathrm{~Hz}, 2.5 \mathrm{~Hz}, 1 \mathrm{H}), 6.78(\mathrm{~d}, \mathrm{~J}=1.1 \mathrm{~Hz}, 2 \mathrm{H}), 6.93(\mathrm{dd}, \mathrm{J}=9.0 \mathrm{~Hz}, 0.5 \mathrm{~Hz}, 1 \mathrm{H}), 7.03$ $(\mathrm{d}, \mathrm{J}=1.2 \mathrm{~Hz}, 1 \mathrm{H}), 7.08(\mathrm{~d}, \mathrm{~J}=2.5 \mathrm{~Hz}, 1 \mathrm{H}), 7.58-7.73(\mathrm{~m}, 4 \mathrm{H}) ;{ }^{13} \mathrm{C}$ NMR $(101 \mathrm{MHz}$, DMSO-d $\left.{ }_{6}\right) \delta=13.1\left(\mathrm{CH}_{3}\right), 20.0\left(\mathrm{CH}_{2}\right), 35.9\left(\mathrm{CH}_{2}\right), 55.4\left(\mathrm{CH}_{3}\right), 55.4\left(\mathrm{CH}_{3}\right), 64.9\left(\mathrm{CH}_{2}\right), 72.0$ $\left(\mathrm{CH}_{2}\right), 101.5\left(\mathrm{CH}_{\mathrm{Ar}}\right), 111.6\left(\mathrm{CH}_{\mathrm{Ar}}\right), 111.9\left(\mathrm{C}_{\mathrm{q}}\right), 113.0\left(\mathrm{CH}_{\mathrm{Ar}}\right), 113.5\left(\mathrm{CH}_{\mathrm{Ar}}\right), 114.7\left(\mathrm{CH}_{\mathrm{Ar}}\right)$, $121.2\left(\mathrm{CH}_{\mathrm{Ar}}\right), 129.0\left(2 \mathrm{CH}_{\mathrm{Ar}}\right), 129.6\left(\mathrm{C}_{\mathrm{q}}\right), 129.9\left(\mathrm{C}_{\mathrm{q}}\right), 130.3\left(\mathrm{C}_{\mathrm{q}}\right), 131.2\left(2 \mathrm{CH}_{\mathrm{Ar}}\right), 133.9\left(\mathrm{C}_{\mathrm{q}}\right)$, $135.7\left(\mathrm{C}_{\mathrm{q}}\right), 137.7\left(\mathrm{C}_{\mathrm{q}}\right), 146.8\left(\mathrm{C}_{\mathrm{q}}\right), 148.9\left(\mathrm{C}_{\mathrm{q}}\right), 155.6\left(\mathrm{C}_{\mathrm{q}}\right), 163.1\left(\mathrm{C}_{\mathrm{q}}\right), 166.1\left(\mathrm{C}_{\mathrm{q}}\right), 167.9\left(\mathrm{C}_{\mathrm{q}}\right)$; HRMS (ESI-MS) $\mathrm{m} / \mathrm{z}$ calcd for $\mathrm{C}_{30} \mathrm{H}_{28} \mathrm{ClN}_{4} \mathrm{O}_{8} \mathrm{~S}[\mathrm{M}+\mathrm{H}]^{+}:$639.1316, found: 639.1311; Rf (petroleum ether $/ \mathrm{EtOAc}=4 / 6) 0.66$.

2-(4-(((5-((1-(4-Chlorobenzoyl)-5-methoxy-2-methyl-1H-indol-3-yl)methyl)-1,3,4oxadiazol-2-yl)thio)methyl)-2-ethoxyphenoxy)ethyl nitrate (8g): pale yellow solid, yield $91 \%$, m.p. $122-124{ }^{\circ} \mathrm{C} ;{ }^{1} \mathrm{H}$ NMR $\left(250 \mathrm{MHz}, \mathrm{DMSO}_{-} \mathrm{d}_{6}\right) \delta=1.27(\mathrm{t}, \mathrm{J}=6.9 \mathrm{~Hz}, 3 \mathrm{H}), 2.29(\mathrm{~s}$, $3 \mathrm{H}), 3.74(\mathrm{~s}, 3 \mathrm{H}), 3.94(\mathrm{q}, \mathrm{J}=6.9 \mathrm{~Hz}, 2 \mathrm{H}), 4.14-4.27(\mathrm{~m}, 2 \mathrm{H}), 4.36(\mathrm{~s}, 2 \mathrm{H}), 4.37(\mathrm{~s}, 2 \mathrm{H}), 4.75-$ $4.91(\mathrm{~m}, 2 \mathrm{H}), 6.74(\mathrm{dd}, \mathrm{J}=9.0 \mathrm{~Hz}, 2.5 \mathrm{~Hz}, 1 \mathrm{H}), 6.79(\mathrm{~d}, \mathrm{~J}=1.1 \mathrm{~Hz}, 2 \mathrm{H}), 6.93(\mathrm{dd}, \mathrm{J}=9.0 \mathrm{~Hz}$, $0.5 \mathrm{~Hz}, 1 \mathrm{H}), 7.02(\mathrm{~d}, \mathrm{~J}=1.1 \mathrm{~Hz}, 1 \mathrm{H}), 7.08(\mathrm{dd}, \mathrm{J}=2.5 \mathrm{~Hz}, 0.5 \mathrm{~Hz}, 1 \mathrm{H}), 7.57-7.73(\mathrm{~m}, 4 \mathrm{H})$; ${ }^{13} \mathrm{C}$ NMR $\left(101 \mathrm{MHz}, \mathrm{DMSO}-\mathrm{d}_{6}\right) \delta=13.1\left(\mathrm{CH}_{3}\right), 14.6\left(\mathrm{CH}_{3}\right), 20.0\left(\mathrm{CH}_{2}\right), 35.8\left(\mathrm{CH}_{2}\right), 55.4$ $\left(\mathrm{CH}_{3}\right), 63.9\left(\mathrm{CH}_{2}\right), 65.3\left(\mathrm{CH}_{2}\right), 71.9\left(\mathrm{CH}_{2}\right), 101.5\left(\mathrm{CH}_{\mathrm{Ar}}\right), 111.6\left(\mathrm{CH}_{\mathrm{Ar}}\right), 111.9\left(\mathrm{C}_{\mathrm{q}}\right), 114.3$ $\left(\mathrm{CH}_{\mathrm{Ar}}\right), 114.5\left(\mathrm{CH}_{\mathrm{Ar}}\right), 114.7\left(\mathrm{CH}_{\mathrm{Ar}}\right), 121.3\left(\mathrm{CH}_{\mathrm{Ar}}\right), 129.0\left(2 \mathrm{CH}_{\mathrm{Ar}}\right), 129.8\left(\mathrm{C}_{\mathrm{q}}\right), 129.9\left(\mathrm{C}_{\mathrm{q}}\right)$, $130.3\left(\mathrm{C}_{\mathrm{q}}\right), 131.2\left(2 \mathrm{CH}_{\mathrm{Ar}}\right), 133.9\left(\mathrm{C}_{\mathrm{q}}\right), 135.7\left(\mathrm{C}_{\mathrm{q}}\right), 137.8\left(\mathrm{C}_{\mathrm{q}}\right), 147.1\left(\mathrm{C}_{\mathrm{q}}\right), 148.3\left(\mathrm{C}_{\mathrm{q}}\right), 155.6$ $\left(\mathrm{C}_{\mathrm{q}}\right), 163.1\left(\mathrm{C}_{\mathrm{q}}\right), 166.0\left(\mathrm{C}_{\mathrm{q}}\right), 167.9\left(\mathrm{C}_{\mathrm{q}}\right)$; HRMS (ESI-MS) $\mathrm{m} / \mathrm{z}$ calcd for $\mathrm{C}_{31} \mathrm{H}_{30} \mathrm{ClN}_{4} \mathrm{O}_{8} \mathrm{~S}$ $[\mathrm{M}+\mathrm{H}]^{+}: 653.1473$, found: 653.1459; Rf (petroleum ether $/$ EtOAc $\left.=4 / 6\right) 0.58$.

2-(4-(((5-((1-(4-Chlorobenzoyl)-5-methoxy-2-methyl-1H-indol-3-yl)methyl)-1,3,4oxadiazol-2-yl)thio)methyl)-2,6-dimethoxyphenoxy)ethyl nitrate (8h): pale yellow solid, yield $91 \%$, m.p. $110-112{ }^{\circ} \mathrm{C} ;{ }^{1} \mathrm{H}$ NMR $\left(250 \mathrm{MHz}, \mathrm{DMSO}_{6} \mathrm{~d}_{6}\right) \delta=2.28(\mathrm{~s}, 3 \mathrm{H}), 3.68(\mathrm{~s}$, $6 \mathrm{H}), 3.74(\mathrm{~s}, 3 \mathrm{H}), 4.03-4.18(\mathrm{~m}, 2 \mathrm{H}), 4.36(\mathrm{~s}, 2 \mathrm{H}), 4.42(\mathrm{~s}, 2 \mathrm{H}), 4.64-4.80(\mathrm{~m}, 2 \mathrm{H}), 6.73(\mathrm{dd}$, $\mathrm{J}=9.0 \mathrm{~Hz}, 2.5 \mathrm{~Hz}, 1 \mathrm{H}), 6.73(\mathrm{~s}, 2 \mathrm{H}), 6.92(\mathrm{dd}, \mathrm{J}=9.0 \mathrm{~Hz}, 0.5 \mathrm{~Hz}, 1 \mathrm{H}), 7.09(\mathrm{~d}, \mathrm{~J}=2.3 \mathrm{~Hz}$, $1 \mathrm{H}), 7.57-7.74(\mathrm{~m}, 4 \mathrm{H}) ;{ }^{13} \mathrm{C}$ NMR $\left(101 \mathrm{MHz}, \mathrm{DMSO}_{\mathrm{d}}\right) \delta=13.1\left(\mathrm{CH}_{3}\right), 20.0\left(\mathrm{CH}_{2}\right), 36.3$ $\left(\mathrm{CH}_{2}\right), 55.4\left(\mathrm{CH}_{3}\right), 55.8\left(2 \mathrm{CH}_{3}\right), 68.5\left(\mathrm{CH}_{2}\right), 72.8\left(\mathrm{CH}_{2}\right), 101.5\left(\mathrm{CH}_{\mathrm{Ar}}\right), 106.2\left(2 \mathrm{CH}_{\mathrm{Ar}}\right), 111.5$ 
$\left(\mathrm{CH}_{\mathrm{Ar}}\right), 111.8\left(\mathrm{C}_{\mathrm{q}}\right), 114.7\left(\mathrm{CH}_{\mathrm{Ar}}\right), 129.0\left(2 \mathrm{CH}_{\mathrm{Ar}}\right), 129.9\left(\mathrm{C}_{\mathrm{q}}\right), 130.2\left(\mathrm{C}_{\mathrm{q}}\right), 131.2\left(2 \mathrm{CH}_{\mathrm{Ar}}\right)$, $132.3\left(\mathrm{C}_{\mathrm{q}}\right), 133.9\left(\mathrm{C}_{\mathrm{q}}\right), 135.3\left(\mathrm{C}_{\mathrm{q}}\right), 135.7\left(\mathrm{C}_{\mathrm{q}}\right), 137.7\left(\mathrm{C}_{\mathrm{q}}\right), 152.7\left(2 \mathrm{C}_{\mathrm{q}}\right), 155.6\left(\mathrm{C}_{\mathrm{q}}\right), 163.1\left(\mathrm{C}_{\mathrm{q}}\right)$, $166.1\left(\mathrm{C}_{\mathrm{q}}\right), 167.9\left(\mathrm{C}_{\mathrm{q}}\right)$; HRMS (ESI-MS) $\mathrm{m} / \mathrm{z}$ calcd for $\mathrm{C}_{31} \mathrm{H}_{30} \mathrm{ClN}_{4} \mathrm{O}_{9} \mathrm{~S}[\mathrm{M}+\mathrm{H}]^{+}: 669.1422$, found: $669.1411 ; \operatorname{Rf}$ (petroleum ether $/ \mathrm{EtOAc}=4 / 6) 0.57$.

2-(3-(((5-((1-(4-Chlorobenzoyl)-5-methoxy-2-methyl-1H-indol-3-yl)methyl)-1,3,4oxadiazol-2-yl)thio)methyl)phenoxy)ethyl nitrate (8i): pale yellow solid, yield $80 \%$, m.p. $98-100{ }^{\circ} \mathrm{C} ;{ }^{1} \mathrm{H}$ NMR $\left(250 \mathrm{MHz}, \mathrm{DMSO}-\mathrm{d}_{6}\right) \delta=2.28(\mathrm{~s}, 3 \mathrm{H}), 3.74(\mathrm{~s}, 3 \mathrm{H}), 4.15-$ $4.31(\mathrm{~m}, 2 \mathrm{H}), 4.35(\mathrm{~s}, 2 \mathrm{H}), 4.41(\mathrm{~s}, 2 \mathrm{H}), 4.76-4.93(\mathrm{~m}, 2 \mathrm{H}), 6.73(\mathrm{dd}, \mathrm{J}=9.0 \mathrm{~Hz}, 2.5 \mathrm{~Hz}$, $1 \mathrm{H}), 6.79-6.98(\mathrm{~m}, 2 \mathrm{H}), 6.92(\mathrm{dd}, \mathrm{J}=9.0 \mathrm{~Hz}, 0.5 \mathrm{~Hz}, 1 \mathrm{H}), 6.98-7.03(\mathrm{~m}, 1 \mathrm{H}), 7.08(\mathrm{dd}$, $\mathrm{J}=2.6 \mathrm{~Hz}, 0.5 \mathrm{~Hz}, 1 \mathrm{H}), 7.10-7.20(\mathrm{~m}, 1 \mathrm{H}), 7.58-7.73(\mathrm{~m}, 4 \mathrm{H}) ;{ }^{13} \mathrm{C}$ NMR $(101 \mathrm{MHz}$, DMSO$\left.\mathrm{d}_{6}\right) \delta=13.0\left(\mathrm{CH}_{3}\right), 20.0\left(\mathrm{CH}_{2}\right), 35.7\left(\mathrm{CH}_{2}\right), 55.4\left(\mathrm{CH}_{3}\right), 63.9\left(\mathrm{CH}_{2}\right), 71.9\left(\mathrm{CH}_{2}\right), 101.5$ $\left(\mathrm{CH}_{\mathrm{Ar}}\right), 111.6\left(\mathrm{CH}_{\mathrm{Ar}}\right), 111.9\left(\mathrm{C}_{\mathrm{q}}\right), 113.6\left(\mathrm{CH}_{\mathrm{Ar}}\right), 114.7\left(\mathrm{CH}_{\mathrm{Ar}}\right), 115.3\left(\mathrm{CH}_{\mathrm{Ar}}\right), 121.6\left(\mathrm{CH}_{\mathrm{Ar}}\right)$, 129.1 $\left(2 \mathrm{CH}_{\mathrm{Ar}}\right), 129.6\left(\mathrm{CH}_{\mathrm{Ar}}\right), 129.9\left(\mathrm{C}_{\mathrm{q}}\right), 130.2\left(\mathrm{C}_{\mathrm{q}}\right), 131.2\left(2 \mathrm{CH}_{\mathrm{Ar}}\right), 133.9\left(\mathrm{C}_{\mathrm{q}}\right), 135.7\left(\mathrm{C}_{\mathrm{q}}\right)$, $137.7\left(\mathrm{C}_{\mathrm{q}}\right), 138.1\left(\mathrm{C}_{\mathrm{q}}\right), 155.6\left(\mathrm{C}_{\mathrm{q}}\right), 157.8\left(\mathrm{C}_{\mathrm{q}}\right), 163.0\left(\mathrm{C}_{\mathrm{q}}\right), 166.1\left(\mathrm{C}_{\mathrm{q}}\right), 167.9\left(\mathrm{C}_{\mathrm{q}}\right)$. HRMS (ESIMS) $\mathrm{m} / \mathrm{z}$ calcd for $\mathrm{C}_{29} \mathrm{H}_{26} \mathrm{ClN}_{4} \mathrm{O}_{7} \mathrm{~S}[\mathrm{M}+\mathrm{H}]^{+}: 609.1211$, found: 609.1207; Rf (petroleum ether $/ \mathrm{EtOAc}=4 / 6) 0.63$.

2-(5-(((5-((1-(4-Chlorobenzoyl)-5-methoxy-2-methyl-1H-indol-3-yl)methyl)-1,3,4oxadiazol-2-yl)thio)methyl)-2-methoxyphenoxy)ethyl nitrate (8j): pale yellow solid, yield 75\%, m.p. $104-106{ }^{\circ} \mathrm{C} ;{ }^{1} \mathrm{H}$ NMR $\left(250 \mathrm{MHz}, \mathrm{DMSO}-\mathrm{d}_{6}\right) \delta=2.28(\mathrm{~s}, 3 \mathrm{H}), 3.70(\mathrm{~s}$, $3 \mathrm{H}), 3.74(\mathrm{~s}, 3 \mathrm{H}), 4.10-4.26(\mathrm{~m}, 2 \mathrm{H}), 4.36(\mathrm{~s}, 4 \mathrm{H}), 4.75-4.92(\mathrm{~m}, 2 \mathrm{H}), 6.70-6.76(\mathrm{~m}, 1 \mathrm{H})$, $6.77(\mathrm{~s}, 1 \mathrm{H}), 6.83(\mathrm{dd}, \mathrm{J}=8.3 \mathrm{~Hz}, 1.9 \mathrm{~Hz}, 1 \mathrm{H}), 6.93(\mathrm{dd}, \mathrm{J}=9.0 \mathrm{~Hz}, 0.5 \mathrm{~Hz}, 1 \mathrm{H}), 7.06(\mathrm{~d}$, $\mathrm{J}=1.9 \mathrm{~Hz}, 1 \mathrm{H}), 7.08(\mathrm{dd}, \mathrm{J}=2.5 \mathrm{~Hz}, 0.5 \mathrm{~Hz}, 1 \mathrm{H}), 7.59-7.74(\mathrm{~m}, 4 \mathrm{H}) ;{ }^{13} \mathrm{C} \mathrm{NMR}(101 \mathrm{MHz}$, DMSO-d $\left.\mathrm{d}_{6}\right) \delta=13.1\left(\mathrm{CH}_{3}\right), 20.0\left(\mathrm{CH}_{2}\right), 35.8\left(\mathrm{CH}_{2}\right), 55.4\left(\mathrm{CH}_{3}\right), 55.5\left(\mathrm{CH}_{3}\right), 65.0\left(\mathrm{CH}_{2}\right)$, $71.9\left(\mathrm{CH}_{2}\right), 101.5\left(\mathrm{CH}_{\mathrm{Ar}}\right), 111.6\left(\mathrm{CH}_{\mathrm{Ar}}\right), 111.9\left(\mathrm{C}_{\mathrm{q}}, \mathrm{CH}_{\mathrm{Ar}}\right), 114.7\left(\mathrm{CH}_{\mathrm{Ar}}\right), 114.8\left(\mathrm{CH}_{\mathrm{Ar}}\right)$, $122.3\left(\mathrm{CH}_{\mathrm{Ar}}\right), 128.5\left(\mathrm{C}_{\mathrm{q}}\right), 129.1\left(2 \mathrm{CH}_{\mathrm{Ar}}\right), 129.9\left(\mathrm{C}_{\mathrm{q}}\right), 130.3\left(\mathrm{C}_{\mathrm{q}}\right), 131.2\left(2 \mathrm{CH}_{\mathrm{Ar}}\right), 133.9\left(\mathrm{C}_{\mathrm{q}}\right)$, $135.7\left(\mathrm{C}_{\mathrm{q}}\right), 137.7\left(\mathrm{C}_{\mathrm{q}}\right), 147.0\left(\mathrm{C}_{\mathrm{q}}\right), 148.8\left(\mathrm{C}_{\mathrm{q}}\right), 155.6\left(\mathrm{C}_{\mathrm{q}}\right), 163.0\left(\mathrm{C}_{\mathrm{q}}\right), 166.0\left(\mathrm{C}_{\mathrm{q}}\right), 167.9\left(\mathrm{C}_{\mathrm{q}}\right)$; HRMS (ESI-MS) $\mathrm{m} / \mathrm{z}$ calcd for $\mathrm{C}_{30} \mathrm{H}_{28} \mathrm{ClN}_{4} \mathrm{O}_{8} \mathrm{~S}$ [M+H] ${ }^{+}$: 639.1316, found: 639.1313; Rf (petroleum ether $/ \mathrm{EtOAc}=4 / 6) 0.53$.

2-(5-(((5-((1-(4-Chlorobenzoyl)-5-methoxy-2-methyl-1H-indol-3-yl)methyl)-1,3,4oxadiazol-2-yl)thio)methyl)-2-nitrophenoxy)ethyl nitrate (8k): pale yellow solid, yield $88 \%$, m.p. $90-92{ }^{\circ} \mathrm{C} ;{ }^{1} \mathrm{H}$ NMR $\left(400 \mathrm{MHz}, \mathrm{DMSO}-\mathrm{d}_{6}\right) \delta=2.27(\mathrm{~s}, 3 \mathrm{H}), 3.72(\mathrm{~s}, 3 \mathrm{H}), 4.35(\mathrm{~s}$, $2 \mathrm{H}), 4.40-4.46(\mathrm{~m}, 2 \mathrm{H}), 4.50(\mathrm{~s}, 2 \mathrm{H}), 4.81-4.95(\mathrm{~m}, 2 \mathrm{H}), 6.73(\mathrm{dd}, \mathrm{J}=9.0 \mathrm{~Hz}, 2.5 \mathrm{~Hz}, 1 \mathrm{H})$, $6.90(\mathrm{~d}, \mathrm{~J}=9.0 \mathrm{~Hz}, 1 \mathrm{H}), 7.05(\mathrm{~d}, \mathrm{~J}=2.5 \mathrm{~Hz}, 1 \mathrm{H}), 7.09(\mathrm{dd}, \mathrm{J}=8.3 \mathrm{~Hz}, 1.6 \mathrm{~Hz}, 1 \mathrm{H}), 7.47(\mathrm{~d}$, $\mathrm{J}=1.6 \mathrm{~Hz}, 1 \mathrm{H}), 7.60-7.71(\mathrm{~m}, 4 \mathrm{H}), 7.73(\mathrm{~d}, \mathrm{~J}=8.3 \mathrm{~Hz}, 1 \mathrm{H}) ;{ }^{13} \mathrm{C}$ NMR $(101 \mathrm{MHz}$, DMSO-d 6 ) $\delta=13.0\left(\mathrm{CH}_{3}\right), 20.0\left(\mathrm{CH}_{2}\right), 35.1\left(\mathrm{CH}_{2}\right), 55.4\left(\mathrm{CH}_{3}\right), 66.0\left(\mathrm{CH}_{2}\right), 71.2\left(\mathrm{CH}_{2}\right), 101.5\left(\mathrm{CH}_{\mathrm{Ar}}\right)$, $111.5\left(\mathrm{CH}_{\mathrm{Ar}}\right), 111.8\left(\mathrm{C}_{\mathrm{q}}\right), 114.7\left(\mathrm{CH}_{\mathrm{Ar}}\right), 115.9\left(\mathrm{CH}_{\mathrm{Ar}}\right), 121.5\left(\mathrm{CH}_{\mathrm{Ar}}\right), 125.2\left(\mathrm{CH}_{\mathrm{Ar}}\right), 129.0$ $\left(2 \mathrm{CH}_{\mathrm{Ar}}\right), 129.9\left(\mathrm{C}_{\mathrm{q}}\right), 130.2\left(\mathrm{C}_{\mathrm{q}}\right), 131.2\left(2 \mathrm{CH}_{\mathrm{Ar}}\right), 133.9\left(\mathrm{C}_{\mathrm{q}}\right), 135.8\left(\mathrm{C}_{\mathrm{q}}\right), 137.8\left(\mathrm{C}_{\mathrm{q}}\right), 138.6$ $\left(\mathrm{C}_{\mathrm{q}}\right), 144.0\left(\mathrm{C}_{\mathrm{q}}\right), 150.5\left(\mathrm{C}_{\mathrm{q}}\right), 155.6\left(\mathrm{C}_{\mathrm{q}}\right), 162.6\left(\mathrm{C}_{\mathrm{q}}\right), 166.3\left(\mathrm{C}_{\mathrm{q}}\right), 167.9\left(\mathrm{C}_{\mathrm{q}}\right)$; HRMS (ESIMS) $\mathrm{m} / \mathrm{z}$ calcd for $\mathrm{C}_{29} \mathrm{H}_{25} \mathrm{ClN}_{5} \mathrm{O}_{9} \mathrm{~S}[\mathrm{M}+\mathrm{H}]^{+}: 654.1062$, found: 654.1049; Rf (petroleum ether $/ \mathrm{EtOAc}=3 / 7) 0.48$.

2-(2-Chloro-3-(((5-)(1-(4-chlorobenzoyl)-5-methoxy-2-methyl-1H-indol-3-yl)methyl)-1, 3,4-oxadiazol-2-yl)thio)methyl)-6-methoxyphenoxy)ethyl nitrate (81): pale yellow solid, yield $87 \%$, m.p. $117-119{ }^{\circ} \mathrm{C} ;{ }^{1} \mathrm{H}$ NMR $(400 \mathrm{MHz}$, DMSO-d 6 ) $\delta=2.29(\mathrm{~s}, 3 \mathrm{H}), 3.74(\mathrm{~s}, 3 \mathrm{H}), 3.76$ $(\mathrm{s}, 3 \mathrm{H}), 4.17-4.27(\mathrm{~m}, 2 \mathrm{H}), 4.37(\mathrm{~s}, 2 \mathrm{H}), 4.44(\mathrm{~s}, 2 \mathrm{H}), 4.71-4.86(\mathrm{~m}, 2 \mathrm{H}), 6.74(\mathrm{dd}, \mathrm{J}=9.0 \mathrm{~Hz}$, $2.5 \mathrm{~Hz}, 1 \mathrm{H}), 6.82(\mathrm{~d}, \mathrm{~J}=8.6 \mathrm{~Hz}, 1 \mathrm{H}), 6.93(\mathrm{~d}, \mathrm{~J}=8.9 \mathrm{~Hz}, 1 \mathrm{H}), 7.10(\mathrm{dd}, \mathrm{J}=5.5 \mathrm{~Hz}, 3.0 \mathrm{~Hz}, 2 \mathrm{H})$, 7.60-7.73 (m, 4H); ${ }^{13} \mathrm{C}$ NMR (101 MHz, DMSO-d $\left.\mathrm{d}_{6}\right) \delta=13.1\left(\mathrm{CH}_{3}\right), 20.0\left(\mathrm{CH}_{2}\right), 34.3\left(\mathrm{CH}_{2}\right)$, $55.4\left(\mathrm{CH}_{3}\right), 56.1\left(\mathrm{CH}_{3}\right), 68.8\left(\mathrm{CH}_{2}\right), 72.7\left(\mathrm{CH}_{2}\right), 101.5\left(\mathrm{CH}_{\mathrm{Ar}}\right), 111.0\left(\mathrm{CH}_{\mathrm{Ar}}\right), 111.6\left(\mathrm{CH}_{\mathrm{Ar}}\right)$, $111.8\left(\mathrm{C}_{\mathrm{q}}\right), 114.7\left(\mathrm{CH}_{\mathrm{Ar}}\right), 126.2\left(\mathrm{C}_{\mathrm{q}}\right), 126.4\left(\mathrm{CH}_{\mathrm{Ar}}\right), 127.4\left(\mathrm{C}_{\mathrm{q}}\right), 129.0\left(2 \mathrm{CH}_{\mathrm{Ar}}\right), 129.9\left(\mathrm{C}_{\mathrm{q}}\right)$, $130.2\left(\mathrm{C}_{\mathrm{q}}\right), 131.2\left(2 \mathrm{CH}_{\mathrm{Ar}}\right), 133.9\left(\mathrm{C}_{\mathrm{q}}\right), 135.8\left(\mathrm{C}_{\mathrm{q}}\right), 137.8\left(\mathrm{C}_{\mathrm{q}}\right), 143.4\left(\mathrm{C}_{\mathrm{q}}\right), 152.9\left(\mathrm{C}_{\mathrm{q}}\right), 155.6\left(\mathrm{C}_{\mathrm{q}}\right)$, $162.6\left(\mathrm{C}_{\mathrm{q}}\right), 166.3\left(\mathrm{C}_{\mathrm{q}}\right), 167.9\left(\mathrm{C}_{\mathrm{q}}\right)$; HRMS (ESI-MS) $\mathrm{m} / \mathrm{z}$ calcd for $\mathrm{C}_{30} \mathrm{H}_{27} \mathrm{Cl}_{2} \mathrm{~N}_{4} \mathrm{O}_{8} \mathrm{~S}[\mathrm{M}+\mathrm{H}]^{+}$: 673.0927, found: 673.0918; Rf (petroleum ether/EtOAc = 4/6) 0.53.

2-(2-Bromo-3-(((5-)(1-(4-chlorobenzoyl)-5-methoxy-2-methyl-1H-indol-3-yl)methyl)-1, 3,4-oxadiazol-2-yl)thio)methyl)-6-methoxyphenoxy)ethyl nitrate (8m): pale yellow solid, yield $88 \%$, m.p. $94-96{ }^{\circ} \mathrm{C} ;{ }^{1} \mathrm{H}$ NMR $\left(400 \mathrm{MHz}, \mathrm{DMSO}_{\mathrm{d}}\right) \delta=2.29(\mathrm{~s}, 3 \mathrm{H}), 3.74(\mathrm{~s}, 3 \mathrm{H}), 3.77$ 
$(\mathrm{s}, 3 \mathrm{H}), 4.16-4.24(\mathrm{~m}, 2 \mathrm{H}), 4.37(\mathrm{~s}, 2 \mathrm{H}), 4.46(\mathrm{~s}, 2 \mathrm{H}), 4.75-4.87(\mathrm{~m}, 2 \mathrm{H}), 6.74(\mathrm{dd}, \mathrm{J}=9.0 \mathrm{~Hz}$, $2.5 \mathrm{~Hz}, 1 \mathrm{H}), 6.86(\mathrm{~d}, \mathrm{~J}=8.7 \mathrm{~Hz}, 1 \mathrm{H}), 6.93(\mathrm{~d}, \mathrm{~J}=8.9 \mathrm{~Hz}, 1 \mathrm{H}), 7.10(\mathrm{~d}, \mathrm{~J}=2.5 \mathrm{~Hz}, 1 \mathrm{H}), 7.12(\mathrm{~d}$, $\mathrm{J}=8.6 \mathrm{~Hz}, 1 \mathrm{H}), 7.60-7.72(\mathrm{~m}, 4 \mathrm{H}) ;{ }^{13} \mathrm{C} \mathrm{NMR}\left(101 \mathrm{MHz}, \mathrm{DMSO}-\mathrm{d}_{6}\right) \delta=13.1\left(\mathrm{CH}_{3}\right), 20.0\left(\mathrm{CH}_{2}\right)$, $36.9\left(\mathrm{CH}_{2}\right), 55.4\left(\mathrm{CH}_{3}\right), 56.1\left(\mathrm{CH}_{3}\right), 68.6\left(\mathrm{CH}_{2}\right), 72.7\left(\mathrm{CH}_{2}\right), 101.5\left(\mathrm{CH}_{\mathrm{Ar}}\right), 111.6\left(\mathrm{CH}_{\mathrm{Ar}}\right), 111.7$ $\left(\mathrm{CH}_{\mathrm{Ar}}\right), 111.9\left(\mathrm{C}_{\mathrm{q}}\right), 114.7\left(\mathrm{CH}_{\mathrm{Ar}}\right), 119.2\left(\mathrm{C}_{\mathrm{q}}\right), 126.6\left(\mathrm{CH}_{\mathrm{Ar}}\right), 127.7\left(\mathrm{C}_{\mathrm{q}}\right), 129.0\left(2 \mathrm{CH}_{\mathrm{Ar}}\right), 129.9$ $\left(\mathrm{C}_{\mathrm{q}}\right), 130.3\left(\mathrm{C}_{\mathrm{q}}\right), 131.2\left(2 \mathrm{CH}_{\mathrm{Ar}}\right), 133.9\left(\mathrm{C}_{\mathrm{q}}\right), 135.8\left(\mathrm{C}_{\mathrm{q}}\right), 137.8\left(\mathrm{C}_{\mathrm{q}}\right), 144.5\left(\mathrm{C}_{\mathrm{q}}\right), 152.7\left(\mathrm{C}_{\mathrm{q}}\right), 155.6$ $\left(\mathrm{C}_{\mathrm{q}}\right), 162.6\left(\mathrm{C}_{\mathrm{q}}\right), 166.3\left(\mathrm{C}_{\mathrm{q}}\right), 167.9\left(\mathrm{C}_{\mathrm{q}}\right)$; HRMS (ESI-MS) $\mathrm{m} / \mathrm{z}$ calcd for $\mathrm{C}_{30} \mathrm{H}_{27} \mathrm{BrClN}_{4} \mathrm{O}_{8} \mathrm{~S}$ $[\mathrm{M}+\mathrm{H}]^{+}:$717.0421, found: 717.0409; Rf (petroleum ether $/$EtOAc $\left.=4 / 6\right) 0.55$.

2-(2-(((5-((1-(4-Chlorobenzoyl)-5-methoxy-2-methyl-1H-indol-3-yl)methyl)-1,3,4oxadiazol-2-yl)thio)methyl)phenoxy)ethyl nitrate (8n): pale yellow solid, yield 70\%, m.p. 89-91 ${ }^{\circ} \mathrm{C} ;{ }^{1} \mathrm{H}$ NMR (400 MHz, DMSO-d 6 ) $\delta=2.28(\mathrm{~s}, 3 \mathrm{H}), 3.74(\mathrm{~s}, 3 \mathrm{H}), 4.28-4.40(\mathrm{~m}$, $2 \mathrm{H}), 4.34(\mathrm{~s}, 2 \mathrm{H}), 4.35(\mathrm{~s}, 2 \mathrm{H}), 4.79-4.98(\mathrm{~m}, 2 \mathrm{H}), 6.74(\mathrm{dd}, \mathrm{J}=8.9 \mathrm{~Hz}, 2.5 \mathrm{~Hz}, 1 \mathrm{H}), 6.78$ $(\mathrm{td}, \mathrm{J}=7.5 \mathrm{~Hz}, 1.0 \mathrm{~Hz}, 1 \mathrm{H}), 6.92(\mathrm{~d}, \mathrm{~J}=8.9 \mathrm{~Hz}, 1 \mathrm{H}), 7.01(\mathrm{dd}, \mathrm{J}=8.4 \mathrm{~Hz}, 1.0 \mathrm{~Hz}, 1 \mathrm{H}), 7.08$ $(\mathrm{d}, \mathrm{J}=2.5 \mathrm{~Hz}, 1 \mathrm{H}), 7.19(\mathrm{dd}, \mathrm{J}=7.5 \mathrm{~Hz}, 1.7 \mathrm{~Hz}, 1 \mathrm{H}), 7.25(\mathrm{ddd}, \mathrm{J}=8.2 \mathrm{~Hz}, 7.4 \mathrm{~Hz}, 1.7 \mathrm{~Hz}$, 1H), 7.58-7.72 (m, 4H); ${ }^{13} \mathrm{C}$ NMR (101 MHz, DMSO-d 6$) \delta=13.1\left(\mathrm{CH}_{3}\right), 20.0\left(\mathrm{CH}_{2}\right), 31.4$ $\left(\mathrm{CH}_{2}\right), 55.4\left(\mathrm{CH}_{3}\right), 64.7\left(\mathrm{CH}_{2}\right), 71.8\left(\mathrm{CH}_{2}\right), 101.5\left(\mathrm{CH}_{\mathrm{Ar}}\right), 111.6\left(\mathrm{CH}_{\mathrm{Ar}}\right), 111.9\left(\mathrm{C}_{\mathrm{q}}\right), 112.2$ $\left(\mathrm{CH}_{\mathrm{Ar}}\right), 114.7\left(\mathrm{CH}_{\mathrm{Ar}}\right), 120.8\left(\mathrm{CH}_{\mathrm{Ar}}\right), 124.3\left(\mathrm{C}_{\mathrm{q}}\right), 129.0\left(2 \mathrm{CH}_{\mathrm{Ar}}\right), 129.6\left(\mathrm{CH}_{\mathrm{Ar}}\right), 129.9\left(\mathrm{C}_{\mathrm{q}}\right)$, $130.3\left(\mathrm{C}_{\mathrm{q}}\right), 130.3\left(\mathrm{CH}_{\mathrm{Ar}}\right), 131.2\left(2 \mathrm{CH}_{\mathrm{Ar}}\right), 133.9\left(\mathrm{C}_{\mathrm{q}}\right), 135.7\left(\mathrm{C}_{\mathrm{q}}\right), 137.7\left(\mathrm{C}_{\mathrm{q}}\right), 155.6\left(\mathrm{C}_{\mathrm{q}}\right), 155.7$ $\left(\mathrm{C}_{\mathrm{q}}\right), 163.3\left(\mathrm{C}_{\mathrm{q}}\right), 166.1\left(\mathrm{C}_{\mathrm{q}}\right), 167.9\left(\mathrm{C}_{\mathrm{q}}\right)$; HRMS (ESI-MS) $\mathrm{m} / \mathrm{z}$ calcd for $\mathrm{C}_{29} \mathrm{H}_{26} \mathrm{ClN}_{4} \mathrm{O}_{7} \mathrm{~S}$ $[\mathrm{M}+\mathrm{H}]^{+}:$609.1211, found: 609.1195; Rf (petroleum ether $/$EtOAc $\left.=4 / 6\right) 0.68$.

2-(4-Bromo-2-(((5-((1-(4-chlorobenzoyl)-5-methoxy-2-methyl-1H-indol-3-yl)methyl)-1, 3,4-oxadiazol-2-yl)thio)methyl)phenoxy)ethyl nitrate (8o): pale yellow solid, yield 87\%, m.p. 91-93 ${ }^{\circ} \mathrm{C} ;{ }^{1} \mathrm{H}$ NMR (400 MHz, DMSO-d 6 ) $\delta=2.28(\mathrm{~s}, 3 \mathrm{H}), 3.74(\mathrm{~s}, 3 \mathrm{H}), 4.26-4.41(\mathrm{~m}$, 2H), $4.34(\mathrm{~s}, 4 \mathrm{H}), 4.79-4.92(\mathrm{~m}, 2 \mathrm{H}), 6.73(\mathrm{dd}, \mathrm{J}=8.9 \mathrm{~Hz}, 2.5 \mathrm{~Hz}, 1 \mathrm{H}), 6.92(\mathrm{~d}, \mathrm{~J}=8.9 \mathrm{~Hz}$, $1 \mathrm{H}), 7.00(\mathrm{~d}, \mathrm{~J}=8.8 \mathrm{~Hz}, 1 \mathrm{H}), 7.07(\mathrm{~d}, \mathrm{~J}=2.5 \mathrm{~Hz}, 1 \mathrm{H}), 7.44(\mathrm{dd}, \mathrm{J}=8.7 \mathrm{~Hz}, 2.5 \mathrm{~Hz}, 1 \mathrm{H}), 7.50$ $(\mathrm{d}, \mathrm{J}=2.5 \mathrm{~Hz}, 1 \mathrm{H}), 7.59-7.72(\mathrm{~m}, 4 \mathrm{H}) ;{ }^{13} \mathrm{C} \mathrm{NMR}\left(101 \mathrm{MHz}, \mathrm{DMSO}-\mathrm{d}_{6}\right) \delta=13.1\left(\mathrm{CH}_{3}\right), 20.0$ $\left(\mathrm{CH}_{2}\right), 30.7\left(\mathrm{CH}_{2}\right), 55.4\left(\mathrm{CH}_{3}\right), 65.1\left(\mathrm{CH}_{2}\right), 71.7\left(\mathrm{CH}_{2}\right), 101.4\left(\mathrm{CH}_{\mathrm{Ar}}\right), 111.6\left(\mathrm{CH}_{\mathrm{Ar}}\right), 111.8$ $\left(\mathrm{C}_{\mathrm{q}}\right), 112.0\left(\mathrm{C}_{\mathrm{q}}\right), 114.4\left(\mathrm{CH}_{\mathrm{Ar}}\right), 114.7\left(\mathrm{CH}_{\mathrm{Ar}}\right), 127.1\left(\mathrm{C}_{\mathrm{q}}\right), 129.0\left(2 \mathrm{CH}_{\mathrm{Ar}}\right), 129.9\left(\mathrm{C}_{\mathrm{q}}\right), 130.2\left(\mathrm{C}_{\mathrm{q}}\right)$, 131.2 $\left(2 \mathrm{CH}_{\mathrm{Ar}}\right), 131.9\left(\mathrm{CH}_{\mathrm{Ar}}\right), 132.7\left(\mathrm{CH}_{\mathrm{Ar}}\right), 133.9\left(\mathrm{C}_{\mathrm{q}}\right), 135.7\left(\mathrm{C}_{\mathrm{q}}\right), 137.7\left(\mathrm{C}_{\mathrm{q}}\right), 155.0\left(\mathrm{C}_{\mathrm{q}}\right), 155.6$ $\left(\mathrm{C}_{\mathrm{q}}\right), 163.1\left(\mathrm{C}_{\mathrm{q}}\right), 166.2\left(\mathrm{C}_{\mathrm{q}}\right), 167.8\left(\mathrm{C}_{\mathrm{q}}\right)$; HRMS (ESI-MS) $\mathrm{m} / \mathrm{z}$ calcd for $\mathrm{C}_{29} \mathrm{H}_{25} \mathrm{BrClN}_{4} \mathrm{O}_{7} \mathrm{~S}$ $[\mathrm{M}+\mathrm{H}]^{+}:$687.0316, found: 687.0302; Rf (petroleum ether $/$EtOAc $\left.=4 / 6\right) 0.70$.

2-(4-(2-((5-((1-(4-Chlorobenzoyl)-5-methoxy-2-methyl-1H-indol-3-yl)methyl)-1,3,4oxadiazol-2-yl)thio)ethyl)phenoxy)ethyl nitrate (8p): yellow solid, yield 82\%, m.p. 92$94{ }^{\circ} \mathrm{C} ;{ }^{1} \mathrm{H}$ NMR $\left(250 \mathrm{MHz}, \mathrm{DMSO}-\mathrm{d}_{6}\right) \delta=2.29(\mathrm{~s}, 3 \mathrm{H}), 2.91(\mathrm{t}, \mathrm{J}=7.5 \mathrm{~Hz}, 2 \mathrm{H}), 3.25-3.45$ $(\mathrm{m}, 2 \mathrm{H}), 3.74(\mathrm{~s}, 3 \mathrm{H}), 4.18-4.31(\mathrm{~m}, 2 \mathrm{H}), 4.36(\mathrm{~s}, 2 \mathrm{H}), 4.79-4.93(\mathrm{~m}, 2 \mathrm{H}), 6.74(\mathrm{dd}, \mathrm{J}=9.0 \mathrm{~Hz}$, $2.5 \mathrm{~Hz}, 1 \mathrm{H}), 6.81-6.89(\mathrm{~m}, 2 \mathrm{H}), 6.92(\mathrm{dd}, \mathrm{J}=9.0 \mathrm{~Hz}, 0.5 \mathrm{~Hz}, 1 \mathrm{H}), 7.04-7.16(\mathrm{~m}, 3 \mathrm{H})$, 7.56-7.73 (m, 4H); ${ }^{13} \mathrm{C}$ NMR (101 MHz, DMSO-d 6$) \delta=13.1\left(\mathrm{CH}_{3}\right), 20.0\left(\mathrm{CH}_{2}\right), 33.4\left(\mathrm{CH}_{2}\right)$, $34.0\left(\mathrm{CH}_{2}\right), 55.4\left(\mathrm{CH}_{3}\right), 63.9\left(\mathrm{CH}_{2}\right), 72.0\left(\mathrm{CH}_{2}\right), 101.5\left(\mathrm{CH}_{\mathrm{Ar}}\right), 111.5\left(\mathrm{CH}_{\mathrm{Ar}}\right), 111.9\left(\mathrm{C}_{\mathrm{q}}\right)$, $114.4\left(2 \mathrm{CH}_{\mathrm{Ar}}\right), 114.7\left(\mathrm{CH}_{\mathrm{Ar}}\right), 129.0\left(2 \mathrm{CH}_{\mathrm{Ar}}\right), 129.7\left(2 \mathrm{CH}_{\mathrm{Ar}}\right), 129.9\left(\mathrm{C}_{\mathrm{q}}\right), 130.2\left(\mathrm{C}_{\mathrm{q}}\right), 131.2$ $\left(2 \mathrm{CH}_{\mathrm{Ar}}\right), 131.7\left(\mathrm{C}_{\mathrm{q}}\right), 133.9\left(\mathrm{C}_{\mathrm{q}}\right), 135.7\left(\mathrm{C}_{\mathrm{q}}\right), 137.7\left(\mathrm{C}_{\mathrm{q}}\right), 155.6\left(\mathrm{C}_{\mathrm{q}}\right), 156.5\left(\mathrm{C}_{\mathrm{q}}\right), 163.4\left(\mathrm{C}_{\mathrm{q}}\right)$, $165.9\left(\mathrm{C}_{\mathrm{q}}\right), 167.9\left(\mathrm{C}_{\mathrm{q}}\right)$; HRMS (ESI-MS) m/z calcd for $\mathrm{C}_{30} \mathrm{H}_{28} \mathrm{ClN}_{4} \mathrm{O}_{7} \mathrm{~S}[\mathrm{M}+\mathrm{H}]^{+}:$623.1367, found: 623.1364; Rf (petroleum ether $/$ EtOAc = 5/5) 0.63 .

\subsection{In Silico Docking Study}

In order to study the selectivity of the new NO-IND-OXDs 8a-p for COX isoenzymes (COX-1 and COX-2), AutoDock 4.2.6 software was used [82,127-129]. The results, expressed as docking score, were compared to IND, DCF and CCB, used as reference drugs.

\subsubsection{Generating the Receptor Coordinate File (RCF)}

X-ray crystallographic structures for COX-1 (pdb code: 4o1z) and COX-2 (pdb code: 3nt1)) used in this research were selected in terms of the quality of the model obtained from RCSB Protein Data Bank and were processed prior to docking. From downloaded experimental structures, the following were removed: the ligands, the water molecules, cofactors and ions that should not be included in the receptor with a text editor. After 
that, by reading the coordinates, adding charges, merging nonpolar hydrogens and assigning appropriate atom types, each receptor was converted to PDBQT format file using AutoDock 4.2.6.

\subsubsection{Generating the Ligand Coordinate File (LCF)}

The PDB coordinate files of compounds 8a-p were generated using Chimera 1.14 software. The LCF describes the ligand structure through several types of records (such as ROOT, ENDROOT, BRANCH, ENDBRANCH and TORSDOF) that are recognized by AutoDock. Each structure was energy minimized and converted to PDBQT format file. To study the interaction between a single ligand with a single receptor, with explicit calculation of affinity maps, a docking method was used. The receptors were kept rigid and the ligands were allowed to be flexible.

\subsubsection{Preparing the Grid Parameter File (GPF)}

The GPF specifies the parameters for generating the atomic affinity maps and the PDBQT files for the receptor. For COX-2 we used a grid box of $74 \times 72 \times 86$ points with a spacing of $0.375 \AA$ between grid points and the grid box center was put on $\mathrm{x}=-37.882$, $\mathrm{y}=-50.853$ and $\mathrm{z}=-21.24$. COX-1 was enclosed in a $73 \times 78 \times 82$ grid box with $0.375 \AA$ spacing and 251.00, 104.00 and 1.364 as $\mathrm{x}, \mathrm{y}$ and $\mathrm{z}$ center. The binding site of COX-1 and COX-2 ligands is respectively identified by using protein visualization software such as PyMOL, DS visualizer and Chimera 1.14.

\subsubsection{Preparing the Docking Parameter File (DPF)}

To prepare the DPF file, different parameters were selected: the ligand molecule to dock and its center and number of torsions and how many runs as well as the grid map files and the docking algorithm to use. For conformation search, the Lamarckian genetic algorithm (LGA) was applied, with the following parameters: number of runs for each docking procedure (200), number of individuals in the population (300), the maximum number of 27,000 generations simulated during each LGA run, the maximum number of evaluations at $25,000,000$, a mutation rate of 0.02 and a cross over rate of 0.80 , while the remaining docking parameters were set to default.

To identify the COX selectivity of ligands 8a-p, AutoDock 4.2.6 software was applied. After performing molecular docking simulation, the best ligand molecules were evaluated on the basis of their docking score against the COX receptors. The dockings experiments were clustered with a root mean square deviation (RMSD) of $0.5 \AA$ and were evaluated by PyMOL software. Finally, the most energetically favored orientations were selected for subsequent study.

\subsection{In Vitro Radical Scavenging Assay}

The antioxidant activity, as radical scavenging effect, of the new NO-IND-OXDs 8a-p was evaluated using 2,2-diphenyl-1-picrylhydrazyl radical (DPPH) assay with slight modification [130-132]. The results were analyzed using IND and ASP as reference drugs and vitamin $C$ as standard antioxidant.

Preparation of DPPH and test solutions. A weighed amount of DPPH $(29.8 \mathrm{mg}$, $0.076 \mathrm{mmol}$ ) was dissolved by sonication in $50 \mathrm{~mL}$ methanol of analytical grade. After $30 \mathrm{~min}$ at darkness, a sample of $10 \mathrm{~mL}$ was taken and made up to $100 \mathrm{~mL}$ with methanol. The resulting DPPH solution $(151.14 \mu \mathrm{M})$ was stored in the darkness at room temperature and used up on the day of preparation. The stock solutions $(2600 \mu \mathrm{M})$ of tested derivatives $\mathbf{8 a}-\mathbf{p}$ were prepared in DMSO, then serially diluted with methanol to obtain different concentrations $(2600 \mu \mathrm{M}, 1500 \mu \mathrm{M}, 700 \mu \mathrm{M}, 620 \mu \mathrm{M}, 530 \mu \mathrm{M}, 440 \mu \mathrm{M}$, $350 \mu \mathrm{M}, 260 \mu \mathrm{M}$ and $120 \mu \mathrm{M})$. In the same manner with tested derivatives $8 \mathbf{a}-\mathbf{p}$, the serially diluted solutions of IND and ASP were prepared. By dilution of vitamin C, freshly prepared solution $(2612 \mu \mathrm{M})$ with methanol, serially diluted solutions $(152.3 \mu \mathrm{M}$, $133.1 \mu \mathrm{M}, 112.4 \mu \mathrm{M}, 96.1 \mu \mathrm{M}, 75.3 \mu \mathrm{M}, 55.1 \mu \mathrm{M}, 40.1 \mu \mathrm{M}, 20.5 \mu \mathrm{M})$ were also prepared. 
DPPH assay procedure. First, $500 \mu \mathrm{L}$ from each sample of the tested compounds $\mathbf{8 a}-\mathbf{p}$, reference drugs (IND, ASP) and vitamin C was added to $1000 \mu \mathrm{L}$ of DPPH solution. Two blanks (blank 1: $500 \mu \mathrm{L}$ of methanol and $1000 \mu \mathrm{L}$ of DPPH and blank 2: $1500 \mu \mathrm{L}$ of methanol) were also used. The mixture was kept for $3 \mathrm{~h}$ in the darkness at room temperature and then a $270 \mu \mathrm{L}$ aliquot of each sample tube was added in a 96-well plate. The absorbance was then measured at $517 \mathrm{~nm}$ using Tecan Sunrise Remote Microplate Reader TW/ML-Abbott F039306. All tests were performed in quadruplicate.

To calculate the DPPH radical-inhibiting capacity (scavenging activity, \%), the following formula was used $[133,134]$ :

$$
\text { Inhibition (Scavenging activity) } \%=\left[\left(\mathrm{A}_{\mathrm{CS}}-\mathrm{A}_{\mathrm{S}}\right) / \mathrm{A}_{\mathrm{CS}}\right] \times 100
$$

where: $A_{s}$ is the difference between the absorbance of tested sample and blank 2 and $A_{c s}$ is the difference between absorbance of blank 1 and blank 2 .

The $\mathrm{IC}_{50}\left(\mathrm{f}_{(\mathrm{x})}=50\right)$ of each tested compound was calculated by plotting the inhibition ratios $\left(f_{(x)}\right)$ against the sample concentration $(x)$. The results for each experiment were represented by a dose-response curve and two types of regression lines $(f(x))$ were used: a sigmoid curve and a quadratic line.

The DPPH radical scavenging activity of each tested compound was also expressed as the vitamin $C$ equivalent antioxidant capacity (CEAC), which was calculated using the following formula $[135,136]$ :

$$
\left.\mathrm{CEAC}=\mathrm{IC}_{50(\text { vit }} \cdot \mathrm{C}\right) / \mathrm{IC}_{50}(\text { sample })
$$

where: $\mathrm{IC}_{50 \text { (sample) }}$ is concentration of tested compounds $\mathbf{8} \mathbf{a}-\mathbf{p}$ and reference drugs, respectively, necessary for $50 \%$ inhibition and $\left.\mathrm{IC}_{50(\mathrm{vit}} \cdot \mathrm{C}\right)$ is the concentration of vitamin $\mathrm{C}$ needed for $50 \%$ inhibition. The higher CEAC value means higher DPPH radical scavenging activity.

\subsection{Thermal Denaturation of Serum Proteins}

The anti-inflammatory effects of the new NO-IND-OXZs 8a-p were predicted by a modified Mizushima's test $[87,88]$. The test consists of the denaturing effect on specific proteins and assures a significant correlation between the in vitro and in vivo effects. A solution of bovine serum albumin (BSA) $0.2 \%$ in $0.9 \% \mathrm{NaCl} / \mathrm{DMSO}=6 / 4$ was used. Two controls (positive and negative), as well as reference drugs (IND, ASP), were also used. The positive control consisted of the action of $0.1 \mathrm{M}$ hydrochloric acid, as denaturing agent, in $0.9 \% \mathrm{NaCl}$, on the $0.2 \%$ BSA solution while the negative control consisted of untreated $0.2 \%$ BSA solution. Each sample (8a-p, IND, ASP) was tested at different concentrations $(20 \mu \mathrm{M}, 50 \mu \mathrm{M}$ and $100 \mu \mathrm{M})$. The test samples and controls were incubated at $38{ }^{\circ} \mathrm{C}$ for $5 \mathrm{~h}$. The degree of denaturation of BSA was quantified based on absorbance value, measured at $450 \mathrm{~nm}$, using Tecan Sunrise Remote Microplate Reader TW/ML-Abbott F039306. All tests were performed in quadruplicate. The maximum value of the absorbance at $450 \mathrm{~nm}$ of the positive control was considered as the $100 \%$ effect. The results were expressed as averages of the percentage values (\% effect) and were plotted versus the concentration of tested sample.

\subsection{In Vitro Nitric Oxide Release Measurement}

To assess the capacity of new NO-IND-OXDs $\mathbf{8} \mathbf{a}-\mathbf{p}$ to to release the nitric oxide (NO), the Griess colorimetric method was applied $[137,138]$. The Griess reagents consist of $0.34 \%$ (wt/v) N-(1-naphthyl)ethylenediamine (NED) solution in DMSO, 3.4\% (wt/v) sulfanilamide (SULF) in 10\% (wt/v) phosphoric acid and a mixture between $3.4 \%(\mathrm{wt} / \mathrm{v})$ SULF and $1 \%(\mathrm{wt} / \mathrm{v})$ mercuric chloride $\left(\mathrm{SULF}-\mathrm{HgCl}_{2}\right)$ in $10 \%(\mathrm{wt} / \mathrm{v})$ phosphoric acid. The $\mathrm{NO}$ released from the sample was spontaneously oxidized to $\mathrm{NO}_{2}{ }^{-}$, which subsequently reacted with the Griess reagents to form an azo dye. As reference NO donors, S-nitroso$\mathrm{N}$-acetyl-penicillamine (SNAP), sodium nitropruside (SNP) and nitroglycerine (NTG) were used. 
The experiments were performed in neutral (phosphate buffer solution-PBS) and acidic (hydrochloric acid solution- $\mathrm{HCl}$ ) experimental conditions, in the presence or absence of L-glutathione (GSH): PBS (pH 7.5), PBS-GSH (pH 7.51), HCl (pH 1.55), HCl-GSH ( $\mathrm{pH}$ 1.56).

Preparation of sodium nitrite and test solutions

A fresh sodium nitrite stock aqueous solution $(0.1 \mathrm{M})$ was prepared and was standardized according to the procedure reported in the European Pharmacopoeia (real molarity $=0.0998$, molarity factor $=0.998)$ [139]. Then, the stock solution was serially diluted with $\mathrm{MeOH} / \mathrm{H}_{2} \mathrm{O}=1 / 1(\mathrm{v} / \mathrm{v})$ mixture obtaining different concentrations $(100 \mu \mathrm{M}, 50 \mu \mathrm{M}, 25 \mu \mathrm{M}$, $12.50 \mu \mathrm{M}, 6.25 \mu \mathrm{M}, 3.125 \mu \mathrm{M}, 1.56 \mu \mathrm{M}$ and $0.78 \mu \mathrm{M})$. The tested compounds (8a-p) and reference NO donors (SNAP, SNP, NTG) were dissolved in DMSO and water, respectively, to afford a stock solution of $2600 \mu \mathrm{M}$.

The preparation of nitrite standard curve

An aliquot of $170 \mu \mathrm{L}$ sodium nitrite solution (in the range of $0.78-100 \mu \mathrm{M}$ ) was added to $50 \mu \mathrm{L}$ solution of SULF in a 96-well plate. After $10 \mathrm{~min}, 50 \mu \mathrm{L}$ of NED solution was added and then after another $20 \mathrm{~min}$, the absorbance at $540 \mathrm{~nm}$ of the formed pink-red azo dye was measured. A blank sample (containing $170 \mu \mathrm{L}$ PBS, $50 \mu \mathrm{L}$ SULF and $50 \mu \mathrm{L}$ NED) was prepared under similar conditions. All tests were performed in quintuplicate and the average absorbance was calculated. The calibration curve was constructed by graphical representation of the mean absorbance value in relation to the corresponding concentration of sodium nitrite.

NO release assay

A solution of $100 \mu \mathrm{M}$ NO-IND-OXDs 8a-p and reference NO donors (SNAP, SNP, NTG) was prepared by diluting $80 \mu \mathrm{L}$ of each stock solution $(2600 \mu \mathrm{M})$ with $2 \mathrm{~mL}$ of PBS, PBS-GSH, $\mathrm{HCl}$ and $\mathrm{HCl}-\mathrm{GSH}$. These above solutions were kept at $37-38^{\circ} \mathrm{C}$ for $120 \mathrm{~min}$; after that, an aliquot of $170 \mu \mathrm{L}$ of each solution was measured and added to $50 \mu \mathrm{L}$ of SULF and SULF- $\mathrm{HgCl}_{2}$, respectively, in a 96-well plate. After $10 \mathrm{~min}, 50 \mu \mathrm{L}$ of NED solution was added and after another $20 \mathrm{~min}$, the absorbance at $540 \mathrm{~nm}$ of the formed pink-red azo dye was measured. A blank sample (containing $170 \mu \mathrm{L}$ PBS/PBS-GSH/HCl/HCl-GSH, $50 \mu \mathrm{L}$ $\mathrm{SULF} / \mathrm{SULF}-\mathrm{HgCl}_{2}$ and $50 \mu \mathrm{L}$ NED) was prepared under similar conditions. All tests were performed in quadruplicate. The percentage (\%) of $\mathrm{NO}$ release was calculated using the following formula [137]:

$$
\% \mathrm{NO}=\left(\mathrm{C}_{\mathrm{fNO}} \times 100\right) / \mathrm{C}_{\mathrm{t} N \mathrm{O}}
$$

where: $\mathrm{C}_{\mathrm{fNO}}$ is the found concentration and $\mathrm{C}_{\mathrm{tNO}}$ is the theoretical concentration of $\mathrm{NO}(\mu \mathrm{M})$.

\subsection{Statistical Analysis}

The results were expressed as mean value \pm standard deviation (SD) and the analysis was performed using IBM SPSS Statistics 23 for Windows. The statistical significance of the results was assessed using the one-way and two-way analysis of variance (ANOVA's test). To compare the differences among samples, the Tukey's HSD test was used. A $p$ value less than 0.05 was considered statistically significant.

\section{Conclusions}

Based on the beneficial effects of 1,3,4-oxadiazole-2-thiol scaffold and NO, new nitric oxide-releasing indomethacin derivatives with 1,3,4-oxadiazole-2-thiol scaffold (NO-INDOXDs) have been synthesized. It is known that NO is an important endogenous molecule, having a critical role in protecting the GI mucosa while 1,3,4-oxadiazole-2-thiol scaffold is associated with important pharmacological effects, including anti-inflammatory and antioxidant ones. The molecular docking study revealed that $8 \mathrm{k}, 81$ and $8 \mathrm{~m}$ are COX-2 inhibitors, more selective than celecoxib. It was noted that COX-2 selectivity is influenced by the position of nitrate ester moiety, meta and para being more favorable than ortho, as well as by the nature of the substituents from phenoxy-ethylnitrate moiety, the better influence being associated with halogens $(\mathrm{F}, \mathrm{Cl}, \mathrm{Br})$ and $\mathrm{NO}_{2}$. The tested compounds also 
showed improved radical scavenging as well as anti-inflammatory properties, the last one measured as albumin denaturation effect. While for IND and ASP, a denaturation effect of less than $5 \%$ was recorded, all the tested compounds showed a denaturation effect ranging between $20 \%$ and $40 \%$ (at $100 \mu \mathrm{M}$ ). The NO release capacity of tested compounds is strongly influenced by the position of nitrate ester moiety and by the nature of the substituents from phenoxy-ethylnitrate moiety, being in good agreement with the COX-selectivity. The results of our study, related to COX-2 in silico interaction, $\mathrm{DPPH}^{\bullet}$ radical-scavenging capacity, PBS denaturation-promoting effect and NO-releasing property, encourage us to continue our research with in vivo inflammation model assay to prove the potential effect of NO-IND-OXDs as multitarget strategy.

Supplementary Materials: The following are available online at https:/ /www.mdpi.com/article/ $10.3390 /$ ijms $22137079 /$ s1.

Author Contributions: Conceptualization, L.P., S.R., F.B. and A.S.; methodology, A.S., A.P. and A.V.F.; formal analysis, N.B. and A.P.; investigation, A.S., S.M.C. and F.G.L.; resources, A.S., S.R. and L.P.; data curation, A.S., F.G.L. and A.V.F.; writing, A.S. and S.M.C.; writing-review and editing, A.S. and F.B.; supervision, S.R. and L.P.; funding acquisition, S.R. and L.P. All authors have read and agreed to the published version of the manuscript.

Funding: This research was funded by AUF-IFA 2019-2020 grant (contract no. 28/2019), University of Medicine and Pharmacy "Grigore T. Popa" of Iasi grant (contract no. 6985/2020), the FEDER under projects Valbiocosm FEDER-FSE 2017-EX003202, CHembio FEDER-FSE 2014-2020-EX003677, Techsab FEDER-FSE 2014-2020-EX011313; the SFR neuroimagerie (SFR FED 4224), the Labex programs SYNORG (ANR-11-LABX-0029) and IRON (ANR-11-LABX-0018-01), the Ligue contre le Cancer du Grand Ouest (comités des Deux Sèvres, du Finistère, de l'Ile et Villaine, du Loir et Cher, de Loire Atlantique, du Loiret, de la Vienne).We thank the above for their financial support.

Institutional Review Board Statement: Not applicable.

Informed Consent Statement: Not applicable.

Data Availability Statement: Samples of the compounds are not available from the authors.

Conflicts of Interest: The authors declare no conflict of interest.

\section{References}

1. Świacka, K.; Michnowska, A.; Maculewicz, J.; Caban, M.; Smolarz, K. Toxic effects of NSAIDs in non-target species: A review from the perspective of the aquatic environment. Environ. Pollut. 2021, 273, 115891. [CrossRef] [PubMed]

2. Holubek, W.J. Nonsteroidal anti-inflammatory drugs. In Goldfrank's Toxicologic Emergencies; Hoffman, R.S., Nelson, L.S., Howland, M.A., Lewin, N.A., Smith, S.W., Goldfrank, L.R., Eds.; McGraw-Hill: New York, NY, USA, 2019.

3. Fullerton, J.N.; Gilroy, D.W. Resolution of inflammation: A new therapeutic frontier. Nat. Rev. Drug Discov. 2016, 15, 551-567. [CrossRef] [PubMed]

4. Medzhitov, R. Origin and physiological roles of inflammation. Nature 2008, 454, 428-435. [CrossRef] [PubMed]

5. Mollaei, M.; Abbasi, A.; Hassan, Z.M.; Pakravan, N. The intrinsic and extrinsic elements regulating inflammation. Life Sci. 2020, 260. [CrossRef]

6. Korbut, R.; Guzik, T.J. Inflammatory Mediators and Intracellular Signalling. In Nijkamp and Parnham's Principles of Immunopharmacology; Parnham, M.J., Nijkamp, F.P., Rossi, A.G., Eds.; Springer International Publishing: Cham, Switzerland, 2019 ; pp. 139-163. ISBN 978-3-030-10811-3.

7. Furman, D.; Campisi, J.; Verdin, E.; Carrera-Bastos, P.; Targ, S.; Franceschi, C.; Ferrucci, L.; Gilroy, D.W.; Fasano, A.; Miller, G.W.; et al. Chronic inflammation in the etiology of disease across the life span. Nat. Med. 2019, 25, 1822-1832. [CrossRef]

8. Tyagi, V.; Singh, V.K.; Sharma, P.K.; Singh, V. Essential oil-based nanostructures for inflammation and rheumatoid arthritis. J. Drug Deliv. Sci. Technol. 2020, 60. [CrossRef]

9. Fantini, M.C.; Guadagni, I. From inflammation to colitis-associated colorectal cancer in inflammatory bowel disease: Pathogenesis and impact of current therapies. Dig. Liver Dis. 2021. [CrossRef]

10. Díaz-Ruiz, M.; Martínez-Triguero, M.L.; López-Ruiz, A.; Fernández-de la Cruz, F.; Bañuls, C.; Hernández-Mijares, A. Metabolic disorders and inflammation are associated with familial combined hyperlipemia. Clin. Chim. Acta 2019, 490, 194-199. [CrossRef]

11. Tsalamandris, S.; Antonopoulos, A.S.; Oikonomou, E.; Papamikroulis, G.; Vogiatzi, G.; Papaioannou, S.; Deftereos, S.; Tousoulis, D. The Role of Inflammation in Diabetes: Current Concepts and Future Perspectives. Eur. Cardiol. Rev. 2019, 14, 50-59. [CrossRef] 
12. Karunakaran, D.; Turner, A.W.; Duchez, A.C.; Soubeyrand, S.; Rasheed, A.; Smyth, D.; Cook, D.P.; Nikpay, M.; Kandiah, J.W.; Pan, C.; et al. RIPK1 gene variants associate with obesity in humans and can be therapeutically silenced to reduce obesity in mice. Nat. Metab. 2020, 2, 1113-1125. [CrossRef]

13. Alfaddagh, A.; Martin, S.S.; Leucker, T.M.; Michos, E.D.; Blaha, M.J.; Lowenstein, C.J.; Jones, S.R.; Toth, P.P. Inflammation and cardiovascular disease: From mechanisms to therapeutics. Am. J. Prev. Cardiol. 2020, 4, 100130. [CrossRef]

14. Sethwala, A.M.; Goh, I.; Amerena, J. V Combating Inflammation in Cardiovascular Disease. Heart Lung Circ. 2021, 30, 197-206. [CrossRef]

15. Wyss-Coray, T.; Mucke, L. Inflammation in neurodegenerative disease-A double-edged sword. Neuron 2002, 35, 419-432. [CrossRef]

16. Kinney, J.W.; Bemiller, S.M.; Murtishaw, A.S.; Leisgang, A.M.; Salazar, A.M.; Lamb, B.T. Inflammation as a central mechanism in Alzheimer's disease. Alzheimer's Dement. Transl. Res. Clin. Interv. 2018, 4, 575-590. [CrossRef]

17. Collins, L.M.; Toulouse, A.; Connor, T.J.; Nolan, Y.M. Contributions of central and systemic inflammation to the pathophysiology of Parkinson's disease. Neuropharmacology 2012, 62, 2154-2168. [CrossRef]

18. Fishbein, A.; Hammock, B.D.; Serhan, C.N.; Panigrahy, D. Carcinogenesis: Failure of resolution of inflammation? Pharmacol. Ther. 2021, 218, 107670. [CrossRef]

19. Honnappa, C.G.; Kesavan, U.M. A concise review on advances in development of small molecule anti-inflammatory therapeutics emphasising AMPK: An emerging target. Int. J. Immunopathol. Pharmacol. 2016, 29, 562-571. [CrossRef]

20. Wang, K.; Xiao, J.; Liu, X.; Jiang, Z.; Zhan, Y.; Yin, T.; He, L.; Zhang, F.; Xing, S.; Chen, B.; et al. AICD: An integrated anti-inflammatory compounds database for drug discovery. Sci. Rep. 2019, 9, 7737. [CrossRef]

21. Arfè, A.; Scotti, L.; Varas-Lorenzo, C.; Nicotra, F.; Zambon, A.; Kollhorst, B.; Schink, T.; Garbe, E.; Herings, R.; Straatman, H.; et al. Non-steroidal anti-inflammatory drugs and risk of heart failure in four European countries: Nested case-control study. BMJ 2016, 354, i4857. [CrossRef]

22. Nunes, A.P.; Costa, I.M.; Costa, F.A. Determinants of self-medication with NSAIDs in a Portuguese community pharmacy. Pharm. Pract. 2016, 14, 648. [CrossRef]

23. Aranguren, I.; Elizondo, G.; Azparren, A. Safety considerations for NSAIDs. Drug Ther. Bull. Navarre 2016, $24,1-13$.

24. Kaur, G.; Silakari, O. Multiple target-centric strategy to tame inflammation. Future Med. Chem. 2017, 9, 1361-1376. [CrossRef]

25. Moore, N.; Duong, M.; Gulmez, S.E.; Blin, P.; Droz, C. Pharmacoepidemiology of non-steroidal anti-inflammatory drugs. Therapies 2019, 74, 271-277. [CrossRef]

26. Pirlamarla, P.; Bond, R.M. FDA labeling of NSAIDs: Review of nonsteroidal anti-inflammatory drugs in cardiovascular disease. Trends Cardiovasc. Med. 2016, 26, 675-680. [CrossRef]

27. Kangwan, N.; Park, J.M.; Hahm, K.B. Development of GI-safe NSAID. Progression from the bark of willow tree to modern pharmacology. Curr. Opin. Pharmacol. 2014, 19, 17-23. [CrossRef] [PubMed]

28. Dwivedi, A.K.; Gurjar, V.; Kumar, S.; Singh, N. Molecular basis for nonspecificity of nonsteroidal anti-inflammatory drugs (NSAIDs). Drug Discov. Today 2015, 20, 863-873. [CrossRef] [PubMed]

29. Conaghan, P.G. A turbulent decade for NSAIDs: Update on current concepts of classification, epidemiology, comparative efficacy, and toxicity. Rheumatol. Int. 2012, 32, 1491-1502. [CrossRef]

30. Wongrakpanich, S.; Wongrakpanich, A.; Melhado, K.; Rangaswami, J. A comprehensive review of non-steroidal anti-inflammatory drug use in the elderly. Aging Dis. 2018, 9, 143-150. [CrossRef]

31. Koeberle, A.; Werz, O. Multi-target approach for natural products in inflammation. Drug Discov. Today 2014, 19, 1871-1882. [CrossRef]

32. Glomb, T.; Wiatrak, B.; Gębczak, K.; Gębarowski, T.; Bodetko, D.; Czyżnikowska, Ż.; Świątek, P. New 1,3,4-Oxadiazole Derivatives of Pyridothiazine-1,1-Dioxide with Anti-Inflammatory Activity. Int. J. Mol. Sci. 2020, 21, 9122. [CrossRef]

33. Rathore, A.; Rahman, M.U.; Siddiqui, A.A.; Ali, A.; Shaharyar, M. Design and Synthesis of Benzimidazole Analogs Endowed with Oxadiazole as Selective COX-2 Inhibitor. Arch. Pharm. 2014, 347, 923-935. [CrossRef] [PubMed]

34. Banerjee, A.G.; Das, N.; Shengule, S.A.; Sharma, P.A.; Srivastava, R.S.; Shrivastava, S.K. Design, synthesis, evaluation and molecular modelling studies of some novel 5,6-diphenyl-1,2,4-triazin-3(2H)-ones bearing five-member heterocyclic moieties as potential COX-2 inhibitors: A hybrid pharmacophore approach. Bioorg. Chem. 2016, 69, 102-120. [CrossRef] [PubMed]

35. Hughes, M.N. Chemistry of Nitric Oxide and Related Species. In Methods in Enzymology; Academic Press: Cambridge, MA, USA, 2008; Volume 436, pp. 3-19, ISBN 9780123742773.

36. Procházková, D.; Wilhelmová, N.; Pavlík, M. Reactive Nitrogen Species and Nitric Oxide. In Nitric Oxide Action in Abiotic Stress Responses in Plants; Springer International Publishing: Cham, Switzerland, 2015; pp. 3-19, ISBN 9783319178042.

37. Lundberg, J.O.; Weitzberg, E. Nitric Oxide Formation From Inorganic Nitrate. In Nitric Oxide; Elsevier: Amsterdam, The Netherlands, 2017; pp. 157-171, ISBN 9780128042731.

38. Williams, D.L.H. Nitric oxide in biological systems. In Nitrosation Reactions and the Chemistry of Nitric Oxide; Elsevier: Amsterdam, The Netherlands, 2004; Volume 18, pp. 187-198.

39. Kumar, S.; Singh, R.K.; Bhardwaj, T.R. Therapeutic role of nitric oxide as emerging molecule. Biomed. Pharmacother. 2017, 85, 182-201. [CrossRef] [PubMed]

40. Sharma, J.N.; Al-Omran, A.; Parvathy, S.S. Role of nitric oxide in inflammatory diseases. Inflammopharmacology 2007, 15, 252-259. [CrossRef] 
41. Hirst, D.G.; Robson, T. Nitric Oxide Physiology and Pathology. In Methods in Molecular Biology; Humana Press: Totowa, NJ, USA, 2011; Volume 704, pp. 1-13, ISBN 9781617379642.

42. Lanas, A. Role of nitric oxide in the gastrointestinal tract. Arthritis Res. Ther. 2008, 10, S4. [CrossRef]

43. Santana, A.P.M.; Tavares, B.M.; Lucetti, L.T.; Gouveia, F.S.; Ribeiro, R.A.; Soares, P.M.G.; Sousa, E.H.S.; Lopes, L.G.F.; Medeiros, J.-V.R.; Souza, M.H.L.P. The nitric oxide donor cis-[Ru(bpy)2(SO3)NO](PF6) increases gastric mucosa protection in mice-Involvement of the soluble guanylate cyclase/KATP pathway. Nitric Oxide 2015, 45, 35-42. [CrossRef]

44. Lucas, S. The Pharmacology of Indomethacin. Headache J. Head Face Pain 2016, 56, 436-446. [CrossRef]

45. Mohamed, M.F.A.; Marzouk, A.A.; Nafady, A.; El-Gamal, D.A.; Allam, R.M.; Abuo-Rahma, G.E.D.A.; El Subbagh, H.I.; Moustafa, A.H. Design, synthesis and molecular modeling of novel aryl carboximidamides and 3-aryl-1,2,4-oxadiazoles derived from indomethacin as potent anti-inflammatory iNOS/PGE2 inhibitors. Bioorg. Chem. 2020, 105, 104439. [CrossRef]

46. Stachowicz, K. Indomethacin, a nonselective cyclooxygenase inhibitor, does not interact with MTEP in antidepressant-like activity, as opposed to imipramine in CD-1 mice. Eur. J. Pharmacol. 2020, 888, 173585. [CrossRef]

47. Siwach, A.; Verma, P.K. Therapeutic potential of oxadiazole or furadiazole containing compounds. BMC Chem. 2020, 14, 70. [CrossRef]

48. Paruch, K.; Popiołek, Ł.; Biernasiuk, A.; Hordyjewska, A.; Malm, A.; Wujec, M. Novel 3-Acetyl-2,5-disubstituted-1,3,4oxadiazolines: Synthesis and Biological Activity. Molecules 2020, 25, 5844. [CrossRef]

49. Mazumder, S.A.; Yar, M.S.; Mazumder, R.; Chakraborthy, G.S.; Ahsan, M.J.; Rahman, M.U. Updates on synthesis and biological activities of 1,3,4-oxadiazole: A review. Synth. Commun. 2017, 47, 1805-1847. [CrossRef]

50. Glomb, T.; Szymankiewicz, K.; Świątek, P. Anti-Cancer Activity of Derivatives of 1,3,4-Oxadiazole. Molecules 2018, $23,3361$. [CrossRef]

51. Boström, J.; Hogner, A.; Llinàs, A.; Wellner, E.; Plowright, A.T. Oxadiazoles in Medicinal Chemistry. J. Med. Chem. 2012, 55, 1817-1830. [CrossRef]

52. Yatam, S.; Jadav, S.S.; Gundla, R.; Gundla, K.P.; Reddy, G.M.; Ahsan, M.J.; Chimakurthy, J. Design, Synthesis and Biological Evaluation of 2 (((5-aryl-1,2,4-oxadiazol-3-yl)methyl)thio)benzo[d]oxazoles: New Antiinflammatory and Antioxidant Agents. ChemistrySelect 2018, 3, 10305-10310. [CrossRef]

53. Chawla, G.; Naaz, B.; Siddiqui, A.A. Exploring 1,3,4-Oxadiazole Scaffold for Anti-inflammatory and Analgesic Activities: A Review of Literature From 2005-2016. Mini-Rev. Med. Chem. 2018, 18, 216-233. [CrossRef]

54. Alisi, I.O.; Uzairu, A.; Abechi, S.E. Free radical scavenging mechanism of 1,3,4-oxadiazole derivatives: Thermodynamics of O-H and $\mathrm{N}-\mathrm{H}$ bond cleavage. Heliyon 2020, 6, e03683. [CrossRef]

55. Jakovljević, K.; Joksović, M.D.; Botta, B.; Jovanović, L.S.; Avdović, E.; Marković, Z.; Mihailović, V.; Andrić, M.; Trifunović, S.; Marković, V. Novel 1,3,4-thiadiazole conjugates derived from protocatechuic acid: Synthesis, antioxidant activity, and computational and electrochemical studies. ComptesRendusChim. 2019, 22, 585-598. [CrossRef]

56. Bhutani, R.; Pathak, D.P.; Kapoor, G.; Husain, A.; Iqbal, M.A. Novel hybrids of benzothiazole-1,3,4-oxadiazole-4-thiazolidinone: Synthesis, in silico ADME study, molecular docking and in vivo anti-diabetic assessment. Bioorg. Chem. 2019, 83, 6-19. [CrossRef]

57. Wang, S.; Liu, H.; Wang, X.; Lei, K.; Li, G.; Li, J.; Liu, R.; Quan, Z. Synthesis of 1,3,4-oxadiazole derivatives with anticonvulsant activity and their binding to the GABAA receptor. Eur. J. Med. Chem. 2020, 206, 112672. [CrossRef]

58. Mohan, C.D.; Anilkumar, N.C.; Rangappa, S.; Shanmugam, M.K.; Mishra, S.; Chinnathambi, A.; Alharbi, S.A.; Bhattacharjee, A.; Sethi, G.; Kumar, A.P.; et al. Novel 1,3,4-Oxadiazole Induces Anticancer Activity by Targeting NF- $\mathrm{B}$ in Hepatocellular Carcinoma Cells. Front. Oncol. 2018, 8, 1-11. [CrossRef]

59. Makane, V.B.; Krishna, V.S.; Krishna, E.V.; Shukla, M.; Mahizhaveni, B.; Misra, S.; Chopra, S.; Sriram, D.; AzgerDusthackeer, V.N.; Rode, H.B. Novel 1,3,4-oxadiazoles as antitubercular agents with limited activity against drug-resistant tuberculosis. Future Med. Chem. 2019, 11, 499-510. [CrossRef]

60. Raval, J.P.; Akhaja, T.N.; Jaspara, D.M.; Myangar, K.N.; Patel, N.H. Synthesis and in vitro antibacterial activity of new oxoethylthio1,3,4-oxadiazole derivatives. J. Saudi Chem. Soc. 2014, 18, 101-106. [CrossRef]

61. Gan, X.; Hu, D.; Chen, Z.; Wang, Y.; Song, B. Synthesis and antiviral evaluation of novel 1,3,4-oxadiazole/thiadiazole-chalcone conjugates. Bioorg. Med. Chem. Lett. 2017, 27, 4298-4301. [CrossRef]

62. Wang, S.; Qi, L.; Liu, H.; Lei, K.; Wang, X.; Liu, R. Synthesis of 1,3,4-oxadiazoles derivatives with antidepressant activity and their binding to the 5-HT 1A receptor. RSC Adv. 2020, 10, 30848-30857. [CrossRef]

63. Ajani, O.O.; Iyaye, K.T. Recent advances on oxadiazole motifs: Synthesis, reactions and biological activities. Mediterr. J. Chem. 2020, 10, 418. [CrossRef]

64. Carbone, M.; Li, Y.; Irace, C.; Mollo, E.; Castelluccio, F.; Di Pascale, A.; Cimino, G.; Santamaria, R.; Guo, Y.-W.; Gavagnin, M. Structure and Cytotoxicity of Phidianidines A and B: First Finding of 1,2,4-Oxadiazole System in a Marine Natural Product. Org. Lett. 2011, 13, 2516-2519. [CrossRef]

65. Tanaka, S.; Nelson, G.; Olson, C.A.; Buzko, O.; Higashide, W.; Shin, A.; Gonzalez, M.; Taft, J.; Patel, R.; Buta, S.; et al. An ACE2 Triple Decoy that neutralizes SARS-CoV-2 shows enhanced affinity for virus variants. Sci. Rep. 2021, 11, 12740. [CrossRef]

66. Maniak, H.; Talma, M.; Matyja, K.; Trusek, A.; Giurg, M. Synthesis and structure-activity relationship studies of hydrazidehydrazones as inhibitors of laccase from trametes versicolor. Molecules 2020, 25, 1255. [CrossRef]

67. Mysinger, M.M.; Carchia, M.; Irwin, J.J.; Shoichet, B.K. Directory of useful decoys, enhanced (DUD-E): Better ligands and decoys for better benchmarking. J. Med. Chem. 2012, 55, 6582-6594. [CrossRef] 
68. Huang, K.; Lin, M.; Kuo, T.; Chen, C.; Lin, C.; Chou, Y.; Chao, T.; Pang, Y.; Kao, H.; Huang, R.; et al. Humanized COVID-19 decoy antibody effectively blocks viral entry and prevents SARS-CoV-2 infection. EMBO Mol. Med. 2021, 13, e12828. [CrossRef] [PubMed]

69. Patai, S. The Hydroxyl Group (1971); John Wiley \& Sons, Ltd.: Chichester, UK, 1971; Volume 10, ISBN 9780470771259.

70. Chaudhari, S.S.; Akamanchi, K.G. Thionyl Chloride-Benzotriazole in Methylene Chloride: A Convenient Solution for Conversion of Alcohols and Carboxylic Acids Expeditiously into Alkyl Chlorides and Acid Chlorides by Simple Titration. Synlett 1999, 1999, 1763-1765. [CrossRef]

71. Bandgar, B.P.; Bettigeri, S.V. Efficient and Selective Halogenation of Allylic and Benzylic Alcohols under Mild Conditions. Mon. Chem. 2004, 135, 1251-1255. [CrossRef]

72. Gore, S.; Sanz García, E.; Hendrickx, P.M.S.; Gutmanas, A.; Westbrook, J.D.; Yang, H.; Feng, Z.; Baskaran, K.; Berrisford, J.M.; Hudson, B.P.; et al. Validation of Structures in the Protein Data Bank. Structure 2017, 25, 1916-1927. [CrossRef]

73. Duggan, K.C.; Walters, M.J.; Musee, J.; Harp, J.M.; Kiefer, J.R.; Oates, J.A.; Marnett, L.J. Molecular basis for cyclooxygenase inhibition by the non-steroidal anti-inflammatory drug naproxen. J. Biol. Chem. 2010, 285, 34950-34959. [CrossRef]

74. Morris, A.L.; MacArthur, M.W.; Hutchinson, E.G.; Thornton, J.M. Stereochemical quality of protein structure coordinates. Proteins Struct. Funct. Bioinform. 1992, 12, 345-364. [CrossRef]

75. Kleywegt, G.J.; Alwyn Jones, T.B.T.-M. [11] Model building and refinement practice. In Macromolecular Crystallography Part B; Academic Press: Cambridge, MA, USA, 1997; Volume 277, pp. 208-230, ISBN 0076-6879.

76. Oniga, S.; Pacureanu, L.; Stoica, C.; Palage, M.; Crăciun, A.; Rusu, L.; Crisan, E.-L.; Araniciu, C. COX Inhibition Profile and Molecular Docking Studies of Some 2-(Trimethoxyphenyl)-Thiazoles. Molecules 2017, 22, 1507. [CrossRef]

77. Kassab, S.E.; Khedr, M.A.; Ali, H.I.; Abdalla, M.M. Discovery of new indomethacin-based analogs with potentially selective cyclooxygenase-2 inhibition and observed diminishing to PGE2 activities. Eur. J. Med. Chem. 2017, 141, 306-321. [CrossRef]

78. Rowlinson, S.W.; Kiefer, J.R.; Prusakiewicz, J.J.; Pawlitz, J.L.; Kozak, K.R.; Kalgutkar, A.S.; Stallings, W.C.; Kurumbail, R.G.; Marnett, L.J. A Novel Mechanism of Cyclooxygenase-2 Inhibition Involving Interactions with Ser-530 and Tyr-385. J. Biol. Chem. 2003, 278, 45763-45769. [CrossRef]

79. Charles, D.J. Antioxidant Assays. In Antioxidant Properties of Spices, Herbs and Other Sources; Springer: New York, NY, USA, 2012; Volume 9781461443, pp. 9-38, ISBN 9781461443100.

80. González-Trujano, M.E.; Uribe-Figueroa, G.; Hidalgo-Figueroa, S.; Martínez, A.L.; Déciga-Campos, M.; Navarrete-Vazquez, G. Synthesis and antinociceptive evaluation of bioisosteres and hybrids of naproxen, ibuprofen and paracetamol. Biomed. Pharmacother. 2018, 101, 553-562. [CrossRef]

81. Hidalgo-Figueroa, S.; Estrada-Soto, S.; Ramírez-Espinosa, J.J.; Paoli, P.; Lori, G.; León-Rivera, I.; Navarrete-Vázquez, G. Synthesis and evaluation of thiazolidine-2,4-dione/benzazole derivatives as inhibitors of protein tyrosine phosphatase 1B (PTP-1B): Antihyperglycemic activity with molecular docking study. Biomed. Pharmacother. 2018, 107, 1302-1310. [CrossRef]

82. Forli, S.; Huey, R.; Pique, M.E.; Sanner, M.F.; Goodsell, D.S.; Olson, A.J. Computational protein-ligand docking and virtual drug screening with the AutoDock suite. Nat. Protoc. 2016, 11, 905-919. [CrossRef]

83. Khalilullah, H.; Ahsan, M.J.; Hedaitullah, M.; Khan, S.; Ahmed, B. 1,3,4-Oxadiazole: A Biologically Active Scaffold. Mini-Rev. Med. Chem. 2012, 12, 789-801. [CrossRef]

84. Basappa, V.C.; Penubolu, S.; Achutha, D.K.; Kariyappa, A.K. Synthesis, characterization and antioxidant activity studies of new coumarin tethered 1,3,4-oxadiazole analogues. J. Chem. Sci. 2021, 133, 55. [CrossRef]

85. Mizushima, Y.; Kobayashi, M. Interaction of anti-inflammatory drugs with serum proteins, especially with some biologically active proteins. J. Pharm. Pharmacol. 1968, 20, 169-173. [CrossRef]

86. Senthil, S.K.; Murugan, P.K.; Selvam, S.; Chandhana, J.P.; Satheesh Babu, T.G.; Kandasamy, E. Fluorescence spectroscopic analysis of heavy metal induced protein denaturation. Mater. Today Proc. 2020, 33, 2328-2330. [CrossRef]

87. Loukianov, A.S.; Syomina, T.K.; Korolev, A.M. Conformational Changes in Proteins In Vitro as a Means of Predicting the Acute Toxicities of Chemicals. Altern. Lab. Anim. 2007, 35, 123-136. [CrossRef]

88. Novillo, A.; Ekwall, B.; Castaño, A. Protein Precipitation In Vitro as a Measure of Chemical-induced Cytotoxicity: An EDIT Sub-programme. Altern. Lab. Anim. 2001, 29, 309-324. [CrossRef]

89. Pabbathi, A.; Patra, S.; Samanta, A. Structural Transformation of Bovine Serum Albumin Induced by Dimethyl Sulfoxide and Probed by Fluorescence Correlation Spectroscopy and Additional Methods. ChemPhysChem 2013, 14, 2441-2449. [CrossRef]

90. Batista, A.N.L.; Batista, J.M.; Ashton, L.; Bolzani, V.S.; Furlan, M.; Blanch, E.W. Investigation of DMSO-Induced Conformational Transitions in Human Serum Albumin Using Two-Dimensional Raman Optical Activity Spectroscopy. Chirality 2014, $26,497-501$. [CrossRef]

91. Alfonso, L.; Ai, G.; Spitale, R.C.; Bhat, G.J. Molecular targets of aspirin and cancer prevention. Br. J. Cancer 2014, 111, 61-67. [CrossRef]

92. Alfonso, L.F.; Srivenugopal, K.S.; Bhat, G.J. Does aspirin acetylate multiple cellular proteins? (Review). Mol. Med. Rep. 2009, 2, 667-671. [CrossRef]

93. Tatham, M.H.; Cole, C.; Scullion, P.; Wilkie, R.; Westwood, N.J.; Stark, L.A.; Hay, R.T. A Proteomic Approach to Analyze the Aspirin-mediated Lysine Acetylome. Mol. Cell. Proteom. 2017, 16, 310-326. [CrossRef] 
94. Gryglewski, R.J. Screening and Assessment of the Potency of Anti-Inflammatory Drugs in vitro. In Anti-Inflammatory Drugs. Handbook of Experimental Pharmacology (Continuation of Handbuch der ExperimentellenPharmakologie); Vane, J.R., Ferreira, S., Eds.; Springer: Berlin/Heidelberg, Germany, 1979; pp. 3-43.

95. Thomas, D.D.; Heinecke, J.L.; Ridnour, L.A.; Cheng, R.Y.; Kesarwala, A.H.; Switzer, C.H.; McVicar, D.W.; Roberts, D.D.; Glynn, S.; Fukuto, J.M.; et al. Signaling and stress: The redox landscape in NOS2 biology. Free Radic. Biol. Med. 2015, 87, 204-225. [CrossRef]

96. Hada, K.; Suda, A.; Asoh, K.; Tsukuda, T.; Hasegawa, M.; Sato, Y.; Ogawa, K.; Kuramoto, S.; Aoki, Y.; Shimma, N.; et al. Angiogenesis inhibitors identified by cell-based high-throughput screening: Synthesis, structure-activity relationships and biological evaluation of 3-[(E)-styryl]benzamides that specifically inhibit endothelial cell proliferation. Bioorg. Med. Chem. 2012, 20, 1442-1460. [CrossRef]

97. Ortmeyer, C.P.; Haufe, G.; Schwegmann, K.; Hermann, S.; Schäfers, M.; Börgel, F.; Wünsch, B.; Wagner, S.; Hugenberg, V. Synthesis and evaluation of a [18 F]BODIPY-labeled caspase-inhibitor. Bioorg. Med. Chem. 2017, 25, 2167-2176. [CrossRef]

98. Del Carmen Gimenez-Lopez, M.; Räisänen, M.T.; Chamberlain, T.W.; Weber, U.; Lebedeva, M.; Rance, G.A.; Briggs, G.A.D.; Pettifor, D.; Burlakov, V.; Buck, M.; et al. Functionalized Fullerenes in Self-Assembled Monolayers. Langmuir 2011, 27, 10977-10985. [CrossRef]

99. Sava, A.; Buron, F.; Routier, S.; Panainte, A.; Bibire, N.; Profire, L. New nitric oxide-releasing indomethacin derivatives with 1,3-thiazolidine-4-one scaffold: Design, synthesis, in silico and in vitro studies. Biomed. Pharmacother. 2021, 139, 111678. [CrossRef]

100. Ward, D.E.; Rhee, C.K. Chemoselective reductions with sodium borohydride. Can. J. Chem. 1989, 67, 1206-1211. [CrossRef]

101. Gisch, N.; Balzarini, J.; Meier, C. Enzymatically Activated cyclo Sal-d4T-monophosphates: The Third Generation of cyclo Sal-Pronucleotides. J. Med. Chem. 2007, 50, 1658-1667. [CrossRef]

102. Ghazy, E.; Zeyen, P.; Herp, D.; Hügle, M.; Schmidtkunz, K.; Erdmann, F.; Robaa, D.; Schmidt, M.; Morales, E.R.; Romier, C.; et al. Design, synthesis, and biological evaluation of dual targeting inhibitors of histone deacetylase 6/8 and bromodomain BRPF1. Eur. J. Med. Chem. 2020, 200, 112338. [CrossRef] [PubMed]

103. Ikegashira, K.; Ikenogami, T.; Yamasaki, T.; Oka, T.; Hase, Y.; Miyagawa, N.; Inagaki, K.; Kawahara, I.; Koga, Y.; Hashimoto, H. Optimization of an azetidine series as inhibitors of colony stimulating factor-1 receptor (CSF-1R) Type II to lead to the clinical candidate JTE-952. Bioorg. Med. Chem. Lett. 2019, 29, 873-877. [CrossRef] [PubMed]

104. Zhang, Z.; Cui, M.; Lin, C.; Liu, B.; Guo, Y. Phenyl Benzyl Ether Derivative and Preparation Method and Application Thereof. Patent Number US 2017/0037008 A1, 9 February 2017.

105. Chawla, H.M.; Shrivastava, R. Control of Conformational Flexibility in Calix[6]Arenes: Synthesis and Characterisation of Triply Bridged Calix[6]Arene-10,15-Dihydro-5 H -Tribenzo [a,d,g]Cyclononene Conjugates. J. Chem. Res. 2008, 2008, 347-353. [CrossRef]

106. Canceill, J.; Lacombe, L.; Collet, A. Analytical optical resolution of bromochlorofluoromethane by enantioselective inclusion into a tailor-made cryptophane and determination of its maximum rotation. J. Am. Chem. Soc. 1985, 107, 6993-6996. [CrossRef]

107. Imai, K.; Niigata, K.; Fujikura, T.; Hashimoto, S.; Takenaka, T. Phenylethanolamine derivative. Patent numbers US $4217305,1980$.

108. Mingdong, Y.; Bin, Z.; Wanqiang, D. Rotaxane Molecular Machine of Naphthalimide Crown Ether and Ph Meter Based on Rotaxane Molecular Machine. Patent Number CN 103539790 A, 30 September 2013.

109. Pulpoka, B.; Jamkratoke, M.; Tuntulani, T.; Ruangpornvisuti, V. Synthesis of 1,3-alternate calix[4]-cyclen-benzo-crown-6 as a hard-soft receptor. Tetrahedron Lett. 2000, 41, 9167-9171. [CrossRef]

110. Liu, X.X.; Yang, Y.T.; Wang, X.; Wang, K.Y.; Liu, J.Q.; Lei, L.; Luo, X.M.; Zhai, R.; Fu, F.H.; Wang, H.B.; et al. Design, synthesis and biological evaluation of novel $\alpha$-hederagenin derivatives with anticancer activity. Eur. J. Med. Chem. 2017, 141, 427-439. [CrossRef]

111. Chen, Y.; Sun, J.; Huang, Z.; Liao, H.; Peng, S.; Lehmann, J.; Zhang, Y. Design, synthesis and evaluation of tacrine-flurbiprofennitrate trihybrids as novel anti-Alzheimer's disease agents. Bioorg. Med. Chem. 2013, 21, 2462-2470. [CrossRef]

112. Liu, J.; Zhang, C.; Wang, H.; Zhang, L.; Jiang, Z.; Zhang, J.; Liu, Z.; Chen, H. Incorporation of nitric oxide donor into 1,3dioxyxanthones leads to synergistic anticancer activity. Eur. J. Med. Chem. 2018, 151, 158-172. [CrossRef]

113. Wey, S.-J.; Augustyniak, M.E.; Cochran, E.D.; Ellis, J.L.; Fang, X.; Garvey, D.S.; Janero, D.R.; Letts, L.G.; Martino, A.M.; Melim, T.L.; et al. Structure-Based Design, Synthesis, and Biological Evaluation of Indomethacin Derivatives as Cyclooxygenase-2 Inhibiting Nitric Oxide Donors. J. Med. Chem. 2007, 50, 6367-6382. [CrossRef]

114. Lazzarato, L.; Donnola, M.; Rolando, B.; Chegaev, K.; Marini, E.; Cena, C.; Di Stilo, A.; Fruttero, R.; Biondi, S.; Ongini, E.; et al. (Nitrooxyacyloxy)methyl Esters of Aspirin as Novel Nitric Oxide Releasing Aspirins. J. Med. Chem. 2009, 52, 5058-5068. [CrossRef]

115. Liu, W.; Liu, C.; Gong, C.; Lin, W.; Guo, C. Porphyrins containing nitric oxide donors: Synthesis and cancer cell-oriented NO release. Bioorg. Med. Chem. Lett. 2009, 19, 1647-1649. [CrossRef]

116. Nortcliffe, A.; Fleming, I.N.; Botting, N.P.; O'Hagan, D. Synthesis and anticancer properties of RGD peptides conjugated to nitric oxide releasing functional groups and abiraterone. Tetrahedron 2014, 70, 8343-8347. [CrossRef]

117. Fotopoulou, T.; Iliodromitis, E.K.; Koufaki, M.; Tsotinis, A.; Zoga, A.; Gizas, V.; Pyriochou, A.; Papapetropoulos, A.; Andreadou, I.; Kremastinos, D.T. Design and synthesis of nitrate esters of aromatic heterocyclic compounds as pharmacological preconditioning agents. Bioorg. Med. Chem. 2008, 16, 4523-4531. [CrossRef]

118. Wang, Q.; Hu, W.; Wang, S.; Pan, Z.; Tao, L.; Guo, X.; Qian, K.; Chen, C.-H.; Lee, K.-H.; Chang, J. Synthesis of new 2'-deoxy-2'fluoro-4'-azido nucleoside analogues as potent anti-HIV agents. Eur. J. Med. Chem. 2011, 46, 4178-4183. [CrossRef] 
119. Shan, Y.; Wang, J.; Si, R.; Ma, Y.; Li, J.; Zhang, Q.; Lu, W.; Zhang, J. Exploring the potential intracellular targets of vascular normalization based on active candidates. Bioorg. Chem. 2021, 108, 104551. [CrossRef]

120. Kojima, M.; Matsunaga, S. Allyl 4-Chlorophenyl Sulfone as a Versatile 1,1-Synthon for Sequential $\alpha$-Alkylation/Cobalt-Catalyzed Allylic Substitution. Synthesis 2020, 52, 1934-1946. [CrossRef]

121. Gurenko, A.O.; Khutova, B.M.; Klyuchko, S.V.; Vasilenko, A.N.; Brovarets, V.S. Synthesis of Novel Pyrazolo[3,4-d][1,2,3]Triazines. Chem. Heterocycl. Compd. 2014, 50, 528-536. [CrossRef]

122. Chen, J.; Wei, C.; Wu, S.; Luo, Y.; Wu, R.; Hu, D.; Song, B. Novel 1,3,4-oxadiazole thioether derivatives containing flexible-chain moiety: Design, synthesis, nematocidal activities, and pesticide-likeness analysis. Bioorg. Med. Chem. Lett. 2020, $30,127028$. [CrossRef]

123. Narella, S.G.; Shaik, M.G.; Mohammed, A.; Alvala, M.; Angeli, A.; Supuran, C.T. Synthesis and biological evaluation of coumarin-1,3,4-oxadiazole hybrids as selective carbonic anhydrase IX and XII inhibitors. Bioorg. Chem. 2019, 87, 765-772. [CrossRef]

124. Gulnaz, Z.A.R.; Mohammed, Y.H.E.; Khanum, S.A. Design, synthesis and molecular docking of benzophenone conjugated with oxadiazole sulphur bridge pyrazole pharmacophores as anti inflammatory and analgesic agents. Bioorg. Chem. 2019, 92, 103220. [CrossRef]

125. Karabanovich, G.; Němeček, J.; Valášková, L.; Carazo, A.; Konečná, K.; Stolaříková, J.; Hrabálek, A.; Pavliš, O.; Pávek, P.; Vávrová, K.; et al. S-substituted 3,5-dinitrophenyl 1,3,4-oxadiazole-2-thiols and tetrazole-5-thiols as highly efficient antitubercular agents. Eur. J. Med. Chem. 2017, 126, 369-383. [CrossRef]

126. Roh, J.; Karabanovich, G.; Vlčková, H.; Carazo, A.; Němeček, J.; Sychra, P.; Valášková, L.; Pavliš, O.; Stolaříková, J.; Klimešová, V.; et al. Development of water-soluble 3,5-dinitrophenyl tetrazole and oxadiazole antitubercular agents. Bioorg. Med. Chem. 2017, 25, 5468-5476. [CrossRef]

127. Channar, P.A.; Saeed, A.; Larik, F.A.; Rashid, S.; Iqbal, Q.; Rozi, M.; Younis, S.; Mahar, J. Design and synthesis of 2,6-di(substituted phenyl)thiazolo[3,2-b]-1,2,4-triazoles as $\alpha$-glucosidase and $\alpha$-amylase inhibitors, co-relative Pharmacokinetics and 3D QSAR and risk analysis. Biomed. Pharmacother. 2017, 94, 499-513. [CrossRef]

128. Shah, K.; Mujwar, S.; Gupta, J.K.; Shrivastava, S.K.; Mishra, P. Molecular Docking and In Silico Cogitation Validate Mefenamic Acid Prodrugs as Human Cyclooxygenase-2 Inhibitor. Assay Drug Dev. Technol. 2019, 17, 285-291. [CrossRef]

129. Morris, G.M.; Huey, R.; Lindstrom, W.; Sanner, M.F.; Belew, R.K.; Goodsell, D.S.; Olson, A.J. AutoDock4 and AutoDockTools4: Automated docking with selective receptor flexibility. J. Comput. Chem. 2009, 30, 2785-2791. [CrossRef]

130. Shimamura, T.; Sumikura, Y.; Yamazaki, T.; Tada, A.; Kashiwagi, T.; Ishikawa, H.; Matsui, T.; Sugimoto, N.; Akiyama, H.; Ukeda, H. Applicability of the DPPH Assay for Evaluating the Antioxidant Capacity of Food Additives Inter-laboratory Evaluation Study. Anal. Sci. 2014, 30, 717-721. [CrossRef]

131. Abramovič, H.; Grobin, B.; PoklarUlrih, N.; Cigić, B. Relevance and Standardization of In Vitro Antioxidant Assays: ABTS, DPPH, and Folin-Ciocalteu. J. Chem. 2018, 2018, 4608405. [CrossRef]

132. Moualek, I.; IratniAiche, G.; MestarGuechaoui, N.; Lahcene, S.; Houali, K. Antioxidant and anti-inflammatory activities of Arbutus unedo aqueous extract. Asian Pac. J. Trop. Biomed. 2016, 6, 937-944. [CrossRef]

133. Bondet, V.; Brand-Williams, W.; Berset, C. Kinetics and Mechanisms of Antioxidant Activity using the DPPH.Free Radical Method. LWTFood Sci. Technol. 1997, 30, 609-615. [CrossRef]

134. Gamez, E.J.C.; Luyengi, L.; Lee, S.K.; Zhu, L.-F.; Zhou, B.-N.; Fong, H.H.S.; Pezzuto, J.M.; Kinghorn, A.D. Antioxidant Flavonoid Glycosides from Daphniphyllum calycinum. J. Nat. Prod. 1998, 61, 706-708. [CrossRef] [PubMed]

135. Kim, D.-O.; Lee, K.W.; Lee, H.J.; Lee, C.Y. Vitamin C Equivalent Antioxidant Capacity (VCEAC) of Phenolic Phytochemicals. J. Agric. Food Chem. 2002, 50, 3713-3717. [CrossRef]

136. Cheng, Z.; Moore, J.; Yu, L. High-Throughput Relative DPPH Radical Scavenging Capacity Assay. J. Agric. Food Chem. 2006, 54, 7429-7436. [CrossRef]

137. Abdel-Hafez, E.-S.M.N.; Abuo-Rahma, G.E.-D.A.A.; Abdel-Aziz, M.; Radwan, M.F.; Farag, H.H. Design, synthesis and biological investigation of certain pyrazole-3-carboxylic acid derivatives as novel carriers for nitric oxide. Bioorg. Med. Chem. 2009, 17, 3829-3837. [CrossRef]

138. Aziz, H.A.; Moustafa, G.A.I.; Abbas, S.H.; Derayea, S.M.; Abuo-Rahma, G.E.-D.A.A. New norfloxacin/nitric oxide donor hybrids: Synthesis and nitric oxide release measurement using a modified Griess colorimetric method. Eur. J. Chem. 2017, 8, 119-124. [CrossRef]

139. Druckerei, C.H. European Pharmacopoeia, 10th Edition. Natrii Nitris, Nördlingen, Germany. 2020, pp. 3257-3258. Available online: https:/ / www.edqm.eu/en/news/ph-eur-10th-edition-now-available (accessed on 6 February 2021). 